Theo J.H.M. Eggen and Bernard P. Veldkamp (Editors)

\title{
Psychometrics in Practice at RCEC
}

RCEC 
ISBN 978-90-365-3374-4

DOI http://dx.doi.org/10.3990/3.9789036533744

Copyright (C) RCEC, Cito/University of Twente, Enschede, Netherlands, 2012.

All rights reserved. No part of this book may be reproduced, stored in a retrieval system, or transmitted, in any form or by any means, electronically, mechanically by photocopy, by recording, or otherwise, without prior written permission of the editors.

Print: Ipskamp Drukkers, Enschede. 


\section{Preface}

Education is of paramount importance in the lives of many children and young adults. It provides them with the necessary knowledge, skills and competences to participate in society. Besides, since lifelong learning is advocated as a necessary condition to excel at within the knowledge economy, it affects all of us. In the educational systems of the Netherlands examinations play an important role. During the educational process decisions are being made based on the results of assessment procedures, and examinations evaluate the performance of individuals, the performance of schools, the quality of educational programs, and allow entrance to higher levels of education. The future of individuals is often determined by measurement of competences in theoretical or practical tests and exams.

Educational measurement has its scientific roots in psychometrics. Psychometrics is an applied science serving developers and users of tests with methods that enable them to judge and to enhance the quality of assessment procedures. It focuses on the construction of instruments and procedures for measurement and deals with more fundamental issues related to the development of theoretical approaches to measurement. Solid research is needed to provide a knowledge base for examination and certification in the Netherlands.

For that reason the Research Center for Examinations and Certification (RCEC) was founded. RCEC, a collaboration of Cito and the University of Twente, was founded in 2008. Since its inception, RCEC has conducted a number of research projects often in cooperation with partners from the educational field, both from the Netherlands and abroad. One of the RCEC's main goals is to conduct scientific research on applied psychometrics. The RCEC is a research center for questions dealing with examinations and certification in education. The intention is that the results of its research should contribute to the improvement of the quality of examination procedures in the Netherlands and abroad.

This book is especially written for Piet Sanders, the founding father and first director of RCEC on the occasion of his retirement from Cito. All contributors to this volume worked with him on various projects. We admire his enthusiasm and knowledge of the field of educational measurement. It is in honor of him that we show some of the current results of his initiative. 
A broad range of topics is dealt with in this volume: from combining the psychometric generalizability and item response theories to the ideas for an integrated formative use of data-driven decision making, assessment for learning and diagnostic testing. A number of chapters pay attention to computerized (adaptive) and classification testing. Other chapters treat the quality of testing in a general sense, but for topics like maintaining standards or the testing of writing ability, the quality of testing is dealt with more specifically.

All authors are connected to RCEC as researchers. They present one of their current research topics and provide some insight into the focus of RCEC. The selection of the topics and the editing intends that the book should be of special interest to educational researchers, psychometricians and practitioners in educational assessment.

Finally, we want to acknowledge the support of Cito and the University of Twente for the opportunity they gave for doing our job. But most of all, we are grateful to the authors of the chapters who gave their valuable time for creating the content of the chapters, and to Birgit Olthof who took care of all the layout problems encountered in finishing the book.

Arnhem, Enschede, Princeton, May 2012.

Theo J.H.M. Eggen

Bernard P. Veldkamp 


\section{Contributors}

Cees A. W. Glas, University of Twente, Enschede, Netherlands, c.a.w.glas@utwente.nl

Theo J.H.M. Eggen, Cito, Arnhem / University of Twente, Netherlands, theo.eggen@ cito.nl

Anton Béguin, Cito, Arnhem, Netherlands, anton.beguin@ cito.nl

Bernard P. Veldkamp, University of Twente, Enschede, Netherlands, b.p.veldkamp@utwente.nl

Qiwei He, University of Twente, Enschede, Netherlands, q.he@utwente.nl

Muirne C.S. Paap, University of Twente, Enschede, Netherlands, m.c.s.paap@utwente.nl

Hiske Feenstra, Cito, Arnhem, Netherlands, hiske.feenstra@ cito.nl

Maarten Marsman. Cito, Arnhem, Netherlands, maarten.marsman@ cito.nl

Gunter Maris, Cito, Arnhem / University of Amsterdam, Netherlands, gunter.maris@ cito.nl

Timo Bechger, Cito, Arnhem, Netherlands, timo.bechger@ cito.nl

Saskia Wools, Cito, Arnhem, Netherlands, saskia.wools@ @ito.nl

Marianne Hubregtse, KCH, Ede, Netherlands, m.hubregtse@ kch.nl

Maaike M. van Groen, Cito, Arnhem, Netherlands, maaike.vangroen@cito.nl

Sebastiaan de Klerk, ECABO, Amersfoort, Netherlands, s.dklerk@ecabo.nl

Jorine A. Vermeulen, University of Twente, Enschede, Netherlands, jorine.vermeulen@ cito.nl

Fabienne M. van der Kleij, Cito, Arnhem, Netherlands, fabienne.vanderkleij@ cito.nl 


\section{Contents}

1 Generalizability Theory and Item Response Theory

Cees A.W. Glas

DOI http://dx.doi.org/10.3990/3.9789036533744.ch1

2 Computerized Adaptive Testing Item Selection in Computerized Adaptive Learning Systems

Theo J.H.M. Eggen

DOI http://dx.doi.org/10.3990/3.9789036533744.ch2

3 Use of Different Sources of Information in Maintaining Standards:

Examples from the Netherlands

Anton Béguin

DOI http://dx.doi.org/10.3990/3.9789036533744.ch3

$4 \quad$ Ensuring the Future of Computerized Adaptive Testing

Bernard P. Veldkamp

DOI http://dx.doi.org/10.3990/3.9789036533744.ch4

5 Classifying Unstructured Textual Data Using the Product Score Model:

An Alternative Text Mining Algorithm

Qiwei He and Bernard P. Veldkamp

DOI http://dx.doi.org/10.3990/3.9789036533744.ch5

6 Minimizing the Testlet Effect: Identifying Critical Testlet Features by

Means of Tree-Based Regression

Muirne C.S. Paap and Bernard P. Veldkamp

DOI : http://dx.doi.org/10.3990/3.9789036533744.ch6 
7 Mixed Methods: Using a Combination of Techniques to Assess

Writing Ability

Hiske Feenstra

DOI http://dx.doi.org/10.3990/3.9789036533744.ch7

8 Don't Tie Yourself to an Onion: Don't Tie Yourself to Assumptions of Normality

Maarten Marsman, Gunter Maris and Timo Bechger

DOI http://dx.doi.org/10.3990/3.9789036533744.ch8

9 Towards a Comprehensive Evaluation System for the Quality of Tests and Assessments

Saskia Wools

DOI http://dx.doi.org/10.3990/3.9789036533744.ch9

10 Influences on Classification Accuracy of Exam Sets: An Example from Vocational Education and Training

Marianne Hubregtse and Theo J.H.M. Eggen

DOI http://dx.doi.org/10.3990/3.9789036533744.ch10

11 Computerized Classification Testing and Its Relationship to

the Testing Goal

Maaike M. van Groen

DOI http://dx.doi.org/10.3990/3.9789036533744.ch11

12 An Overview of Innovative Computer-Based Testing

Sebastiaan de Klerk

DOI http://dx.doi.org/10.3990/3.9789036533744.ch12

13 Towards an Integrative Formative Approach of Data-Driven Decision

Making, Assessment for Learning, and Diagnostic Testing

Jorine A. Vermeulen and Fabienne M. van der Kleij

DOI http://dx.doi.org/10.3990/3.9789036533744.ch13 



\title{
Chapter 1
}

\section{Generalizability Theory and Item Response Theory}

\author{
Cees A.W. Glas
}

\begin{abstract}
Item response theory is usually applied to items with a selected-response format, such as multiple choice items, whereas generalizability theory is usually applied to constructed-response tasks assessed by raters. However, in many situations, raters may use rating scales consisting of items with a selected-response format. This chapter presents a short overview of how item response theory and generalizability theory were integrated to model such assessments. Further, the precision of the estimates of the variance components of a generalizability theory model in combination with two- and three-parameter models is assessed in a small simulation study.
\end{abstract}

Keywords: Bayesian estimation, item response theory, generalizability theory, Markov chain Monte Carlo

\section{Introduction}

I first encountered Piet Sanders when I started working at Cito in 1982. Piet and I came from different psychometric worlds: He followed generalizability theory (GT), whereas I followed item response theory (IRT). Whereas I spoke with reverence about Gerhard Fischer and Darrell Bock, he spoke with the same reverence about Robert Brennan and Jean Cardinet. Through the years, Piet invited all of them to Cito, and I had the chance to meet them in person. With a slightly wicked laugh, Piet told me the amusing story that Robert Brennan once took him aside to state, "Piet, I have never seen an IRT model work." Later, IRT played an important role in the book Test Equating, Scaling, and Linking by Kolen and Brennan (2004). Piet's and my views converged over time. His doctoral thesis "The Optimization of Decision Studies in Generalizability Theory" (Sanders, 1992) shows that he was clearly inspired by optimization approaches to test construction from IRT.

On January 14 and 15, 2008, I attended a conference in Neuchâtel, Switzerland, in honor of the 80th birthday of Jean Cardinet, the main European theorist of GT. My presentation was called "The Impact of Item Response Theory in Educational Assessment:

A Practical Point of View" and was later published in Mesure et Evaluation en Education (Glas, 2008). I remember Jean Cardinet as a very friendly and civilized gentleman. But he had a mission: 
It soon became clear that he wanted to show the psychometric world that GT was the better way and far superior to modernisms such as IRT. I adapted my presentation to show that there was no principled conflict between GT and IRT, and that they could, in fact, be combined. Jean seemed convinced. Below, I describe how IRT and GT can be combined. But first I shall present some earlier attempts of analyzing rating data with IRT.

\section{Some History}

Although in hindsight the combination of IRT and GT seems straightforward, creating the combination took some time and effort. The first move in that direction, made by Linacre $(1989,1999)$, was not very convincing. Linacre considered dichotomous item scores given by raters. Let $Y_{n r i}$ be an item score given by a rater $r\left(r=1, \ldots, N_{r}\right)$ on an item $i(i=1, \ldots, K)$ when assessing student $n(n=1, \ldots, N) . Y_{n r i}$ is equal to 0 or 1 . Define the logistic function $\Psi($.$) as$

$$
\Psi(\tau)=\frac{\exp (\tau)}{1+\exp (\tau)}
$$

Conditional on a person ability parameter $\theta_{n}$, the probability of a positive item score is defined as $\operatorname{Pr}\left(Y_{n r i}=1 \mid \theta_{n}\right)=P_{n r i}=\Psi\left(\tau_{n r i}\right)$, with

$$
\tau_{n r i}=\theta_{n}-\beta_{i}+\rho_{r}
$$

where $\beta_{i}$ is an item parameter and $\rho_{r}$ is a rater effect. The model was presented as a straightforward generalization of the Rasch model (Rasch, 1960); in fact, it was seen as a straightforward application of the linear logistic test model (LLTM) (Fischer, 1983). That is, the probability of the scores given to a respondent was given by

$$
\prod_{i} \prod_{r} P_{n r i}^{y_{n r i}}\left(1-P_{n r i}\right)^{1-y_{n r i}}
$$

In my $\mathrm{PhD}$ thesis, I argued that this is a misspecification, because the assumption of local independence made here is violated: The responses of the different raters are dependent because they depend on the response of the student (Glas, 1989). 
Patz and Junker (1999) criticize Linacre's approach on another ground: LLTMs require that all items have a common slope or discrimination parameter; therefore, they suggest using the logistic model given in Equation (1) with the argument

$$
\tau_{n r i}=\alpha_{i} \theta_{n}-\beta_{i}+\rho_{r i}
$$

where $\alpha_{i}$ is a discrimination parameter and $\rho_{r i}$ stands for the interaction between an item and a rater. However, this does not solve the dependence between raters. Therefore, we consider the following alternative. The discrimination parameter is dropped for convenience; the generalization to a model with discrimination parameters is straightforward. Further, we assume that the students are given tasks indexed $t\left(t=1, \ldots, N_{t}\right)$, and the items are nested within the tasks. A generalization to a situation where tasks and items are crossed is straightforward. Further, item $i$ pertains to task $t(i)$. Consider the model given in Equation (1) with the argument

$$
\tau_{n r t i}=\theta_{n}+\delta_{n t(i)}-\beta_{i}+\rho_{r}
$$

The parameter $\delta_{n t(i)}$ models the interaction between a student and a task. Further, Patz and Junker (1999) define $\theta_{n}$ and $\delta_{n t(i)}$ as random effects, that is, they are assumed to be drawn from some distribution (i.e., the normal distribution). The parameters $\beta_{i}$ and $\rho_{r}$ may be either fixed or random effects.

To assess the dependency structure implied by this model, assume $\tau_{n r t i}$ could be directly observed. For two raters, say $r$ and $s$, scoring the same item $i$, it holds that $\operatorname{Cov}\left(\tau_{n r t i}, \tau_{n s t i}\right)=\operatorname{Cov}\left(\theta_{n}, \theta_{n}\right)+\operatorname{Cov}\left(\delta_{n t(i)}, \delta_{n t(i)}\right)=\sigma_{n}^{2}+\sigma_{n t}^{2}$. This also holds for two items related to the same task. If two items, say $i$ and $j$, are related to the same task, that is, if $t(i)=t(j)=t$, then $\operatorname{Cov}\left(\tau_{n r t i}, \tau_{n s t j}\right)=\operatorname{Cov}\left(\theta_{n}, \theta_{n}\right)+\operatorname{Cov}\left(\delta_{n t(i)}, \delta_{n t(j)}\right)=\sigma_{n}^{2}+\sigma_{n t}^{2}$.

If items are related to different tasks, that is, if $t(i) \neq t(j)$, then $\operatorname{Cov}\left(\tau_{n r t i}, \tau_{n s t j}\right)=\sigma^{2}$. So, $\sigma_{n t}^{2}$ models the dependence of item responses within a task. 


\section{Combining IRT and GT}

The generalization of this model to a full-fledged generalizability model is achieved through the introduction of random main effects for tasks $\pi_{t}$, random effects for the interaction between students and raters $\gamma_{n r}$, and students and tasks $\eta_{t r}$. The model then becomes the logistic model in Equation (1) with the argument

$$
\tau_{n r t i}=\theta_{n}-\beta_{i}+\rho_{r}+\pi_{t}+\delta_{n t(i)}+\gamma_{n r}+\eta_{t r}
$$

The model can be conceptualized by factoring it into a measurement model and structural model, that is, into an IRT measurement model and a structural random effects analysis of variance (ANOVA) model. Consider the likelihood function

$$
\prod_{i} \prod_{r} P_{n r i}^{y_{n i}}\left(\tilde{\tau}_{n r t(i)}\right)\left(1-P_{n r i}\left(\tilde{\tau}_{n r t(i)}\right)\right)^{1-y_{n r i}} N\left(\tilde{\tau}_{n r t(i)}\right)
$$

where

$$
\tilde{\tau}_{n r t}=\theta_{n i}+\rho_{r}+\pi_{t}+\delta_{n t}+\gamma_{n r}+\eta_{t r}
$$

is a sum of random effects, $P_{n r i}\left(\tilde{\tau}_{n r t(i)}\right)$ is the probability of a correct response given $\tilde{\tau}_{n r t}$ and the item parameter $\beta_{i}$, and $N\left(\tilde{\tau}_{n r t(i)}\right)$ is the density of $\tilde{\tau}_{n r t}$, which is assumed to be a normal density. If the distribution of $\tilde{\tau}_{n r t}$ is normal, the model given in Equation (2) is completely analogous to the GT model, which is a standard ANOVA model.

This combination of IRT measurement model and structural ANOVA model was introduced by Zwinderman (1991) and worked out further by Fox and Glas (2001). The explicit link with GT was made by Briggs and Wilson (2007).

They use the Rasch model as a measurement model and the GT model - that is, an ANOVA model - as the structural model. The structural model implies a variance decomposition

$$
\sigma_{\tau}^{2}=\sigma_{n}^{2}+\sigma_{t}^{2}+\sigma_{r}^{2}+\sigma_{n t}^{2}+\sigma_{n r}^{2}+\sigma_{t r}^{2}+\sigma_{e}^{2},
$$


and these variance components can be used to construct the well-known agreement and reliability indices as shown in Table 1.

Table 1 Indices for Agreement and Reliability for Random and Fixed Tasks Type of Assessment Index

Random tasks, agreement

$$
\frac{\sigma_{n}^{2}}{\sigma_{n}^{2}+\sigma_{t}^{2} / N_{t}+\sigma_{r}^{2} / N_{r}+\sigma_{n t}^{2} / N_{t}+\sigma_{n r}^{2} / N_{r}+\sigma_{t r}^{2} / N_{r} N_{t}+\sigma_{e}^{2} / N_{r} N_{t}}
$$

Random tasks, reliability

$$
\frac{\sigma_{n}^{2}}{\sigma_{n}^{2}+\sigma_{n t}^{2} / N_{t}+\sigma_{n r}^{2} / N_{r}+\sigma_{t r}^{2} / N_{r} N_{t}+\sigma_{e}^{2} / N_{r} N_{t}}
$$

Fixed tasks, agreement

$$
\frac{\sigma_{n}^{2}+\sigma_{n t}^{2}}{\sigma_{n}^{2}+\sigma_{n t}^{2}+\sigma_{r}^{2} / N_{r}+\sigma_{n r}^{2} / N_{r}+\sigma_{t r}^{2} / N_{r}+\sigma_{e}^{2} / N_{r}}
$$

Fixed tasks, reliability

$$
\frac{\sigma_{n}^{2}+\sigma_{n t}^{2}}{\sigma_{n}^{2}+\sigma_{n t}^{2}+\sigma_{r}^{2} / N_{r}+\sigma_{e}^{2} / N_{r}}
$$

Note: $N_{t}=$ number of tasks; $N_{r}=$ number of raters.

\section{Parameter Estimation}

The model considered here seems quite complicated; however, conceptually, estimation in a Bayesian framework using Markov chain Monte Carlo (MCMC) computational methods is quite straightforward. The objective of the MCMC algorithm is to produce samples of the parameters from their posterior distribution. Fox and Glas (2001) developed a Gibbs sampling approach, which is a generalization of a procedure for estimation of the two-parameter normal ogive (2PNO) model by Albert (1992). For a generalization of the three-parameter normal ogive (3PNO) model, refer to Béguin and Glas (2001). Below, it will become clear that to apply this approach, we first need to reformulate the model from a logistic representation to a normal-ogive representation. That is, we assume that the conditional probability of a positive item score is defined as $\operatorname{Pr}\left(Y_{n r t i}=1 \mid \tau_{n r t i}\right)=P_{n r t i}=\Phi\left(\tau_{n r t i}\right)$, where $\Phi($.$) is the cumulative$ normal distribution, i.e.,

$$
\Phi(\tau)=(2 \pi)^{-1 / 2} \int_{-\infty}^{\tau} \exp \left(-t^{2} / 2\right) d t
$$

In the 3PNO model, the probability of a positive response is given by

$$
P_{n r t i}=\gamma_{i}+\left(1-\gamma_{i}\right) \Phi\left(\tau_{n r t i}\right)
$$


where $\gamma_{i}$ is a guessing parameter.

Essential to Albert's approach is a data augmentation step (Tanner \& Wong, 1987), which maps the discrete responses to continuous responses. Given these continuous responses, the posterior distributions of all other parameters become the distributions of standard regression models, which are easy to sample from. We outline the procedure for the 2PNO model. We augment the observed data $Y_{n r t i}$ with latent data $Z_{n r t i}$, where $Z_{n r t i}$ is a truncated normally distributed variable, i.e.,

$$
Z_{n r t i} \mid Y_{n r t i} \sim \begin{cases}N\left(\tau_{n r t i}, 1\right) \text { truncated at the left by } 0 & \text { if } Y_{n r t i}=1 \\ N\left(\tau_{n r t i}, 1\right) \text { truncated at the right by } 0 & \text { if } Y_{n r t i}=0 .\end{cases}
$$

Note that this data augmentation approach is based on the normal-ogive representation of the IRT model, which entails the probability of a positive response is equal to the probability mass left from the cut-off point $\tau_{n r t i}$.

Gibbs sampling is an iterative process, where the parameters are divided into a number of subsets, and a random draw of the parameters in each subset is made from its posterior distribution given the random draws of all other subsets. This process is iterated until convergence. In the present case, the augmented data $Z_{n r t i}$ are drawn given starting values of all other parameters using Equation (3). Then the item parameters are drawn using the regression model $Z_{n r t i}=\tilde{\tau}_{n r t}-\beta_{i}+\varepsilon_{n t r i}$, with $\quad \tilde{\tau}_{n r t}=\theta_{n}+\rho_{r}+\pi_{t}+\delta_{n t}+\gamma_{n r}+\eta_{t r} \quad$ where all parameters except $\beta_{i}$ have normal priors. If discrimination parameters are included, the regression model becomes $Z_{n r t i}=\alpha_{i} \tilde{\tau}_{n r t}-\beta_{i}+\varepsilon_{n t r i}$.

The priors for $\beta_{i}$ can be either normal or uninformative, and the priors for $\alpha_{i}$ can be normal, lognormal, or confined to the positive real numbers. Next, the other parameters are estimated using the standard ANOVA model $Z_{n r t i}-\beta_{i}=\theta_{n}+\rho_{r}+\pi_{t}+\delta_{n t}+\gamma_{n r}+\eta_{t r}+\varepsilon_{n r t i}$. These steps are iterated until the posterior distributions stabilize. 


\section{A Small Simulation Study}

The last section pertains to a small simulation to compare the use of the $2 \mathrm{PNO}$ model with the use of the 3PNO model. The simulation is related to the so-called bias-variance trade-off. When estimating the parameters of a statistical model, the mean-squared error (i.e., the mean of the squared difference between the true value and the estimates over replications of the estimation procedure) is the sum of two components: the squared bias and the sampling variance (i.e., the squared standard error). The bias-variance trade-off pertains to the fact that, on one hand, more elaborated models with more parameters tend to reduce the bias, whereas on the other hand, adding parameters leads to increased standard errors. At some point, using a better fitting, more precise model may be counterproductive because of the increased uncertainty reflected in large standard errors. That is, at some point, there are not enough data to support a too elaborate model.

In this simulation, the 3PNO model is the elaborate model, which may be true but hard to estimate, and the $2 \mathrm{PNO}$ model is an approximation, which is beside the truth but easier to estimate. The data were simulated as follows. Sample sizes of 1,000 and 2,000 students were used. Each simulation was replicated 100 times. The test consisted of five tasks rated by two raters both scoring five items per task. Therefore, the total number of item responses was 50, or 25 for each of the two raters. The responses were generated using the 3PNO model. For each replication, the item location parameters $\beta_{i}$ were drawn from a standard normal distribution, the item discrimination parameters $\alpha_{i}$ were drawn from a normal distribution with a mean equal to 1.0 and a standard deviation equal to 0.25 , and the guessing parameters $\gamma_{i}$ were drawn from a beta distribution with parameters 5 and 20. The latter values imply an average guessing parameter equal to 0.25 . These distributions were also used as priors in the estimation procedure.

The used variance components are shown in the first column of Table 2. The following columns give estimates of the standard error and bias obtained over the 100 replications, using the two sample sizes and the 2PNO and 3PNO models, respectively.

In every replication, the estimates of the item parameters and the variance components were obtained using the Bayesian estimation procedure by Fox and Glas (2001) and Béguin and Glas (2001), outlined above. The posterior expectation (EAP) was used as a point estimate. Besides a number of variance components, the reliability $\rho^{2}$ for an assessment with random tasks was estimated. The bias and standard errors for the reliability are given in the last row of Table 2 . 
Note that, overall, the standard errors of the EAPs obtained using the $2 \mathrm{PNO}$ model are smaller than the standard errors obtained using the 3PNO model. On the other hand, the bias for the 2PNO model is generally larger. These results are in accordance with the author's expectations.

Table 2 Comparing Variance Component Estimates for 2PNO and 3PNO Models

\begin{tabular}{llllllllll}
\hline Variance & \multicolumn{9}{c}{$\mathrm{N}=1,000$} \\
Components/ & True & 2PNO & & 3PNO & & 2PNO & & 3PNO \\
Reliability & Values & & & & & & & & \\
Coefficient & & & & & & & & & \\
\hline & & SE & Bias & SE & Bias & SE & Bias & SE & Bias \\
\hline$\hat{\sigma}_{n}^{2}$ & 1.0 & .0032 & .0032 & .0036 & .0028 & .0021 & .0024 & .0028 & .0009 \\
$\hat{\sigma}_{n t}^{2}$ & 0.2 & .0027 & .0024 & .0033 & .0022 & .0023 & .0021 & .0021 & .0010 \\
$\hat{\sigma}_{n r}^{2}$ & 0.2 & .0043 & .0039 & .0054 & .0036 & .0022 & .0036 & .0043 & .0027 \\
$\hat{\sigma}_{t r}^{2}$ & 0.2 & .0056 & .0041 & .0066 & .0033 & .0036 & .0047 & .0046 & .0039 \\
$\hat{\sigma}_{\varepsilon}^{2}$ & 0.2 & .0047 & .0015 & .0046 & .0014 & .0028 & .0012 & .0037 & .0012 \\
$\rho^{2}$ & 0.85 & .0396 & .0105 & .0401 & .0106 & .0254 & .0101 & .0286 & .0104
\end{tabular}

Note: $2 \mathrm{PNO}=$ two-parameter normal ogive; $3 \mathrm{PNO}=$ three-parameter normal ogive; $\mathrm{SE}=$ standard error

\section{Conclusion}

This chapter showed that psychometricians required some time and effort to come up with a proper method for analyzing rating data using IRT. Essential to the solution was the distinction between a measurement model (i.e., IRT) and a structural model (i.e., latent linear regression model). The parameters of the combined measurement and structural models can be estimated in a Bayesian framework using MCMC computational methods.

In this approach, the discrete responses are mapped to continuous latent variables, which serve as the dependent variables in a linear regression model with normally distributed components. This chapter outlined the procedure for dichotomous responses in combination with the 2PNO model, but generalizations to the $3 \mathrm{PNO}$ model and to models for polytomous responses - e.g., the partial credit model (Masters, 1982), the generalized partial credit model (Muraki, 1992), the graded response model (Samejima, 1969), and the sequential model (Tutz, 1990) — are readily available (see, for instance, Johnson \& Albert, 1999). 
However, nowadays, developing specialized software for combinations of IRT measurement models and structural models is no longer strictly necessary. Many applications can be created in WinBUGS (Spiegelhalter, Thomas, Best, \& Lunn, 2004). Briggs and Wilson (2007) give a complete WinBUGS script to estimate the GT model in combination with the Rasch model. Although WinBUGS is a valuable tool for the advanced practitioner, it also has a drawback that is often easily overlooked: It is general-purpose software, and the possibilities for evaluation of model fit are limited.

Regardless, the present chapter may illustrate that important advances in modeling data from rating have been made over the past decade, and the combined IRT and GT model is now just another member of the ever-growing family of latent variable models (for a nice family picture, see, for instance, Skrondal \& Rabe-Hesketh, 2004).

\section{References}

Albert, J. H. (1992). Bayesian estimation of normal ogive item response functions using Gibbs sampling. Journal of Educational Statistics, 17, 251-269.

Béguin, A. A., \& Glas, C. A. W. (2001). MCMC estimation and some model-fit analysis of multidimensional IRT models. Psychometrika, 66, 541-562.

Briggs, D.C., \& Wilson, M. (2007). Generalizability in item response modeling. Journal of Educational Measurement, 44, 131-155.

Fischer, G. H. (1983). Logistic latent trait models with linear constraints. Psychometrika, 48, 3-26.

Fox, J. P., \& Glas, C. A. W. (2001). Bayesian estimation of a multilevel IRT model using Gibbs sampling. Psychometrika, 66, 271-288.

Glas, C. A. W. (1989). Contributions to estimating and testing Rasch models. Unpublished $\mathrm{PhD}$ thesis, Enschede, University of Twente.

Glas, C. A. W. (2008). Item response theory in educational assessment and evaluation. Mesure et Evaluation en Education, 31, 19-34.

Johnson, V. E., \& Albert, J. H. (1999). Ordinal data modeling. New York: Springer.

Kolen, M. J., \& Brennan, R. L. (2004). Test equating, scaling, and linking: Methods and practices (2nd ed.). New York: Springer.

Linacre, J. M. (1989). Many-facet Rasch measurement. Chicago: MESA Press.

Linacre, J. M. (1999). FACETS (Version 3.17) [Computer software]. Chicago: MESA Press. Masters, G. N. (1982). A Rasch model for partial credit scoring. Psychometrika, 47, 149-174. 
Muraki, E. (1992). A generalized partial credit model: Application of an EM algorithm. Applied Psychological Measurement, 16, 159-176.

Patz, R. J., \& Junker, B. (1999). Applications and extensions of MCMC in IRT: Multiple item types, missing data, and rated responses. Journal of Educational and Behavioral Statistics, 24, 342-366.

Rasch, G. (1960). Probabilistic models for some intelligence and attainment tests. Copenhagen: Danish Institute for Educational Research.

Samejima, F. (1969). Estimation of latent ability using a pattern of graded scores. Psychometrika, Monograph Supplement, No. 17.

Sanders, P. F. (1992). The optimization of decision studies in generalizability theory. Doctoral thesis, University of Amsterdam.

Skrondal, A., \& Rabe-Hesketh, S. (2004). Generalized latent variable modeling: Multilevel, longitudinal, and structural equation models. Boca Raton, FL: Chapman \& Hall/CRC.

Spiegelhalter, D., Thomas, A., Best, N., \& Lunn, D. (2004). WinBUGS 1.4. Retrieved from http://www.mrc-bsu.cam.ac.uk/bugs

Tanner, M. A., \& Wong, W. H. (1987). The calculation of posterior distributions by data augmentation [with discussion]. Journal of the American Statistical Association, 82, 528-540.

Tutz, G. (1990). Sequential item response models with an ordered response. British Journal of Mathematical and Statistical Psychology, 43, 39-55.

Zwinderman, A. H. (1991). A generalized Rasch model for manifest predictors. Psychometrika, 56, 589-600. 


\title{
Chapter 2
}

\section{Computerized Adaptive Testing Item Selection in Computerized Adaptive Learning Systems}

\author{
Theo J.H.M. Eggen
}

\begin{abstract}
Item selection methods traditionally developed for computerized adaptive testing (CAT) are explored for their usefulness in item-based computerized adaptive learning (CAL) systems. While in CAT Fisher information-based selection is optimal, for recovering learning populations in CAL systems item selection based on Kullback-Leibner information is an alternative.
\end{abstract}

Keywords: Computer-based learning, computerized adaptive testing, item selection

\section{Introduction}

In the last few decades, many computerized learning systems have been developed. For an overview of these systems and their main characteristics, see Wauters, Desmet and Van den Noortgate (2010). In so-called intelligent tutoring systems (Brusilovsky, 1999), the learning material is presented by learning tasks or items, which are to be solved by the learner. In some of these systems, not only the content of the learning tasks but also the difficulty can be adapted to the needs of the learner. The main goal in such a computerized adaptive learning (CAL) system is to optimize the student's learning process. An example of such an itembased CAL system is Franel (Desmet, 2006), a system developed for learning Dutch and French. If in item-based learning systems feedback or hints are presented to the learner, the systems can also be considered testing systems in which the main goal of testing is to support the learning process, known as assessment for learning (William, 2011). With this, a link is made between computerized learning systems and computerized testing systems.

Computerized testing systems have many successful applications. Computerized adaptive testing (CAT) is based on the application of item response theory (IRT). (Wainer, 2000; Van der Linden \& Glas, 2010). In CAT, for every test-taker a different test is administered by selecting items from an item bank tailored to the ability of the test taker as demonstrated by the responses given thus far. So, in principle, each test-taker is administered a different test whose composition is optimized for the person. 
The main result is that in CAT the measurement efficiency is optimized. It has been shown several times that CAT need fewer items, only about $60 \%$, to measure the test-taker's ability with the same precision. CAT and item-based CAL systems have several similarities: in both procedures, items are presented to persons dependent on earlier outcomes, using a computerized item selection procedure. However, the systems differ because CAT is based on psychometric models from IRT, while CAL is based on learning theory. In addition, the main goal in CAT systems is optimal measurement efficiency and in CAL systems optimal learning efficiency. Nevertheless, applying IRT and CAT in item-based CAL systems can be very useful. However, a number of problems prevent the application of a standard CAT approach in CAL systems. One important unresolved point is the item selection in such systems. In this chapter, traditional item selection procedures used in CAT will be evaluated in the context of using them in CAL systems. An alternative selection procedure, developed for better fit to the goal in CAL systems, is presented and compared to the traditional ones.

\section{Item Selection in CAT}

The CAT systems considered in this chapter presupposes the availability of an IRT calibrated item bank. The IRT model used is the two-parameter logistic model (2PL) (Birmbaum, 1968):

$$
p_{i}(\theta)=\mathrm{P}\left(X_{i}=1 \mid \theta\right)=\frac{\exp \left(a_{i}\left(\theta-b_{i}\right)\right)}{1+\exp \left(a_{i}\left(\theta-b_{i}\right)\right)}
$$

in which a specification is given of the relation between the ability, $\theta$, of a person and the probability of correctly answering item $i, X_{i}=1 . b_{i}$ is the location or difficulty parameter, and $a_{i}$ the discrimination parameter.

In CAT, the likelihood function is used for estimating a student's ability. Given the scores on $k$ items $x_{i}, i=1, \ldots, k$ this function is given by

$$
L\left(\theta ; x_{1}, \ldots, x_{k}\right)=\prod_{i=1}^{k} p_{i}(\theta)^{x_{i}}\left(1-p_{i}(\theta)^{\left(1-x_{i}\right)}\right.
$$


In this chapter, a statistically sound estimation method, the value of $\theta$ maximizing a weighted likelihood function (Warm, 1989), is used. This estimate after $k$ items is given by:

$$
\hat{\theta}_{k}=\max _{\theta}\left(\sum_{i=1}^{k} \mathrm{I}_{i}(\theta)\right)^{1 / 2} L\left(\theta ; x_{1}, \ldots, x_{k}\right)
$$

In this expression, the likelihood $L\left(\theta ; x_{1}, \ldots, x_{k}\right)$ is weighted by another function of the ability, $\mathrm{I}_{i}(\theta)$. This function, the item information function, plays a major role in item selection in CAT. In CAT, after every administered item, a new item that best fits the estimated ability is selected from the item bank. The selection of an item is based on the Fisher information function, which is defined as $\mathrm{I}_{i}(\theta)=E\left(\left(\partial L\left(\theta ; x_{i}\right) / \partial \theta\right) / L\left(\theta ; x_{i}\right)\right)^{2}$. The item information function, a function of the ability $\theta$, expresses the contribution an item makes to the accuracy of the measurement of the student's ability. This is readily seen, when it is realized that the standard error of the ability estimate can be written in terms of the sum of the item information of all the administered items:

$$
\operatorname{se}\left(\hat{\theta}_{k}\right)=1 /\left(\sum_{i=1}^{k} I_{i}\left(\hat{\theta}_{k}\right)\right)^{1 / 2}
$$

The item with maximum information at the current ability estimate, $\hat{\boldsymbol{\theta}}_{\boldsymbol{k}}$, is selected in CAT. Because this selection method searches for each person the items on which he or she has a success probability of 0.50 , we will denote this method by FI50.

\section{Item Selection in CAL Systems}

Item selection methods in traditional CAT aim for precisely estimating ability; in CAL systems, however, optimizing the learning process, not measuring, is the main aim. Although learning can be defined in many ways, an obvious operationalization is to consider learning effective if the student shows growth in ability. In an item-based learning system, a student starts at a certain ability level, and the goal is that at the end his or her ability level is higher. The ultimate challenge is then to have an item selection method that advances learning as much as possible. 
The possible item selection method explored here is based on Kullback-Leibner (K-L) information. In K-L information-based item selection, the items that discriminate best between two ability levels are selected. Eggen (1999) showed that selecting based on K-L information is a successful alternative for Fisher information-based item selection when classification instead of ability estimation is the main testing goal.

$\mathrm{K}-\mathrm{L}$ information is in fact a distance measure between two probability distributions or, in this context, the distance between the likelihood functions on two points on the ability scale. Suppose we have for a person two ability estimates at two time points $\theta_{t 1}$ and $\theta_{t 2}$.

Then we can formulate the hypotheses that $\mathrm{H} 0: \theta_{t 1}=\theta_{t 2}$ against $\mathrm{H} 1: \theta_{t 1}<\theta_{t 2} . \mathrm{H} 0$ means that all observations are from the same distribution, and if $\mathrm{H} 1$ is true, this means that there is real improvement between the two estimates in time. The K-L distance between these hypotheses is given $k$ items with response $\underline{x}_{k}=\left(x_{1}, \ldots, x_{k}\right)$ have been administered is

$$
K\left(\hat{\theta}_{t 2} \| \hat{\theta}_{t 1}\right) \equiv E\left[\ln \frac{L\left(\theta_{t 2} ; \underline{x}_{k}\right)}{L\left(\hat{\theta}_{t 1} ; \underline{x}_{k}\right)}\right]=\sum_{i=1}^{k} E\left[\ln \frac{L\left(\theta_{t 2} ; \underline{x}_{i}\right)}{L\left(\hat{\theta}_{t 1} ; \underline{x}_{i}\right)}\right]=\sum_{i=1}^{k} K_{i}\left(\hat{\theta}_{t 2} \| \hat{\theta}_{t 1}\right) .
$$

If we now select items that maximize this K-L distance, we select the items that maximally contribute to the power of the test to distinguish between the two hypotheses: H0, the ability does not change, versus H1, growth in ability, or learning, has taken place.

In practice, there are several possibilities for selecting the two points between which the $\mathrm{K}-\mathrm{L}$ distance is maximized. In this chapter, we will study selection using the two ability estimates based on the first and the second half of the administered items. (See, Eggen, 2011, for other possibilities.) Thus, if the number of administered items is cl (the current test length), the next item selected is the one that has the largest the K-L distance at $\hat{\theta}_{t_{2}}\left(x_{c l / 2}, x_{1+c l / 2}, \ldots, x_{c l}\right)$ and $\hat{\theta}_{t_{1}}\left(x_{1}, x_{2}, \ldots, x_{c l / 2}\right)$. This selection method is denoted by K-Lmid.

Item selection methods based on the difficulty level of the items are often considered in CAL systems. Theories relate the difficulty of the items to the motivation of learners and possible establishing more efficient learning for students (Wauters, Desmet, \& Van den Noortgate, 2012). Thus, an alternative item selection method giving items with a high or low difficulty level will be studied. If we select in CAT items with maximum Fisher information at the current ability estimate, with a good item bank items will be selected for which a person has a success probability of 0.50 . Bergstrom. 
Lunz and Gershon (1992) and Eggen and Verschoor (2006) developed methods for selecting easier (or harder) items while at the same time maintaining the efficiency of estimating the ability as much as possible. In this chapter, we consider selecting harder items with a success probability of 0.35 at the current ability estimate. (We will label this method FI35.)

\section{Comparing the Item Selection Methods for Possible Use in CAL Systems}

In evaluating the usefulness of selection methods in CAL systems, simulation studies have been conducted. In these simulation studies, it is not possible to evaluate the item selection methods regarding whether the individuals' learning is optimized.

Instead, only the possibility of recovering learning is compared. If learning takes place during testing, the ability estimates should show that.

In the simulation studies reported, the item bank used consisted of 300 items following the 2PL model with $\beta \sim \mathrm{N}(0,0.35)$ and $\ln \alpha \sim N(1,0.3)$. Testing starts with one randomly selected item of intermediate difficulty and has a fixed test length of 40 items. In the simulation, samples of $j=1, \ldots ., N=100.000$ abilities were drawn from the normal distribution. Three different populations were considered representing different learning scenarios.

1. Fixed population: all simulees are from the same population and do not change during testing: $\theta \sim \mathrm{N}(0,0.35)$.

2. The population shows a step in growing in ability: in the first 20 items, $\theta \sim \mathrm{N}(0,0.35)$; after that, from item 21 to $40 \theta \sim \mathrm{N}(\delta, 0.35) . \delta>0$ represents the learning step that took place.

3. The population is growing linearly: $\theta$ is drawn from the normal distribution with increasing mean with the item position $\ell$ in the test: $\theta \sim \mathrm{N}(\ell . \delta / 40,0,0.35)$

To evaluate the performance of item selection methods in a CAT simulation, the root mean square error of the ability after administering $\ell$ items is commonly used:

$$
\operatorname{rmse} \theta(\ell)=\left(\sum_{j=1}^{N}\left(\theta_{j}^{\ell}-\hat{\theta}_{j}^{\ell}\right)^{2} / N\right)^{1 / 2}
$$

This is a useful criterion for evaluating the estimation accuracy of the ability when simulees with fixed abilities are considered. 
However, if we want to evaluate the recovery of a growing ability, a related measure, the root mean square of the difference in abilities $\operatorname{rmse} \delta(\ell)$, is more appropriate. If $\delta_{j}^{\ell}=\theta_{2 j}^{\ell}-\theta_{1 j}^{\ell}$ is the difference between the true abilities on two points in time and $\hat{\delta}_{j}^{\ell}=\hat{\theta}_{2 j}^{\ell}-\hat{\theta}_{1 j}^{\ell}$ is the difference between the estimated abilities on the two time points in time, this is given by

$$
\operatorname{rmse} \delta(\ell)=\left(\sum_{j=1}^{N}\left(\delta_{j}^{\ell}-\delta_{j}^{\ell}\right)^{2} / N\right)^{1 / 2}
$$

\section{Results}

The results for comparing the item selection methods in the fixed population at the full test length of 40 items is given in Table 1. This confirms what was expected. In CAT, selecting items with maximum information at the current ability is the most efficient. The difference with random item selection is huge, while we lose some efficiency when we select harder items. The item selection developed for the cases in which learning takes place hardly causes any loss in efficiency when the population is not increasing.

Table 1 rmse $\theta(\ell)$ at full test length for a fixed population

\begin{tabular}{ll}
\hline Selection & rmse $\theta(40)$ \\
\hline FI50 & 0.0972 \\
FI35 & 0.0989 \\
KL-MID & 0.0974 \\
Random & 0.1547 \\
\hline
\end{tabular}

If we consider in the fixed population the $r m s e \theta(\ell)$ as a function of the test length, then for all selection methods this is decreasing with the test length quickly approaching the maximum accuracy to be reached (in this example, about 0.09 at about 35 items). In Figure 1, rmse $\theta(\ell)$ is shown in a population that is growing linearly during testing. 


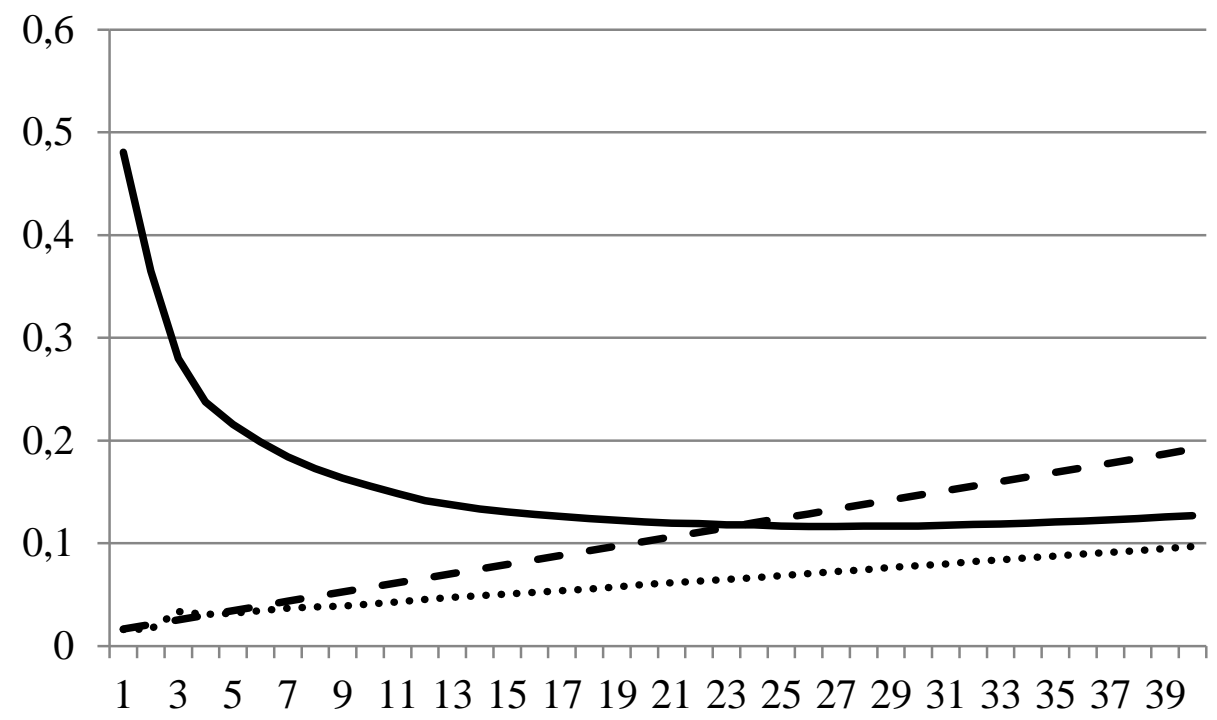

Figure 1 True ability, estimated ability and $r m s e \theta(\ell)$ in population growing linearly $\delta=0.175$

In Figure 1, the dashed line (---) gives the true (growing) abilities, and the points (...) are the estimated abilities; $r m s e \theta(\ell)$ is given by the solid line $(-)$. In this situation, where the estimated abilities always lag behind the development of true ability, the rmse $\theta(\ell)$ is first decreasing and later increasing with the test length. This illustrates that it cannot be a good criterion for judging the recovery of growth in ability.

Therefore, the selection methods are compared on the rmse $\delta(\ell)$. In all the simulation studies conducted, rmse $\delta(\ell)$ is monotone decreasing with growing test length. Thus, the results in Table 2 are given for the full test length of 40 items. The results refer to the situation in which the increase in ability during testing is 0.175 (0.5 SD [standard deviation] of the true ability distribution).

Table 2 rmse $\delta(\ell)$ for fixed, stepwise and linearly growing population

\section{Growth scenario}

\begin{tabular}{llll}
\hline Selection & Fixed & Step & Linear \\
FI50 & 0.192 & 0.196 & 0.195 \\
FI35 & 0.195 & 0.199 & 0.197 \\
KL-MID & 0.192 & 0.195 & 0.194 \\
Random & 0.303 & 0.306 & 0.304 \\
\hline
\end{tabular}


To recover growth in ability, the differences between the item selection methods show about the same pattern as reported on the measurement accuracy in a fixed population: random item selection performs badly, while selecting harder items also has a negative influence on the rmse $\delta(\ell)$. The differences between selecting with FI50 and the KL-mid method are small; however, in populations where there is growth in ability the selection method based on the KL information performs a bit better. Figure 2 shows where for which ability levels in the population with linear growth the small difference between the FI50 and KL-Mid method occurs.

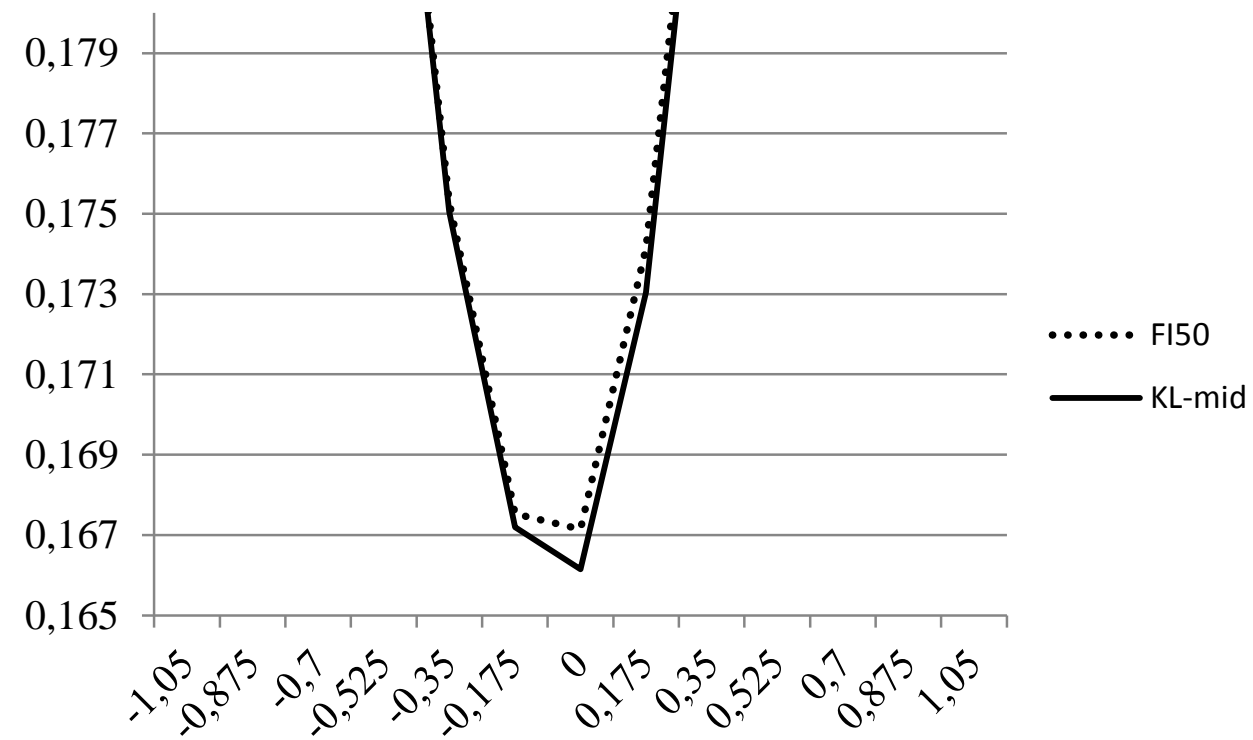

Figure $2 \operatorname{rmse} \delta(\ell)$ for $\ell=40$ for FI50 en KL-mid item selection as function of ability

Figure 2 shows there are only very small differences in performances for abilities around the mean of the population, which could be possibly be due to the item bank, which was constructed so that the distribution of difficulties is centered on the population mean of 0 . Differences between the item selection method may appear only when there are many items of the appropriate difficulty available.

\section{Discussion}

In computerized adaptive testing, item selection with maximum Fisher information at the ability estimate determined during testing based on the given response is most efficient for measuring individuals' abilities. 
In this chapter, a K-L information-based item selection procedure was proposed for adaptively selecting items in a computerized adaptive learning system. It was explained that selecting items in this way perhaps better fits the purpose of such a system to optimize the efficiency of individuals' learning.

The proposed method was evaluated in simulation studies with the possibility of learning growth recovery as measured by the rmse $\delta(\ell)$ expressing the accuracy by which real growth in ability between two points in time is also estimated to be there. The results clearly showed that randomly selecting items and selecting harder items, which could be motivating in learning systems, have a negative effect. The differences between the Fisher information method and the KL information method for item selection were small.

The simulation studies reported in this chapter cover only a few of the conditions that were explored in the complete study (Eggen, 2011). In these studies, the differences were also explored

- for a very large (10.000 items) one-parameter Rasch model item bank;

- for varying populations distributions with average abilities one or two standard deviations above or below the population on which was reported here and which has a mean at the mean of the difficulty of the items in the item bank;

- for varying speed in the growth during testing (small, intermediate, large);

- for three other K-L information-based selecting methods evaluated at two different ability estimates; for instance, ability estimated based on only the first items and the estimate based on all items; and

- for different maximum test lengths.

In all these conditions, the same trends were observed. In populations that grow in ability, the K-L information selection method performs better than Fisher information-based selection methods in recovering growth. The differences however are small. In 50 repeated simulations with 10.000 students, the statistical significance of the differences was not proved.

The reasons for the lack of significant improvement are not clear. Maybe there are despite the trends only significant improvements to be expected in certain conditions not studied yet. Another reason could be that all selection methods depend on the accuracy of the ability estimates. 
The K-L information-based item selection could suffer more from this than Fisher information-based selection because with K-L information two ability estimates based on only parts of the administered item sets are needed.

The first exploration of a combination of both methods consisting of using for item selection Fisher information in the beginning of the test and K-L information when at least a quarter of the total test length is administered has been conducted (Eggen, 2011). However, in this case the method performed only a tiny bit better. Nevertheless, the combination method deserves more attention.

Finally it is recommended to combine the K-L information item selection method with better estimation methods during test administration. In this context, the suggestion made by Veldkamp, Matteucci, and Eggen (2011) to improve the performance of the selection method by using collateral information about a student to get a better prediction of his or her ability level at the start of the test could be useful.

However, even more important for the practice of computerized adaptive learning system is having better continuously updated estimates of the individual's ability. The application of the dynamic ability parameter estimation approach introduced by Brinkhuis and Maris (2009) is very promising and should be considered.

\section{References}

Bergstrom, B.A., Lunz, M.E., \& Gershon, R.C. (1992). Altering the level of difficulty in computer adaptive testing. Applied Measurement in Education, 5, 137-149.

Birmbaum, A. (1968). Some latent trait models and their use in inferring an examinee's ability. In F.M. Lord \& M.R. Novick (Eds.). Statistical theories of mental test scores (pp 397-479). Reading, MA: Addison Wesley.

Brinkhuis, M.J.S. \& Maris, G. (2009). Dynamic parameter estimation in student monitoring systems. Measurement and Research Department Reports (Rep. No. 2009-1). Arnhem: Cito.

Brusilovsky, P. (1999). Adaptive and intelligent technologies for Web-based education. Künstliche Intelligenz, 13, 19-25.

Desmet, P. (2006). L'apprentisage/enseignement des langues á l'ére du numérique: tendances récentres et défis. Revue francaise de linguistique appliquée, 11, 119-138.

Eggen, T.J.H.M. (1999). Item selection in adaptive testing with the sequential probability ratio test. Applied Psychological Measurement, 23, 249-261. 
Eggen, T.J.H.M. (2011, October 4). What is the purpose of the Cat? Presidential address Second International IACAT Conference, Pacific Grove.

Eggen, T.J.H.M. \& Verschoor, A.J. (2006). Optimal testing with easy or difficult items in computerized adaptive testing. Applied Psychological Measurement, 30, 379-393.

Van der Linden, W.J. \& Glas, C.A.W. (Eds). (2010). Elements of adaptive testing. New York, Springer.

Veldkamp, B.P., Matteucci, M., \& Eggen, T.J.H.M. (2011). Computer adaptive testing in computer assisted learning. In: Stefan de Wannemacker, Geraldine Claerebout, and Patrick Decausmaeckers (Eds.). Interdisciplinary approaches to adaptive learning; a look at the neighbours. Communications in Computer and Information Science, 126, 28-39.

Wainer, H. (Ed.). (2000). Computerized adaptive testing: A primer. London: Erlbaum.

Warm, T.A. (1989). Weighted likelihood estimation of ability in item response theory. Psychometrika, 54, 427-450.

Wauters, K., Desmet, P., \& Van den Noortgate, W. (2010). Adaptive item-based learning environments based on item response theory: possibilities and challenges. Journal of Computer Assisted Learning, 26, 549-562.

Wauters, K., Desmet, P., \& Van den Noortgate, W. (2012). Disentangling the effects of item difficulty level and person ability level on learning and motivation. Submitted to Journal of Experimental Education.

Wiliam, D. (2011). What is assessment for learning? Studies in Educational Evaluation, 37, 3-14. 


\title{
Chapter 3
}

\section{Use of Different Sources of Information in Maintaining Standards: Examples from the Netherlands}

\section{Anton Béguin}

\begin{abstract}
In the different tests and examinations that are used at a national level in the Netherlands, a variety of equating and linking procedures are applied to maintain assessment standards. This chapter presents an overview of potential sources of information that can be used in the standard setting of tests and examinations. Examples from test practices in the Netherlands are provided that apply some of these sources of information. This chapter discusses how the different sources of information are applied and aggregated to set the levels. It also discusses under which circumstances performance information of the population would be sufficient to set the levels and when additional information is necessary.
\end{abstract}

Keywords: linking, random equivalent groups equating, nonequivalent groups equating

\section{Introduction}

In the different tests and examinations that are used at a national level in the Netherlands, a variety of equating and linking procedures are applied to maintain assessment standards. Three different types of approaches can be distinguished. First, equated scores are determined to compare a new form of a test to an existing form, based on an anchor that provides information on how the two tests relate in difficulty level and potentially in other statistical characteristics. A special version of this equating procedure is applied in the construction and application of item banks, in which the setting of the cut-score of a test form is based on the underlying Item Response Theory (IRT) scale. Second, in certain instances-for example, central examinations at the end of secondary education-heuristic procedures are developed to incorporate different sources of information, such as pretest and anchor test data, qualitative judgments about the difficulty level of a test, and the development over time of the proficiency level of the population. For each source of the data, the optimal cut-scores on the test are determined. Because the validity of assumptions and the accuracy of the data are crucial factors, confidence intervals around the cut-scores are determined, and a heuristic is applied to aggregate the results from the different data sources. 
Third, in the standard setting of a test at the end of primary education, significant weight is assigned to the assumption of random equivalent groups, whereas the other sources of information (pretest data, results on similar tests, and anchor information) are mainly used as a check on the validity of the equating. In the current chapter, an overview of potential sources of information that can be used in the standard setting of examinations is presented. The overview includes information on the following:

1. Linking data that can be used in equating and IRT linking procedures, with various data collection designs and different statistical procedures available

2. Different types of qualitative judgments: estimates of difficulty level/estimates of performance level

3. Assumptions made in relation to equivalent populations

4. The prior performance of the same students

5. The historical difficulty level of the test forms

Examples from test practices in the Netherlands are provided that apply some of these sources of information, which are then aggregated and applied in the standard-setting procedure to set the levels. This chapter discusses the advantages and disadvantages of some of the sources of information, especially regarding under which circumstances random equivalent groups equating - using only performance information of the population — would improve the quality or efficiency of the level-setting procedure and when additional information is necessary.

\section{Sources of Information for Standard Setting}

\section{Linking Data}

To be able to compare different forms of a test, one needs either linking data or an assumption of random equivalent groups. A number of different designs and data collection procedures have been distinguished (Angoff, 1971; Béguin, 2000; Holland \& Dorans, 2006; Kolen \& Brennan, 2004; Lord, 1980; Petersen, Kolen, \& Hoover, 1989; Wright \& Stone, 1979). In these data collection procedures, a distinction can be drawn between designs that assume that the test forms are administered to a single group or to random equivalent groups and nonequivalent group designs for which the assumption of random equivalent groups may not hold. In the context of examinations, the data collected during actual exams can theoretically be treated as data from a random equivalent groups design. 
Each form of the examination is administered to separate groups of respondents, but it is assumed that these groups are randomly equivalent. More relevant in the current context are the nonequivalent groups designs. Examples of such designs are anchor test designs, designs using embedded items, and pretest designs.

A variety of equating procedures are available to compare test forms. These procedures use the collected data to estimate the performance characteristics of a single group on a number of different test forms (e.g., to estimate which scores are equivalent between forms and how cut-scores can be translated from one form to the other).

The equating procedures either use only observable variables or assume latent variables, such as a true score or a latent proficiency variable. Procedures using only observable variables are, for example, the Tucker method (Gulliksen, 1950), Braun-Holland method (Braun \& Holland, 1982), and chained equipercentile method (Angoff, 1971). Latent variable procedures include Levine's (1955) linear true score equating procedure and various procedures based on IRT (e.g., Kolen \& Brennan, 2004; Lord, 1980).

\section{Item Banking}

Item-banking procedures can be considered a special case of equating procedures. These procedures often use a complex design to link new items to an existing item bank. They rely heavily on statistical models, the assumption that the characteristics of items can be estimated, and that these characteristics remain stable during at least a period of time. Typically, item banks are maintained by embedding new items within live test versions or by the administration of a separate pretest. If an IRT model is used, often parameters for difficulty, discrimination, and guessing are estimated. To ensure that the new items are on the same scale as the items in the bank, the new items are calibrated, together with the items for which item characteristics are available in the bank. To evaluate whether the above procedure is valid, it is crucial that the underlying assumptions are checked. For example, the stability of the items' characteristics needs to be evaluated, comparing between the previous administrations on which the item characteristics are based and the performance in the current administration. The stability can be violated in cases where items are administered under time constraints, order effects occur, or if items become known due to previous administrations.

Because of the potential adverse effect of these issues on the validity of the equating, it is crucial to monitor the performance of the individual items and the validity of the link between the new test version and the item bank. 
Clearly, the variables of interest in level setting, such as equivalent cut-scores, are directly affected by the quality and the stability of the equating procedure. The quality of the equating of test forms largely depends on potential threads to validity in the data collection. For example, the results of a pretest could potentially be biased if order effects and administration effects are not dealt with appropriately. The stability of equating depends on the quality and the size of the sample, characteristics of the data collection design, and the equating procedure that is used (e.g., Hanson \& Béguin, 2002; Kim \& Cohen, 1998).

\section{Qualitative Judgments}

To set cut-scores on a test form, standard setting procedures based on qualitative judgments about the difficulty level of the test form can be applied. Various procedures are available (e.g., Cizek, 1996, 2001; Hambleton \& Pitoniak, 2006), ranging from purely content-based procedures (Angoff procedure, bookmark procedure), which focus on the content of the test, to candidate-centered procedures (borderline, contrasting groups), which aim to estimate a cut-score based on differences between groups of candidates. For example, in a contrastinggroups procedure, raters are asked to distinguish between groups of candidates who perform below the level necessary to pass the test and groups of candidates who perform above this level. In this judgment, the raters do not use the test score. Then the test score distributions of these groups are contrasted to select the cut-score that best distinguishes between the two groups.

The quality of a level-setting procedure largely depends on the quality of the judges, the number of judges involved, the characteristics of the procedure, and the quality of the instruction. Often, relatively unstable or biased results are obtained in cases where the instruction or the number of judges is insufficient.

\section{Random Equivalent Groups}

In contrast to many other sources of equating information, the performance level of the population is often a very stable measure. Comparing the performance level of the population between one year and the next will only result in large differences if the composition of the population or the curriculum has changed. Differences in year-to-year performance could also occur if there is an increasing or decreasing trend in performance. However, in a number of cases, it is not unreasonable to make an assumption of random equivalent groups from one year to the next. Based on this assumption, it is possible to apply level-setting procedures. 
An extended version of the assumption of random equivalent groups takes background variables into account. If the year-to-year populations differ in composition based on a number of background variables, this difference can be corrected using weighing. In such cases, groups of students with the same background variable are assumed to be a random sample from the same population. Using weighing based on background variables, the assumption of random equivalent groups will hold again in the total population.

\section{Prior Performance of the Same Group}

Procedures used to estimate the performance level based on prior attainment on a test a few years earlier can be viewed as a special case of taking background information into account. Two pieces of information can be derived from the prior attainment data: On one hand, the data show whether the population deviates from the average. A correction for this would be similar to the extended assumption of random equivalent groups described above. On the other hand, the prior attainment data could provide information on the performance levels that were reached earlier. Using the information on how the prior performance relates to the standards on the new test form, the cut-scores on this new form can be estimated.

\section{Historical Difficulty Level of the Test Forms}

The variation in the difficulty level of the test forms constructed according to the same test blueprint can be used to estimate the difficulty of the current test form. Assuming that the current test form will not be significantly different from the previous forms (e.g., over the past 10 years) will result in a confidence interval. Using historical information, it is assumed that the difficulty of this year's form will fall within this confidence interval.

\section{Linking Procedures Used in Some of the Principal Tests in the Netherlands}

\section{Entrance Test to Teacher Training}

During the first year of the teacher training program, students have to pass tests in mathematics and in the Dutch language. Students will have a maximum of three opportunities to pass these tests. If they fail these attempts, they are not allowed to continue their education.

The mathematics test is an adaptive test based on an underlying item bank calibrated using the one-parameter logistic model (OPLM) (Verhelst, Glas, \& Verstralen, 1994). The item parameters are based on samples with at least 600 respondents for each item in the bank. In addition to the data on the respondents from the teacher training program, these samples may also include information collected from other fields of education. 
The bank may contain, for example, items that originated in primary education. In such cases, the original item parameters are based on the performance of students in primary education. On a yearly basis, the parameters are updated based on the performance during the actual administration of the test. New items are pretested on a yearly basis to enable collection of the necessary data to estimate the item parameters on the same scale as the other in the bank.

\section{Examinations at the End of Secondary Education}

At the end of secondary education, the students take a set of final examinations in a number of subjects that they selected earlier. After passing these examinations, they gain access to different forms of further education. The final examinations in most subjects are divided into two parts: a school examination and a national examination. The elements that are tested in each examination are specified in the examination syllabus, which is approved by the College voor Examens (CVE) (English translation: Board of Examinations, an arm's length body of the Ministry of Education). The CVE is also responsible for the level setting of the examinations. In the majority of examinations, the level-setting procedure is dominated by the information obtained using the assumption of random equivalent groups. Some other examinations have a small number of candidates; consequently, there is insufficient information about the performance of candidates. In such cases, a content judgment is used as the basis for the level setting. More elaborate data collection provides extra information for specific examinations considered central to the examination system. These include examinations in basic skills (Dutch language and mathematics), modern languages (English, French, and German), science (physics, chemistry, and biology), and economics. For these examinations, the additional data are collected using a pretest or posttest design (Alberts, 2001; Béguin, 2000). In these designs, parts of past and future examination forms are combined into tests that are administered as a preparation test for the examination. In other instances, the data are collected in different streams of education. Based on the collected data and using a Rasch model, the new examination is linked to an old form of the test. In this way, the standard on the new form can be equated to the standard on the old form.

The amount of data collected in the pretest or the posttest design is relatively limited due to restrictions on security of the items. Consequently, the equated score is provided with a confidence interval. As input to the level-setting meeting, the results of the above linking procedure are combined with the results of linking based on an assumption of random equivalent groups from year to year and, in some cases, content-based judgements about the difficulty level of the examinations. 


\section{End of Primary School Test}

At the end of primary education, schools are obliged to collect objective information about the most appropriate type of secondary education for students. Most of the schools (about 85\%) apply a test for this purpose called the Eindtoets Basisonderwijs (Cito, 2012; Van der Lubbe, 2007), whereas the remainder apply other tests and assessments.

The Eindtoets Basisonderwijs contains a compulsory section composed of 200 multiplechoice items on the Dutch language, as well as on arithmetic and study skills, and a voluntary section composed of 90 items on history, geography, and science. Each year, a new form of the test is constructed that contains only new items, and the results are linked to those of the previous year's test. Three linking procedures based on different sources of information are used in the standard setting in the Eindtoets Basisonderwijs. The linking procedures are based on the following:

1) Pretest data in which the pretest forms combine items from multiple test forms of different years in a complex incomplete design

2) Anchor data that are collected, using an internal anchor embedded within the test forms of a sample of approximately 3,000 pupils taking the test, and noting that the anchor counts to the final score of these pupils

3) An assumption of random equivalent groups based on a sample of 1,800 schools that participated in the test for the past four years and in which no large shifts in the performance or in the size of the school occurred in this period

In the pretest equating, a multidimensional equating procedure is applied in which each of the 13 domains in the test is modelled using a separate dimension that is correlated to the other dimensions (Béguin, 2000; Glas, 1989).

Equating based on the pretest data is relatively unstable due to the small sample size of approximately 600 pupils per item and its susceptibility to model imperfections when, for example, order effects or time constraints are present.

The conditions under which the test is administered pose an additional threat to the validity of the linking, i.e., often the stakes are low for the student because the outcome of the test will have less importance to the student than the actual test. The administration condition could have an effect on the motivation of the pupils and, therefore, result in bias of the linking. 
In the pretest, we tried to diminish this effect by collecting data in such a way that the motivation of the students was similar for all the items. However, this step does not guarantee that motivational effects will have no effect on the pretest equating. Equating using an anchor test is far more robust due to its larger sample size and the fact that the test is administered under high-stakes conditions. In addition, the design is simpler. Thus, potential problems associated with time pressure and order effects are more easily detected and addressed.

A potential drawback with the anchor test design is that the anchor becomes known, and this will result in an increase in performance on the anchor that does not reflect an actual increase in proficiency. Finally, equating based on an assumption of random equivalent groups is stable and robust if the composition of the population does not change over time. A potential drawback of this approach is that if changes in the performance of the population do occur, they will be ignored.

Over the past few years, significant weight has been given to the assumption of random equivalent groups (Van Boxtel, Engelen, \& De Wijs, 2012). This is because the standard setting based on the other sources of information (pretest data and anchor information) is, to some extent, inaccurate, whereas the trends in performance over time are historically stable. For reporting at the student level, it is unlikely that the standard setting based on the assumption of random equivalent groups compromises the standard because year-to-year effects are very small compared with the differences among students. However, to ensure that potential trends in performance are detected, all other sources of linking data are analyzed and used as a check on the standard setting. The results of these analyses are published in a report on the performance at the system level, which is available to the public a few months later. This report includes the results based on the different sources of linking information, together with the confidence intervals and corrected for background variables.

The type of detail that can be provided in such a report cannot be incorporated in the operational standard setting because of time constraints and the impossibility of including uncertainty in the reported cut-score.

\section{Maintaining Performance Using Random Equivalent Groups Equating Instead of Maintaining Standards Using Nonequivalent Group Designs?}

A number of equating procedures have been described earlier in the chapter, with some examples provided from tests in the Netherlands. In the level setting of both the central examinations in secondary education and in the end of primary school test, it is considered crucial that the standards are maintained over time. 
In contrast to this, operationally the level setting depends at least partly on random equivalent groups equating, which theoretically just maintains performance. The reason for this seemingly invalid procedure is that in these tests the expected difference in performance level between the years is expected to be smaller than the standard error of the equating procedures used to maintain the standard.

As a consequence, maintaining performance will be expected to reduce the instability of the level setting in a single year by trading the potential instability of the equating procedure for the potential bias due to the assumption of random equivalent groups.

According to the argument above, random equivalent groups equating could theoretically be used as the only source of information for level setting for these tests. However, there is a drawback for the maintaining-performance-only approach: Over multiple years, the bias would accumulate if a trend in performance in the population would occur. Another drawback with this approach is that maintaining performance could potentially undermine trust in the assessment system if the public considers that this procedure leads to a decrease in performance.

To be able to respond to claims about decreasing performance, it is crucial that a trend in performance can be evaluated at the system level and that standards can be maintained at that level. Therefore, next to maintaining performance based on random equivalent groups equating, equating information using additional data (like pretest and anchor test) also needs to be available, such as is the case in the examinations in secondary education and the test at the end of primary education. According to the additional data, it is possible to report on trends in performance in a detailed and nuanced way.

For example, it is possible to publish results, together with a confidence interval, or to report on different sources of equating information that contradict each other. Operationally, reporting in this level of detail is possible only at the system level, because uncertainty about standards cannot be included in a practical way in reports at pupil and school levels. In practice, some situations will present a difference between the cut-scores based on random equivalent groups equating and used for pupils and schools and the reported results of the performance in relation to the standards that include more sources of information.

In these cases, a correction will need to be made to the basis used for comparison in the test administered in the following year. This will prevent the accumulation of differences over the years from compromising the standard. 
In summary, using a system based on maintaining performance (using random equivalent groups equating), combined with a number of equating procedures that are not necessarily all used as direct input in level setting, seems operationally to be the best option in circumstances where the expected differences in performance from year to year are smaller than the expected standard error of the equating procedures. The result from the equating procedures will be used in analyses at the system level to report on trends in performance in a detailed and nuanced way.

Although this procedure will potentially lead to a (probably small) deviation from the standard at the individual and school levels each year, the use of this approach over a number of years will not necessarily result in the accumulation of bias.

\section{References}

Alberts, R. V. J. (2001). Equating exams as a prerequisite for maintaining standards: Experience with Dutch centralised secondary examinations. Assessment in Education: Principles, Policy \& Practice, 8, 353-367.

Angoff, W. H. (1971). Scales, norms and equivalent scores. In R. L. Thorndike (Ed.), Educational measurement (2nd ed., pp. 508-600). Washington, DC: American Council on Education.

Béguin, A. A. (2000). Robustness of equating high-stakes tests. $\mathrm{PhD}$ thesis, University of Twente, Enschede.

Braun, H. I., \& Holland, P. W. (1982). Observed-score test equating: A mathematical analysis of some ETS equating procedures. In P. W. Holland \& D. B. Rubin (Eds.), Test equating (pp. 9-49). New York: Academic Press.

Cito (2012).Handleiding Eindtoets Basisonderwijs [Manual End of Primary School Test], Arnhem, the Netherlands: Cito.

Cizek, G. J. (1996). Setting passing scores. Educational Measurement: Issues and Practice, $15,20-31$.

Cizek, G. J. (2001). Setting performance standards: Concepts, methods, and perspectives. Mahwah, NJ: Erlbaum.

Glas, C. A. W. (1989). Contributions to estimating and testing Rasch models. (Doctoral Thesis.) Enschede: University of Twente.

Gulliksen, H. (1950). Theory of mental tests. New York: Wiley. 
Hambleton, R. K., \& Pitoniak, M. (2006). Setting performance standards. In R. L. Brennan (Ed.), Educational measurement (pp. 433-470). Westport, CT: American Council on Education.

Hanson, B. A., \& Béguin, A. A. (2002). Obtaining a common scale for IRT item parameters using separate versus concurrent estimation in the common item nonequivalent groups equating design. Applied Psychological Measurement, 26, 3-24.

Holland, P. W., \& Dorans, N. J. (2006). Linking and equating. In R. L. Brennan (Ed.), Educational measurement (4th ed.). Westport, CT: American Council on Education and Praeger Publishers.

Kim, S., \& Cohen, A. S. (1998). A comparison of linking and concurrent calibration under item response theory. Applied Psychological Measurement, 22, 131-143.

Kolen, M. J., \& Brennan, R. L. (2004). Test equating (2nd ed.). New York: Springer.

Levine, R. E. (1955). Equating the score scales of alternative forms administered to samples of different ability. Research Bulletin 55-23, Educational Testing Services, Princeton,NJ

Lord, F. M. (1980). Applications of item response theory to practical testing problems. Hillsdale, NJ: Lawrence Erlbaum Associates.

Petersen, N. S., Kolen, M. J., \& Hoover, H. D. (1989). Scaling, norming and equating. In R. L. Linn (Ed.), Educational measurement (3rd ed. ,pp. 221-262). New York: American Council on Education and Macmillan.

Van Boxtel, H., Engelen, R., \& De Wijs, A. (2012). Verantwoording van de Eindtoets Basisonderwijs 2010. Arnhem: Cito.

Van der Lubbe, M. (2007). The End of Primary School Test (better known as Citotest). Paper presented at the 33rd annual conference of the International Association for Educational Assessment, September 16-21, Baku, Azerbaijan.

Verhelst, N. D., Glas, C. A. W., \& Verstralen, H. H. F. M. (1994). OPLM: Computer program and manual. Arnhem: Cito.

Wright, B. D., \& Stone, M. H. (1979). Best test design. Chicago: MESA Press University of Chicago. 


\title{
Chapter 4
}

\section{Ensuring the Future of Computerized Adaptive Testing}

\section{Bernard P. Veldkamp}

\begin{abstract}
Capitalization on chance is a huge problem in computerized adaptive testing (CAT) when Fisher information is used to select the items. Maximizing Fisher information tends to favor items with positive estimation errors in the discrimination parameter and negative estimation errors in the guessing parameter. As a result, information in the resulting tests is overestimated and measurement precision is lower than expected. Since reduction of test length is one of the most important selling points of CAT, this is a serious threat to both the validity and viability of this test administration mode. In this chapter, robust test assembly is presented as an alternative method that accounts for uncertainty in the item parameters during test assembly.
\end{abstract}

Keywords: Automated test assembly, capitalization on chance, computerized adaptive testing, item parameter uncertainty, 0-1 LP, robust test assembly

\section{Introduction}

In computerized adaptive testing (CAT), item administration is tailored to the test taker. Tailoring the test turns out to entail a number of advantages. The candidate only has to answer items that are paired to his or her ability level, test length can be reduced, and test administration can be more flexible as a result of individualized testing. Besides, CATs could be offered continuously, on flexible locations, and even via the Web. The advantages of CAT turned out to be very appealing. Nowadays many CATs are run operationally in educational, psychological, and health measurement. Various algorithms for tailoring the test have been proposed. They generally consist of the following steps:

1. Before testing begins, the ability estimate of the candidate is initialized (e.g., at the mode of the ability distribution, or based on historical data). 
2. Items are selected from an item bank to be maximally informative at the current ability estimate. Sometimes, a number of specifications related to test content or other attributes have to be met, which restricts the number of items available for selection. In this step, an exposure-control method is commonly applied to prevent overexposure of the most popular items.

3. Once an item is selected, it is administered to the candidate.

4. An update of the ability estimate is made after each administration of an item.

5. Finally, the test ends whenever a stopping criterion has been met, for example when a fixed number of items have been administered or when a minimum level of measurement precision has been obtained.

One of the assumptions underlying these CAT algorithms is that, for all items in the bank, the item parameters are known and can be treated as fixed values during test administration to calculate the amount of information provided. Unfortunately, this assumption is never met in practice. Item parameters have been estimated based on finite samples of candidates. The estimates might be unbiased, but they still have measurement error in them. This uncertainty is a source of concern. When test information is maximized, those items with high discrimination parameters will be selected from the bank. Positive estimation errors in the discrimination parameters will increase the amount of information provided, and therefore will increase the probability that the item will be selected. This phenomenon is also referred to as the problem of capitalization on chance.

Hambleton \& Jones (1994) were among the first to study the effects of item parameter uncertainty on computerized construction of linear test forms from calibrated item banks. They found out that not taking the uncertainty into account resulted in serious overestimation of the amount of information in the test. Veldkamp (2012) illustrated this effect when he simulated an item bank of 100 items with uncertainty in them. All 100 items had the same parent, that is, all item parameters were drawn from the same multivariate distribution $N(\mu, \Sigma)$ , with $\mu$ equal to the true item parameters $(a=1.4, b=0.0, c=0.2)$ and $\Sigma$ being the diagonal matrix with the standard errors of estimation ( $S E a=0.05, S E b=0.10, S E c=0.02$ ). As a result the item parameters only varied due to uncertainty in the parameter estimates. Parameter ranges were $a \in[1.29,1.52], b \in[-0.31,0.29]$, and $c \in[0.14,0.28]$. Ten items with highest Fisher information at $\theta=0.0$ were selected from this bank for a test. 
The resulting test information function was compared to the test information function based on the true item parameters $(a=1.4, b=0.0, c=0.2)$. As can be seen in Figure 1, the test information is overestimated by $20 \%$, when uncertainty is not taken into account.

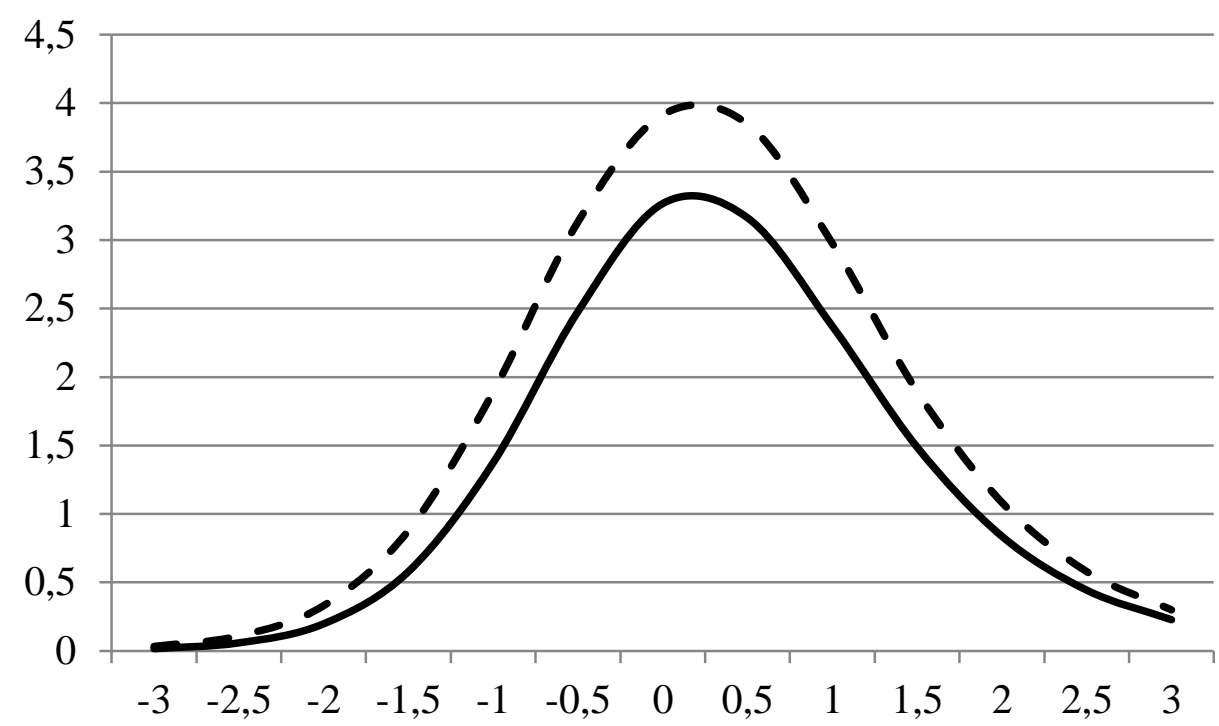

Figure 1 Test information function: ATA (dashed line) or true (solid line)

Hambleton \& Jones (1994) demonstrated that the impact of item parameter uncertainty on automated construction of linear tests depended on both the calibration sample size and the ratio of item bank size to test length. When their findings are applied to CAT, calibration sample size plays a comparable role. The ratio of item bank size to test length is more of an issue in CAT, since only one item is selected at a time, which results in an even less favorable ratio. Olea, Barrada, Abad, Ponsoda, \& Cuevas (2012), studied the impact of capitalization on chance for various settings of CAT in an extensive simulation study, and they confirmed the observations of Hambleton and Jones (1994). In other words, capitalization on chance is a huge problem in CAT when Fisher information is used to select the items. The measurement precision of the test is vastly overestimated. Alternative strategies for item selection in CAT will have to be used so as not to compromise the validity of this test administration mode.

\section{Robust Test Assembly}

In combinatorial optimization, mathematical techniques are applied to find optimal solutions within a finite set of possible solutions. 
The set of possible solutions is generally defined by a set of restrictions. Automated test assembly (ATA) problems are a special case of combinatorial optimization problems. The objective of ATA is often to maximize the information in the test, and the set of possible solutions is generally defined by the test specifications, for example, by the content constraints. An extensive introduction to the topic of formulating ATA problems as mixed integer programming (MIP) problems can be found in van der Linden (2005).

To solve the problem of dealing with uncertainty in the item parameters in CAT, a first step would be to search the literature for methods that have been proposed to deal with parameter uncertainty in combinatorial optimization. Soyster (1973) was among the first to present a method for dealing with uncertainty in combinatorial optimization problems. $\mathrm{He}$ assumed that for every uncertain parameter an interval could be defined that contained all possible values. He replaced each uncertain parameter by its infimum and solved the problem. This solution served as a robust lower bound for the solution of the original problem. Unfortunately, this method was very conservative. It assumed a maximum error in all the parameters, which is highly unlikely in practice. The good thing, however, was that Soyster (1973) opened up a new area of research: robust optimization. The ultimate goal of robust optimization (Ben Tal, El Ghaoui, \& Nemirovski, 2009) is to take data uncertainty into account when the optimization problem is solved in order to "immunize" resulting tests against this uncertainty. Under this approach, a suboptimal solution is accepted in order to ensure that the solution remains near optimal when the estimated parameters turn out to differ from their real values. For ATA this means that uncertainty in the item parameters or in the information function is taken into account during test assembly to immunize the test against overestimation of the test information.

De Jong, Steenkamp, \& Veldkamp (2009) applied a modified version of Soyster's method to ATA,when they constructed country-specific versions of a small marketing scale. Instead of replacing uncertain parameters by their infima, they subtracted one posterior standard deviation from the estimated Fisher information as a robust alternative. Veldkamp, Matteucci, \& de Jong (2012) studied this modified Soyster method in more detail, for example, they studied differences in effects of uncertainties in various item parameters in test assembly. 
Veldkamp (2012) studied a different approach based on the robust optimization method developed by Bertsimas and Sim (2003). Instead of doing a small correction (minus one standard deviation of the uncertainty distribution) for all items in the bank, a substantive correction (replacing the parameters by their infima) is made only for the maximum number of items assumed to affect the solution.

This resembles more closely the practice of ATA, where some items in the test will have high positive estimation errors, while others will not. A robustness level $\Gamma$ (i.e., the maximum number of item parameters that might be replaced) has to be defined beforehand. $\Gamma$ can vary anywhere from zero (which resembles ATA) to all items in the test (which resembles the Soyster method). When the ratio of item bank size to test length is small, many items will be selected from the item bank. $\Gamma$ will be close to zero, because only a few of the selected items will have high positive estimation errors. When the ratio of item bank size to test length is high, only a very small proportion of the items will be selected from the bank. Capitalization of chance will be more of an issue, and $\Gamma$ will be closer to the test length. Bertsimas and Sim (2003) proved that finding an optimal solution for a combinatorial optimization problem where at most $\Gamma$ parameters were allowed to change, was equal to solving $(\Gamma+1)$ MIP problems. For details of the method, see Veldkamp (2012).

\section{Robust CAT Based on Expected Information}

Even though relatively good results were obtained for some practical test assembly problems with the modified Soyster method (see de Jong et al., 2009) and the Bertsimas and Sim method (see Veldkamp, 2012), both methods do not use information known about the distribution of the item parameter uncertainty. Uncertainty in the item parameters results from parameter estimation, and it is assumed to follow a normal distribution with a mean equal to the parameter estimates and a standard deviation equal to the standard error of estimation for maximum likelihood estimation, or to the posterior standard deviation in a Bayesian framework. This information could be used to calculate the expected information for each item, taking the uncertainty distribution of the parameters into account. Lewis (1985) already proposed using expected response functions (ERFs) to correct for uncertainty in the item parameters (Mislevy, Wingersky, \& Sheehan, 1994) for fixed-length linear tests. The same idea might be applied at the item bank level as well, thus providing a starting point for a robust test assembly procedure for CAT. 


\section{Robust Item Pool}

The first step in such a procedure would be to develop a robust item pool. Since the uncertainties in the parameters are assumed to follow a normal distribution, the cumulative distribution function can be used to calculate which percentage of the items is expected to have which deviation. For example, $2.5 \%$ of the items are assumed to have a positive deviation larger than 1.96 standard deviations.

Based on this information, robust item information can be calculated by subtracting the expected deviation from the estimated item information. When all items in the bank are ordered from smallest to largest with respect to their maximum information, the robust item information can be calculated as:

$$
I_{i}^{R}(\theta)=I_{i}(\theta)-z_{i} * S D\left(I_{i}(\theta)\right), \quad i=1, \ldots, I,
$$

where $i$ is the index of the item in the ordered bank, $I$ is the number of items in the bank, $I_{i}^{R}(\theta)$ is the robust information provided at ability level $\theta, z_{i}$ corresponds to the $100 \cdot i /(I+1)$ -th percentile of the cumulative normal distribution function, and $\operatorname{SD}\left(I_{i}(\theta)\right)$ is the standard deviation of the information function based on estimated item parameters. Within a Bayesian framework, a comparable procedure has to be applied, where the posterior distribution is used to calculate $z_{i}$.

\section{Empirical Example}

To illustrate the effects of expected information, robust item information was calculated for all items of an operational item bank. 306 items were calibrated with a three-parameter logistic model (3PLM):

$$
P_{i}(\theta)=c+(1-c) \frac{e^{a(\theta-b)}}{1+e^{a(\theta-b)}}
$$

where $a$ is the discrimination, $b$ is the difficulty, and $c$ is the guessing parameter. The item parameters were estimated using BILOG MG 3, for a sample of 41,500 candidates. The estimated parameter ranges were $a \in[0.26,1.40], b \in[-3.15,2.51]$, and $c \in[0.00,0.50]$, and the average uncertainties were $(\Delta a=0.02, \Delta b=0.044, \Delta c=0.016)$. 
The maximum amount of information over all theta levels (Hambleton, \& Swaminathan, 1985, p.107) provided by the 50 most informative items is shown in Figure 2.

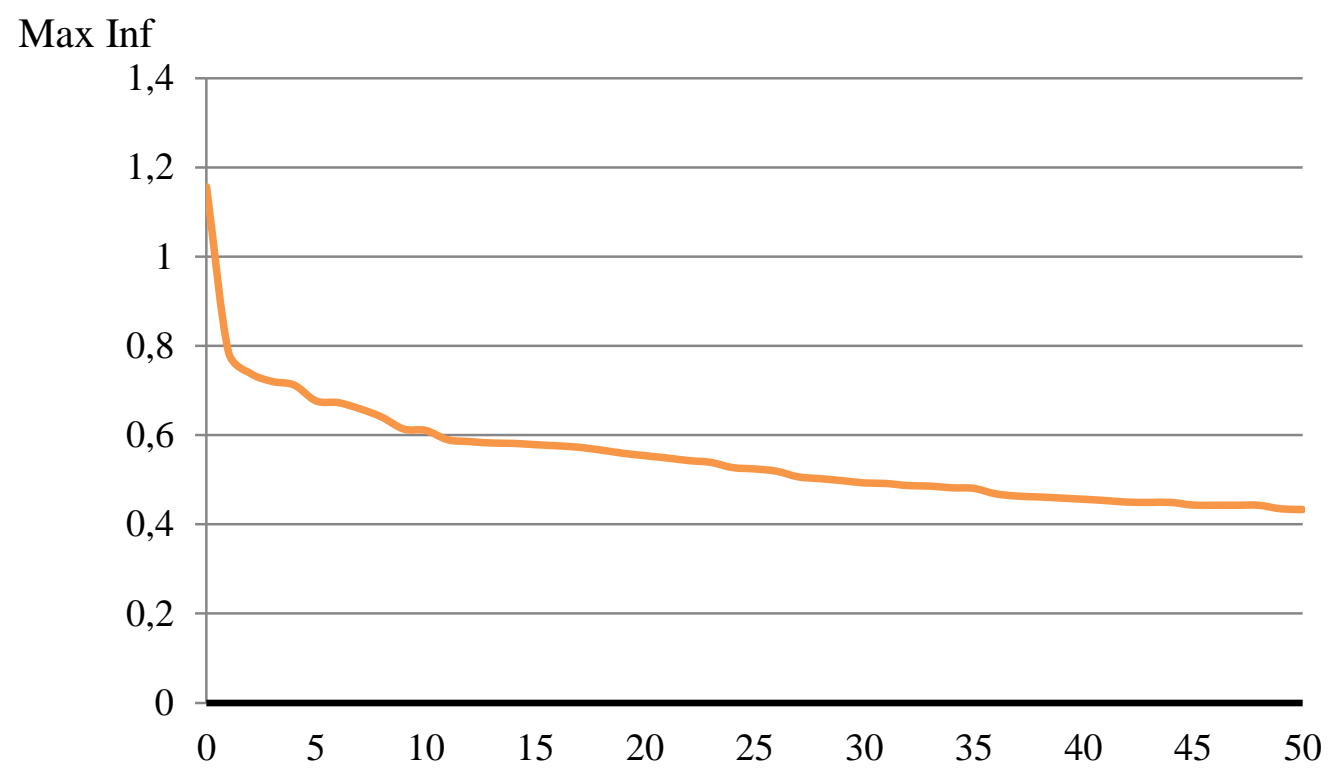

Item no.

Figure 2 Maximum amount of information provided by the 50 most informative items

All items were ranked with respect to their maximum amount of information over all theta levels, and the robust information was calculated by subtracting the expected deviation for all of the items. To illustrate how robust item information corrects for uncertainty in the item parameters, its performance was compared with a number of simulated item banks. Three item banks were simulated by randomly drawing item parameters from the multivariate normal distribution with a mean equal to the estimated item parameters and standard deviations equal to the errors of estimation. The deviance in maximum information between the estimated item parameters on the one hand and the robust and simulated item parameters on the other hand is shown in Figure 3. 
Deviation

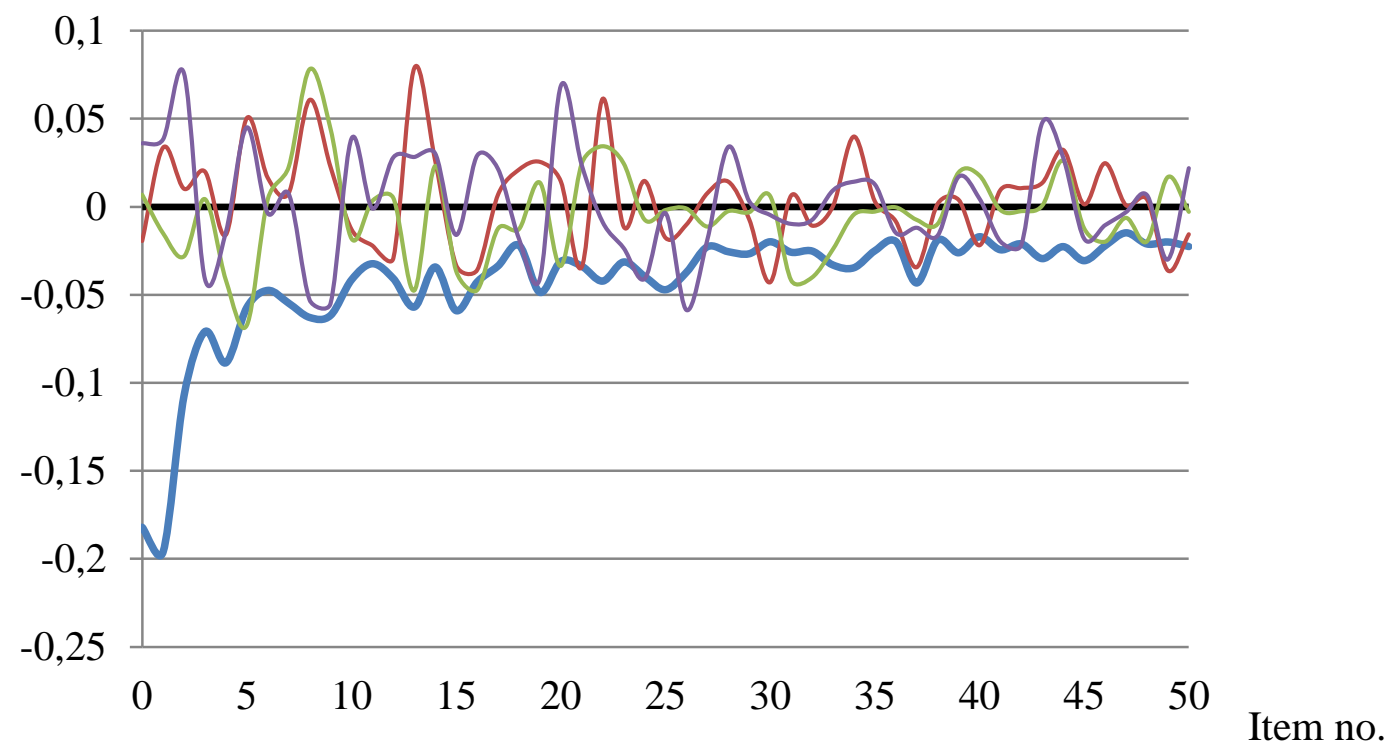

Figure 3 Deviations from the maximum information for the robust information (thick line) and various simulated item banks (thin lines) for the 50 most informative items

As expected, the robust maximum information is generally smaller than the estimated maximum information for the 50 most informative items, but the difference becomes smaller and smaller when the items are less informative. Because of the differences in $S D(I(\theta))$ for the various items, the robust maximum information does not increase monotonically. As can be seen in Figure 3, $S D(I(\theta))$ for the second item is larger than $S D(I(\theta))$ for the first item. The curves of the deviances for the simulated item banks hover around zero. By chance, the deviation will be positive for some of the items and negative for others. It can also be seen that for individual items, the deviance for the simulated information could even be larger than the deviation of the robust information, but for a test, which is for a group of items, the robust maximum information serves pretty well as a lower bound.

\section{Robust Item Selection}

The robust item information is still conservative. It assumes that uncertainty hits where it hurts most; that is, it assumes that the most informative items have the highest uncertainty in them. In practice, however, this is not the case. This can also be seen in Figure 3, where for the first 25 items, the robust maximum information is obviously smaller than the simulated maximum information. To correct for this conservatism, the Bertsimas and Sim method can be applied for item selection in the second step of robust CAT. 
This method assumes that uncertainty only affects the solution for at most $\Gamma$ items in the test. The following pseudo-algorithm describes the application of the Bertsimas and Sim method for selecting the $g^{\text {th }}$ item in CAT for a fixed length test of G items:

1. Calculate $d_{i}=I_{i}\left(\theta^{g-1}\right)-I_{i}^{R}\left(\theta^{g-1}\right)$ for all items.

2. Rank the items such that $d_{1} \geq d_{2} \geq \ldots \geq d_{n}$

3. For $l=1, \ldots,(G-(g-1))+1$ find the item that solves:

$$
G^{l}=\max \left\{\sum_{i=1}^{I} I_{i}\left(\hat{\theta}^{g-1}\right) x_{i}-\left[\sum_{i=1}^{l}\left(d_{i}-d_{l}\right) x_{i}+\min (G-g, \Gamma) d_{l}\right]\right\}
$$

subject to:

$$
\begin{gathered}
\sum_{i \in R^{g-1}} x_{i}=g-1 \\
\sum_{i=1}^{I} x_{i}=g \\
x_{i} \in\{0,1\} \quad i=1, \ldots, I .
\end{gathered}
$$

4. Let $l^{*}=\arg \max _{l=1, \ldots, n} G^{l}$.

5. Item $g$ is the unadministered item in the solution of $G^{l^{*}}$.

In step 3 of the pseudo algorithm, $(\mathrm{G}-(\mathrm{g}-1))+1 \mathrm{MIP}$ are solved, where $(\mathrm{G}-(\mathrm{g}-1))$ is the amount of items still to be selected. For the MIPs, it holds that $x_{i}$ denotes whether item $i$ is selected $\left(x_{i}=1\right)$ or not $\left(x_{i}=0\right)$ (see also Equation [6]), and $R^{g-1}$ is the set of items that have been administered in the previous $(g-1)$ iterations. 
Equations (4)-(5) ensure that only one new item is selected. Finally, in (3) the amount of robust information in the test is maximized. This objective function consists of a part where the information is maximized and a part between square brackets that corrects for overestimation of the information.

This correction term varies for each value of $l=1, \ldots,(G-(g-1))+1 . \quad d_{l}$ represents the overestimation of the information in item $l$. When $l=1, d_{l}$ is equal to the largest overestimation of item information at the estimated ability level in the item bank, and $\Gamma$ times $d_{1}$ (or $(\mathrm{G}-(\mathrm{g}-1)) d_{1}$, when less than $\Gamma$ items are remaining) is subtracted as a correction. This will be too conservative because there is only one item with the maximum overestimation of the information. For larger values of $l$, the amount of overestimation is smaller, which implies that the correction factor is smaller, and the solution is less conservative.

For these values of $l$ it is taken into account that selecting one of the items with $i<l$ results in a larger overestimation, since, as a result of the ordering in step $2, d_{i}>d_{l}$. By solving (G-(g-1))+1 MIPs and choosing the maximum, a robust alternative for the test information that is not too conservative can be calculated. For details and proofs see Veldkamp (2012) and Bersimas \& Sim (2003).

\section{Conclusion and Discussion}

Capitalization on chance is a serious problem in CAT that might negatively affect both the validity and viability of this test administration mode. In this chapter, the outline of a procedure for robust CAT was presented as an answer to this problem. It accepts a suboptimal solution that remains near optimal even when item parameters turn out to be seriously overestimated. The next step in this research would be to carry out an extensive simulation study to determine its strengths and weaknesses.

Other methods have been proposed in the literature to deal with the problem of capitalization on chance. Belov and Armstrong (2005) proposed using an MCMC method for test assembly that imposes upper and lower bounds on the amount of information in the test. Since there is no maximization step in their approach, item selection is not affected by the capitalization on chance problem. On the other hand, this approach does not take uncertainty in the item parameters into account at all. This could lead to infeasibility problems (Huitzing, Veldkamp, \& Verschoor, 2005), as illustrated in Veldkamp (2012). Besides, MCMC test assembly was developed for the assembly of linear test forms, and therefore application to CAT is not straightforward. 
Olea et al. (2012) propose using item exposure control to deal with this problem. When items are selected based on maximum information, the most informative items tend to be selected more often than the others. Exposure-control methods can be implemented to limit item exposure and force less informative items to be selected. In this way, selection of the most informative items due to capitalization on chance will be prevented. Olea et al. (2012) report some promising results. Instead of correcting for uncertainty, this method limits the probability that items most vulnerable to overestimation of their information will be selected. A combination of robust CAT and item exposure control would probably result in a very strong method to prevent the capitalization on chance problem in CAT.

Every operational CAT program seriously needs to consider the impact of uncertainty in the item parameters on the reported measurement precision. Various simulation studies by Hambleton and Jones (1994), Olea et al. (2012), Veldkamp (2012), and Veldkamp et al. (2012) reported overestimation of the amount of information in the test of up to $40 \%$. When the uncertainty in the item parameters is known, simulation studies have to be carried out to determine the impact on the specific CAT program at hand. Once the impact is known, one can decide either to neglect the problem or to implement a method that deals with item parameter uncertainty either implicitly (by applying exposure-control methods) or explicitly by using robust CAT, or by a combination of both.

\section{Acknowledgement}

This chapter was written when the author was a visiting scholar at the Law School Admission Council (LSAC). The opinions and conclusions contained in the chapter are those of the author and do not necessarily reflect the positions or policy of LSAC.

\section{References}

Belov, D. I., \& Armstrong, D. H. (2005). Monte Carlo test assembly for item pool analysis and extension. Applied Psychological Measurement, 29, 239-261. DOI:10.1177/0146621605275413

Ben-Tal, A., El Ghaoui, L., \& Nemirovski, A. (2009). Robust optimization. Princeton, NJ: Princeton University Press.

Bertsimas, D., \& Sim, M. (2003). Robust discrete optimization and network flows. Mathematical Programming, 98, 49-71, DOI:10.1007/s10107-003-0396-4 
De Jong, M. G., Steenkamp, J.-B. G. M., \& Veldkamp, B. P. (2009). A model for the construction of country-specific yet internationally comparable short-form marketing scales. Marketing Science, 28, 674-689. DOI:10.1287/mksc.1080.0439

Hambleton, R. H., \& Jones, R. W. (1994). Item parameter estimation errors and their influence on test information functions. Applied Measurement in Education, 7, 171186. DOI 10.1207/s15324818ame0703_1

Hambleton, R.H., \& Swaminathan, H. (1985). Item Response Theory, Principles and Applications. Boston, MA: Kluwer Nijhoff Publishing

Huitzing, H. A., Veldkamp, B. P., \& Verschoor, A. J. (2005). Infeasibility in automated test assembly models: A comparison study of different methods. Journal of Educational Measurement, 42, 223-243. DOI:10.1111/j.1745-3984.2005.00012.x

Lewis, C. (1985). Estimating individual abilities with imperfectly known item response functions. Paper presented at the Annual Meeting of the Psychometric Society, Nashville, TN.

Mislevy, R. J., Wingersky, M. S., \& Sheehan, K.M. (1994). Dealing with uncertainty about item parameters: Expected response functions (Research Report 94-28-ONR). Princeton, NJ: Educational Testing Service.

Olea, J., Barrada, J. R., Abad, F. J., Ponsoda, V., \& Cuevas, L. (2012). Computerized adaptive testing: The capitalization on chance problem. The Spanish Journal of Psychology, 15, 424-441. DOI:10.5209/rev_SJOP.2012.v15.n1.37348

Soyster, A.L. (1973). Convex programming with set-inclusive constraints and applications to inexact linear programming. Operations Research, 21. 1154-1157.

Van der Linden, W. J. (2005). Linear models for optimal test design. New York: Springer Verlag.

Veldkamp, B. P. (2012). Application of robust optimization to automated test assembly (Research Report 12-02). Newtown, PA: Law School Admission Council.

Veldkamp, B. P., Matteucci, M., \& de Jong, M. (2012). Uncertainties in the item parameter estimates and robust automated test assembly. Manuscript submitted for publication. 


\title{
Chapter 5
}

\section{Classifying Unstructured Textual Data Using the Product Score Model: An Alternative Text Mining Algorithm}

\author{
Qiwei He and Bernard P. Veldkamp
}

\begin{abstract}
Unstructured textual data such as students' essays and life narratives can provide helpful information in educational and psychological measurement, but often contain irregularities and ambiguities, which creates difficulties in analysis. Text mining techniques that seek to extract useful information from textual data sources through identifying interesting patterns are promising. This chapter describes the general procedures of text classification using text mining and presents an alternative machine learning algorithm for text classification, named the product score model (PSM). Using the bag-of-words representation ( single words), we conducted a comparative study between PSM and two commonly used classification models, decision tree and naïve Bayes. An application of these three models is illustrated for real textual data. The results showed the PSM performed the most efficiently and stably in classifying text. Implications of these results for the PSM are further discussed and recommendations about its use are given.
\end{abstract}

Keywords: text classification, text mining, product score model, unstructured data

\section{Introduction}

Language is magic that diversifies our lives. The way individuals talk and write provides a window into their emotional and cognitive worlds. Yet despite the interesting attributes of textual data, analyzing them is not easy. One of the major reasons is that textual data are generally more diverse than numerical data and are often unstructured, neither having a predefined data model nor fitting well into relational patterns. The irregularities and ambiguities make it even harder to classify textual data compared with structured data stored in field form in databases. Thus, to address the challenge of exploiting textual information, new methods need to be developed.

The development of information technology demonstrated breakthroughs in handling unstructured textual data during the past decade. A promising technique is text mining, which exploits information retrieval, information extraction, and corpus-based computational linguistics. Analogous to data mining, text mining seeks to extract useful information from textual data sources by identifying interesting patterns. 
However, a preprocessing step is required to add transforming unstructured data stored in texts into a more explicitly structured intermediate format (Feldman \& Sanger, 2007).

Text mining techniques are used, for example, for text classification, where textual objects from a universe are assigned to two or more classes (Manning \& Schütze, 1999). Common applications in educational measurement classify students' essays into different grade levels with automated scoring algorithms, e.g., Project Essay Grade (PEG; Page, 2003) and automated scoring of open answer questions, e.g., E-raters (Burstein, 2003). Feature extraction and machine learning are the two essential sections in text classification, playing influential roles in classification efficiency. During feature extraction, textual components are transformed into structured data and labeled with one or more classes. Based on these encoded data, the most discriminative lexical features are extracted by using computational statistic models, such as the chi-square selection algorithm (Oakes, Gaizauskas, Fowkes, Jonsson, \& Beaulieu, 2001) and likelihood ratio functions (Dunning, 1993). In the machine learning section, documents are allocated into the most likely classes by applying machine learning algorithms such as decision trees (DTs), naïve Bayes (NB), support vector machines (SVM), and the $K$ nearest neighbor model $(\mathrm{KNN})$. Although many machine learning classifiers have been tested efficiently in text classification, new alternative models are still being explored to further improve text classification performance and accelerate the speed of word processing (see more in Duda, Hart, \& Stork, 2001; Vapnik, 1998).

This chapter briefly describes the general procedure for supervised text classification where the actual status (label) of the training data has been identified ("supervised"), introduces an effective and much used feature extraction model, i.e., the chi-square selection algorithm, and presents an alternative machine learning algorithm for text classification, named the product score model (PSM). To evaluate the PSM performance, a comparative study was conducted between PSM and two standard classification models, DTs and NB, based on an example application for real textual data. The research questions focus on (a) whether the PSM performs more efficiently in classifying text compared to the standard models, and (b) whether the PSM maintains stable and reliable agreement with the human raters' assessment.

\section{Supervised Text Classification}

Supervised text classification is a commonly used approach for textual categorization, which generally involves two phases, a training phase and a prediction phase (Jurafsky \& Martin, 2009; see Figure 1). 
During training, the most discriminative keywords for determining the class label are extracted. The input for the machine learning algorithm consists of a set of prespecified keywords that may potentially be present in a document and labels classifying each document. The objective of the training phase is to "learn" the relationship between the keywords and the class labels. The prediction phase plays an important role in checking how well the trained classifier model performs on a new dataset. The test set should consist of data that were not used during training. In the testing procedure, the keywords extracted from the training are scanned in each new input. Thus, the words that were systematically recognized are fed into the "trained" classifier model, which predicts the most likely label for each new self-narrative. To ensure proper generalization capabilities for the text classification models, a crossvalidation procedure is generally applied.

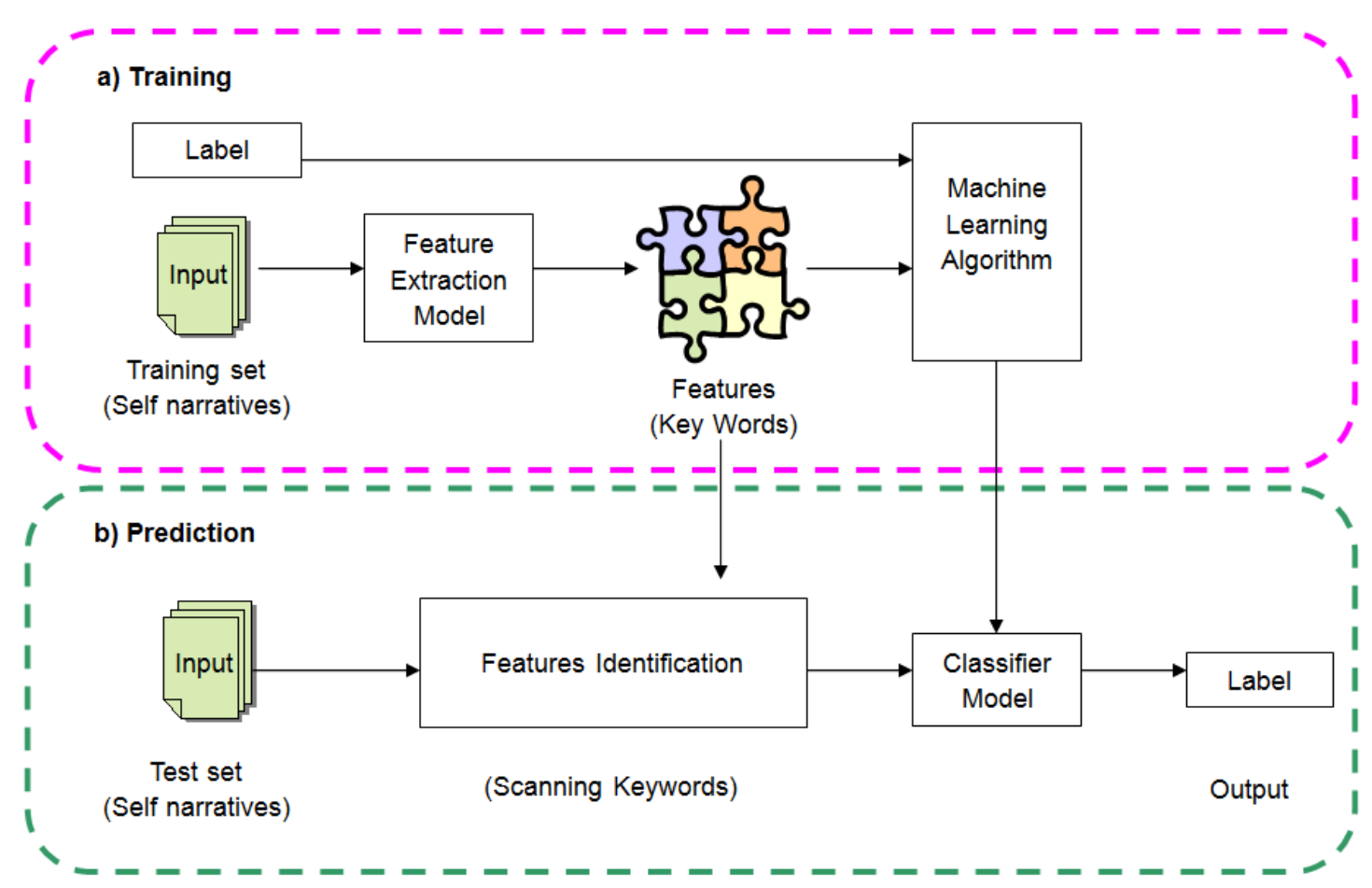

Note: Supervised text classification generally involves two phases, training and prediction. The objective of the training phase is to model (i.e., to learn) the relationship between the keywords and labels. The prediction is used to check how well the trained classifier model performs on a new dataset.

Figure 1 The framework of supervised text classification 
To improve the efficiency of the training and prediction procedure, a preprocessing routine is often implemented. This involves screening digital numbers, deducting noninformative "stop words" (e.g., "I", “to”), common punctuation marks (e.g., “.”, “.”), and frequently used abbreviations (e.g., "isnt", "Im"), and "stemming" the rest of words, for instance, with the Porter algorithm (Porter, 1980) to remove common morphological endings. For example, the terms "nightmares," "nightmaring," and "nightmared," though in variant lexical forms, are normalized in an identical stem "nightmar" by removing the suffixes and linguistic rulebased indicators.

\section{Chi-Square Feature Selection Algorithm}

A classifier extraction can be designed to capture salient words or concepts from texts using a feature selection algorithm that compares the frequency of each word type in the text corpus ${ }^{2}$ of interest to the frequency of that word type in the whole text corpora (Conway, 2010). Forman (2003) reviewed many feature selection methods for text classification, in which the chi-square selection algorithm (Oakes et al., 2001) was recommended for use due to its high effectiveness in finding robust keywords and testing for the similarity between different corpora. Thus, we briefly introduce this algorithm here and then apply it in the example data.

To apply the chi-square algorithm for feature selection, the $N$ word types in the training set are compiled into an $\mathrm{N}$-by-2 table, schematically shown in Table 1 . The two columns correspond to the two corpora, $C_{1}$ and $C_{2}$. Each row corresponds to a particular word $i$. The number of word occurrences in $C_{1}$ and $C_{2}$ is indicated by $n_{i}$ and $m_{i}$, respectively. The sum of the word occurrences in each corpus is defined as the corpus length,

$$
\operatorname{len}\left(C_{1}\right)=\sum_{i=1}^{k} n_{i}, \quad \operatorname{len}\left(C_{2}\right)=\sum_{i=1}^{k} m_{i}
$$


Table 1 Structuralizing Textual Data in a Binary Classification

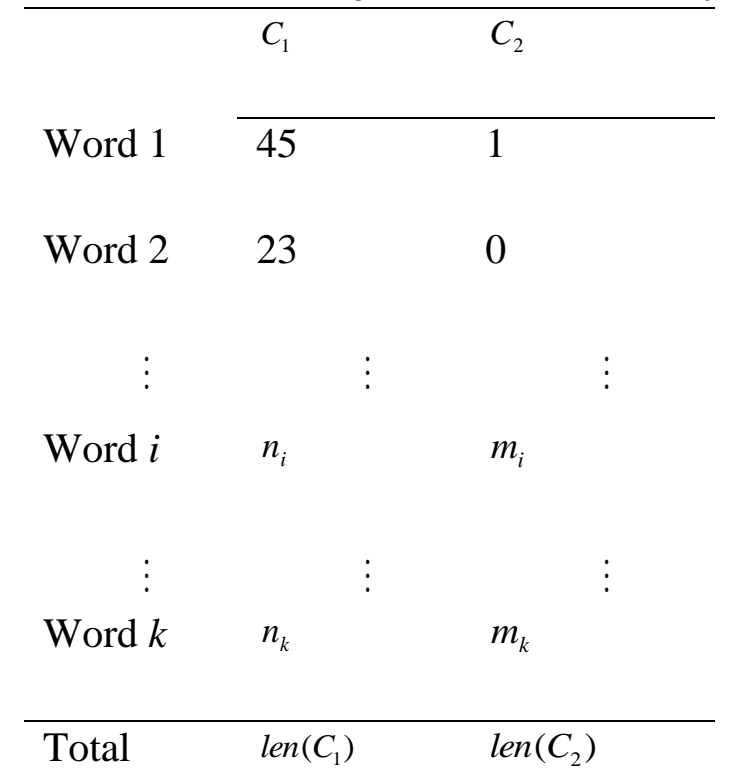

Note: $C$ represents the class label of text corpus, and $n_{i}$ and $m_{i}$ represent the number of occurrences of a word $i$ in two corpora, respectively

Table 2 Confusion Matrix for Word i in the 2-by-2 Chi-Square Score Calculation

$C_{1} \quad C_{2}$

Word $i \quad m_{i}$

$\neg$ Word $i \quad \operatorname{len}\left(C_{1}\right)-n_{i} \quad \operatorname{len}\left(C_{2}\right)-m_{i}$

Note: $C$ represents the class label of text corpus, and $n_{i}$ and $m_{i}$ represent the number of occurrences of a word $i$ in two corpora, respectively

Each word is then compiled into its own 2-by-2 contingency table as shown in Table 2. The values in each cell are called the observed frequencies $\left(O_{i j}\right)$. Using the assumption of independence, the expected frequencies $\left(E_{i j}\right)$ are computed from the marginal probabilities. The chi-square statistic sums the differences between the observed and the expected values in all squares of the table, scaled by the magnitude of the expected values, as the following formula:

$$
X^{2}=\sum_{i, j} \frac{\left(O_{i j}-E_{i j}\right)^{2}}{E_{i j}} .
$$


To ensure the reliability of the calculation, as Manning and Schütze (1999) suggested, in practice features or words that occur fewer than five times are usually eliminated. However, for a small sample, the number of word occurrences could be even lower, perhaps three times. Based on the chi-square scores, all words are ranked in descending order, and those standing at the top are extracted as robust classifiers. ${ }^{3}$ Further, if the ratio $n_{i} / m_{i}$ is larger than the ratio $\operatorname{len}\left(C_{1}\right) / \operatorname{len}\left(C_{2}\right)$, the word is regarded as more typical of corpus $C_{1}$ (as a "positive indicator"); otherwise, it is more typical of corpus $C_{2}$ (as a "negative indicator") (Oakes et al., 2001).

\section{Text Classification Models}

Training text classifiers is the procedure where machines "learn" to automatically recognize complex patterns, to distinguish between exemplars based on their different patterns, and to make intelligent predictions on their class. Among various machine learning algorithms, decision trees (C4.5; Quinlan, 1993) and naïve Bayes are two of the most widely used text classification models (see more algorithms in Kotsiantis, 2007).

\section{Decision Trees}

A decision tree is a well-known machine learning approach to automatically induce classification trees based on training data sets. In the tree structures, leaves represent class labels, and branches represent conjunctions of features that lead to those class labels. The feature that best divides the training data is the root node of the tree. There are numerous methods for finding the feature that best divides the training data such as information gain (Hunt, Marin, \& Stone, 1966) and the Gini index (Breiman, 1984). The objects at each node are split into piles in a way that gives maximum information gain and stopped until they are categorized into a terminate class.

\section{Naive Bayes}

Naive Bayes is a probabilistic classifier applying Bayes's theorem with strong (naive) independence assumptions (Lewis, 1998). It is simple but effective in practice (Hand \& Yu, 2001). In text classification, the basic idea behind NB is to estimate probabilities of categories given a text document by using the joint probabilities of words and categories with the assumption of word independence. Namely,

$$
P(C, \mathbf{w})=\frac{p(C) p\left(w_{1} \mid C\right) p\left(w_{2} \mid C\right) \ldots p\left(w_{k} \mid C\right)}{p\left(w_{1}, \ldots, w_{k}\right)}=\frac{p(C) \prod_{i=1}^{k} p\left(w_{i} \mid C\right)}{p(\mathbf{w})},
$$


where $C$ represents a specific class and $\mathbf{w}$ represents the keyword vectors. $p(C)$ is the prior probability of a certain class, and $p\left(w_{i} \mid C\right)$ is the conditional probability of a word occurs in a certain class. In the binary classification, the two probabilities from categories $C_{1}$ and $C_{2}$ could be simply compared in a ratio $R$. That is,

$$
R=\frac{P\left(C_{1}, \mathbf{w}\right)}{P\left(C_{2}, \mathbf{w}\right)}=\frac{p\left(C_{1}\right) \prod_{i=1}^{k} p\left(w_{i} \mid C_{1}\right)}{p\left(C_{2}\right) \prod_{i=1}^{k} p\left(w_{i} \mid C_{2}\right)} .
$$

If $R>1$, the object is classified in category $C_{1}$; else it is classified in category $C_{2}$.

\section{Product Score Model}

The product score model (He, Veldkamp, \& de Vries, 2012) is an alternative machine learning algorithm, which features in assigning two weights for each keyword (in binary classification) - the probability of the word $i$ occurs in the two separate corpora, $U_{i}$ and $V_{i}-$ to indicate to how much of a degree the word can represent the two classes. The weights are calculated by

$$
\left\{\begin{array}{l}
U_{i}=\left(n_{i}+a\right) / \operatorname{len}\left(C_{1}\right) \\
V_{i}=\left(m_{i}+a\right) / \operatorname{len}\left(C_{2}\right)
\end{array} .\right.
$$

Note that a smoothing constant $a$ (we use $a=0.5$ in this study) is added to the word occurrence in Formula (5) to account for words that do not occur in the training set, but might occur in new texts. (For more on smoothing rules, see Manning \& Schütze, 1999; Jurafsky \& Martin, 2009.)

The name product score comes from a product operation to compute scores for each class, i.e., $S_{1}$ and $S_{2}$, for each input text based on the term weights. That is,

$$
\left\{\begin{array}{l}
S_{1}=P\left(C_{1}\right) \cdot \prod_{i=1}^{k} U_{i}=P\left(C_{1}\right) \cdot \prod_{i=1}^{k}\left[\left(n_{i}+a\right) / \operatorname{len}\left(C_{1}\right)\right] \\
S_{2}=P\left(C_{2}\right) \cdot \prod_{i=1}^{k} V_{i}=P\left(C_{2}\right) \cdot \prod_{i=1}^{k}\left[\left(m_{i}+a\right) / \operatorname{len}\left(C_{2}\right)\right]
\end{array}\right.
$$


where $a$ is a constant, and $P(C)$ is the prior probability for each category given the total corpora. The classification rule is defined as:

$$
\text { choose }\left\{\begin{array}{l}
C=1 \text { if } \log \left(S_{1} / S_{2}\right)>b \\
C=2 \text { else }
\end{array}\right.
$$

where $b$ is a constant. ${ }^{4}$

To avoid mismatches caused by randomness, unclassification rules are also taken into account. As mentioned above, based on the chi-square selection algorithm, the keywords are labeled as two categories, positive indicator and negative indicator. Thus, we define a text as "unclassified" when either one of the following conditions is met: (a) no keywords are found in the text; (b) only one keyword is found in the text; (c) only two keywords are found in the text, and one is labeled as a positive indicator while the other as a negative indicator.

\section{Example Application}

\section{Data}

As part of a larger study exploring the relationship between life narratives and students' personality adaption, 656 life stories were collected from 271 undergraduate students at Northwestern University, in the United States. The classification target was to label the life stories into four categories: redemption (RED), contamination $(\mathrm{CON})$, redemption and contamination (BOTH), and neither redemption nor contamination (NEITHER). In the narrative research in the discipline of personality psychology, redemption and contamination are the two most important sequences for revealing the "change" tendency in people's emotional well-being through writing (McAdams, 2008). In a redemption sequence, a demonstrably "bad" or emotionally negative event or circumstance leads to a happy outcome, whereas in a contamination scene, a good or positive event or state becomes bad or negative. Three experienced experts were invited to label each story based on McAdams's manual coding system (McAdams, 2008). The Kappa agreement among the three human raters was 0.67 .

The label for each story was defined as the decision made by at least two human raters, and was identified as the "standard" for the training process. According to the human raters' assessment, 231 stories were labeled "change" (i.e., redemption or contamination or both), and 425 stories were labeled "no change" (i.e., neither redemption nor contamination). 


\section{Method}

Given concerns about the common feature - "the change" tendency_-in the redemption and contamination sequences, a two-stage classification framework was constructed. On the first stage, all the input was divided into two groups, "change" and "no change." A further detailed classification was conducted at the second stage to categorize the preliminary results as redemption and contamination. To illustrate the application of the text classification models, we focused only on the first stage in the present study. The dataset was randomly split into a training set and a testing set, $70 \%$ and 30\%, respectively. The "stop word list" and the Porter algorithm were used in the preprocessing to deduct the noninformative words and normalize the words into their common lexical forms. The robust classifiers were extracted by using the chi-square selection algorithm. Three machine learning models, DC, NB, and PSM, were applied for a comparative study.

Six performance metrics, accuracy, sensitivity (recall), specificity, positive predictive value (precision) (PPV), negative predict value (NPV), and F1 measure, were used to evaluate the efficiency of the three employed machine learning algorithms. A contingency table was used to perform calculations (see Table 3). All six indicators are defined in definitions (1) through (6), respectively. Accuracy, the main metric used in classification, is the percentage of correctly defined texts. Sensitivity and specificity measure the proportion of actual positives and actual negatives that are correctly identified, respectively. These two indicators do not depend on the prevalence (i.e., proportion of "change" and "no change" texts of the total) in the corpus, and hence are more indicative of real-world performance. The predictive values, PPV and NPV, are estimators of the confidence in predicting correct classification; that is, the higher predictive values, the more reliable the prediction would be. The F1 measure combines the precision and recall in one metric, which is often used in information retrieval to show classification efficiency. This measurement can be interpreted as a weighted average of the precision and recall, where an F1 score reaches its best value at 1 and worst value at 0 .

Further, to check the stability of the three classification models, we explored all the metrics with an increasing number of word classifiers by adding 10 keywords, five from positive classifiers (i.e., "change") and five from negative classifiers (i.e., "no change"), each time. The number of keywords included in the textual assessment ranged from 10 to 2,600. 
Table 3 Contingency Table for Calculating Classification Metrics

\section{True Standard}

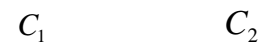

Assigned $C_{1}$

a B

Assigned $C_{2}$

$c$

$d$

Note: $\mathrm{a}$ is a true positive value (TP), $\mathrm{b}$ is a false positive value (FP), c is a false negative value (FN), and $d$ is a true negative value $(\mathrm{TN})$

Accuracy $=\frac{a+d}{a+b+c+d} \quad 1$

Sensitivity $=\frac{a}{a+c}$

Specificit $y=\frac{d}{b+d}$

Positive Predictive Value $(P P V)=\frac{a}{a+b}$

Negative Predictive Value $(N P V)=\frac{d}{5 c+d}$

F1-score $=\frac{2 \times \text { Sensitivity } \times P P V}{\text { Sensitivity }+P P V}$

\section{Notes:}

${ }^{1}$ The stemming algorithm is used to normalize lexical forms of words, which may generate stems without an authentic word meaning, such as "nightmar."

${ }^{2} \mathrm{~A}$ body of texts is usually called a text corpus. The frequency of words within the text corpus can be interpreted in two ways: word token and word type. Word token is defined as individual occurrence of words, i.e., the repetition of words is considered, whereas word type is defined as the occurrence of different words, i.e., excluding repetition of words.

${ }^{3}$ Since we are interested only in ranking the chi-square score for each word to find the optimal classifier, assessing the significance of the chi-square test is not important in this way.

${ }^{4}$ In principle, the scope of threshold $b$ could be set to be infinite. However, in practice, $(-5,+5)$ is recommended as a priori for $b$. 


\section{Results}

Among the top 20 robust positive classifiers (i.e., keywords representing a "change" tendency), the expressions with negative semantics, e.g., "death," “depress," "scare," "lost," "anger," "die," "stop," took a one-third proportion; whereas among the top 20 robust negative classifiers (i.e., keywords representing "no change" tendency), expressions with positive semantics, e.g., "peak," “dance," "high," “promo," "best," “excite," “senior,” accounted for the most, around $35 \%$.

This result implies that people generally describe life in a happy way. The words with negative semantics would be informative for detecting the "change" tendency in the life stories.

The performances of three classification models are shown in Figure 2 with six metrics. Note that the three models resulted in a similar overall accuracy rate of around $70 \%$, although the PSM was a bit superior to the other two, yet not robust. Further, the PSM ranked the highest in the F1 measure, which suggested that this model performed more efficiently than the DT and the NB in the text classification. In the sensitivity analysis, the NB yielded the highest specificity (more than 90\%) but sacrificed too much in sensitivity (around 10\%). The PSM performed worst on specificity (around 75\%) but yielded the best result in sensitivity (around 60\%). The PSM was more sensitive in detecting "change" life stories but a bit less capable of finding "no-change" stories than the other two models. However, among the three models, the PSM was the most balanced between sensitivity and specificity; that is, this model showed relatively satisfactory sensitivity without losing too much specificity. Another noticeable point was that the PSM showed the highest value in the NPV. This implies that we could have the most reliable prediction to deduct "no-change" life stories from the further stage by using the PSM rather than the DT and the NB. In the PPV plot, the NB curve ranked highest but it waved substantially with the increasing number of keywords, whereas the DT and the PSM remained stable throughout the whole processing.

The PSM and DT showed relatively low PPV values (around 60\%), suggesting that the confidence for reliable prediction of "change" life stories was not that strong. However, since at this preliminary stage we targeted discarding the "no-change" life stories from further classification, PPV is less important than NPV in this sense. 

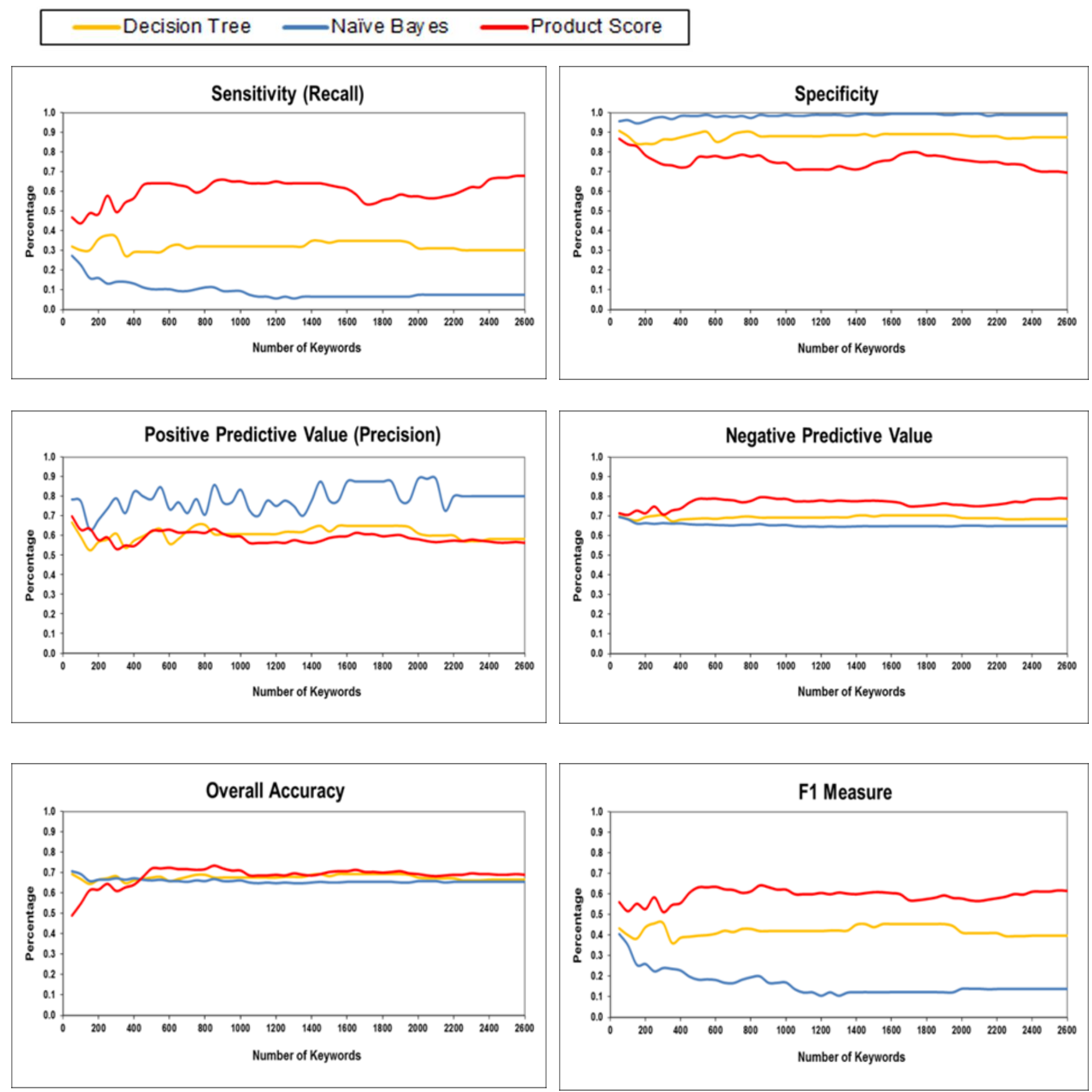

Note: The horizontal axis indicates the number of keywords included in the textual assessment. The text analysis started with 10 keywords with the highest chi-square scores, i.e., five keywords labeled as positive classifiers and five keywords labeled as negative classifiers, and ended with 2600 keywords, i.e., 1300 keywords from either classifier label

Figure 2 Comparisons of text classification models, DT, NB and PSM based on the example application 


\section{Discussion}

The example study demonstrated that the PSM is a promising machine learning algorithm for text (binary) classification. Although the three classification models showed a similar overall accuracy rate, the PSM performed the best in the F1 measure and remained stable as the number of keywords increased, implying better efficiency in text classification and more reliable agreement with the human raters' assessment than the other two standard models. Similar results were found in a recent study by He et al. (2012), where the PSM was validated in text classification for posttraumatic stress disorder (PTSD) patients' self-narratives regarding their stressful events and physical and mental symptoms. Analogous to the example application, the PSM successfully classified the self-narratives written by individuals with PTSD and non-PTSD in high agreement (82\%) with the psychiatrists' diagnoses and presented stable results as the number of keywords increased.

Further, to help practitioners select an optimal algorithm for their own problems, the following pros and cons of each model can be considered and compared. The DT model is one of the most comprehensive models for visually tracking the path in classification. It is easily understood why a decision tree classifies an instance as belonging to a specific class. However, this model may result in low accuracy, especially for a small sample dataset. The DT uses splits based on a single feature at each internal node. Thus, many features are necessary to extract from the training set. Another frequent problem that may occur in applying DT algorithms is the overfitting. The most straightforward way of using them is to preprune the tree by not allowing it to its full size (Kotsiantis, 2007) or establish a nontrivial termination criterion such as a threshold test for the feature quality metric (see more in Elomaa, 1999; Bruha, 2000).

The major advantages of NB are its short computational time for training and its simple form of a product with the assumption of independence among the features. Unfortunately, the assumption of independence among words is not always correct, and thus, the NB is usually less accurate than other more sophisticated learning algorithms. However, the NB is still a very effective model in classification. Domingos and Pazzani (1997) performed a large-scale comparison of the NB with state-of-the-art algorithms, e.g., DT, instance-based learning, and rule induction, on standard benchmark datasets, and found it to be sometimes superior to the other learning schemes, even on datasets with substantial feature dependencies. 
Despite adopting the same assumption of word independence in the NB, the PSM has more flexibility in the model decision threshold. As shown in Formula (7), the decision threshold $b$ could be set as an unfixed constant in practice. For instance, in a clinical setting such as the PTSD screening process, on one hand, psychiatrists may want to exclude people without PTSD from further tests, which needs a relatively higher specificity value.

On the other hand, when psychiatrists focus on treatment for patients with PTSD, a more sensitive result from the text analysis is probably required to detect potential patients as precisely as possible. With the example data in the current study, to yield satisfactory sensitivity in finding the "change" elements in life stories without sacrificing too much specificity, an optimal threshold of PSM log ratio score could be set at $b=-4$. However, since the PSM allocates a set of term weights for each key feature, more time and more storage space are expected in the training and validation process, which might reduce the PSM's effectiveness in a large sample.

In addition to the applications of text classification within the field of psychology and psychiatry, the PSM is also expected to extend its usage in educational measurement. For instance, this model might be used as an alternative approach to classify students' essays into different grade levels, to retrieve information about students' noncognitive skills by analyzing their writing components, e.g., diaries, posts, blogs, and short messages, and further to extract patterns among students' noncognitive skills and their academic grades.

In conclusion, the present study introduced the general procedure of text classification within the framework of text mining techniques and presented an alternative machine learning algorithm, the PSM, for text classification. In the comparative study with two standard models, DT and NB, the PSM was shown to be very promising in text (binary) classification. It might be interesting to extend the PSM into a generalized multiple classification algorithm in future work, and to find out whether and how educational measurement could benefit from this new procedure.

\section{References}

Breiman, L. (1984). Classification and regression trees. Belmont, CA: Wadsworth.

Bruha, I. (2000). From machine learning to knowledge discovery: Survey of preprocessing and postprocessing. Intelligent Data Analysis, 4, 363-374.

Burstein, J. (2003). The E-rater scoring engine: Automated essay scoring. In M. D. Shermis \& J. C. Burstein (Eds.), Automated essay scoring: A cross-disciplinary perspective (pp. 113-121). Mahwah, NJ: Erlbaum. 
Conway, M. (2010). Mining a corpus of biographical texts using keywords. Literary and Linguistic Computing, 25(1), 23-35.

Domingos, P., \& Pazzani, M. (1997). On the optimality of the simple Bayesian classifier under zero-one loss. Machine Learning, 29(2-3), 103-130.

Duda, R. O., Hart, P. E., \& Stork, D. G. (2001). Pattern classification. New York, NY: Wiley.

Dunning, T. (1993). Accurate methods for the statistics of surprise and coincidence. Computational Linguistics, 19, 61-74.

Elomaa, T. (1999). The biases of decision tree pruning strategies. Advances in Intelligent Data Analysis Proceedings, 1642, 63-74.

Feldman, R., \& Sanger, J. (2007). The text mining handbook: Advanced approaches in analyzing unstructured data. Cambridge, England: Cambridge University Press.

Forman, G. (2003). An extensive empirical study of feature selection metrics for text classification. Journal of Machine Learning Research, 3, 1289-1305.

Hand, D. J., \& Yu, K. M. (2001). Idiot's Bayes - Not so stupid after all? International Statistical Review, 69(3), 385-398.

He, Q., Veldkamp, B. P., \& de Vries, T. (2012). Screening for posttraumatic stress disorder using verbal features in self-narratives: A text mining approach. Psychiatry Research. doi: 10.1016/j.psychres.2012.01.032

Hunt, E. B., Marin, J., \& Stone, P. J. (1966). Experiments in induction. New York, NY: Academic Press.

Jurafsky, D., \& Martin, J. H. (2009). Speech and language processing: An introduction to natural language processing, computational linguistics, and speech recognition. Upper Saddle River, NJ: Pearson Prentice Hall.

Kotsiantis, S. B. (2007). Supervised machine learning: A review of classification techniques. Informatica, 31, 249-268.

Lewis, D. (1998). Naive (Bayes) at forty: The independence assumption in information retrieval. In C. Nedellec \& C. Rouveirol (Eds.), Machine learning: ECML-98, Proceedings from the 10th European Conference on Machine Learning, Chemnitz, Germany (pp. 4-15). New York, NY: Springer.

Manning, C. D., \& Schütze, H. (1999). Foundations of statistical natural language processing. Cambridge, MA: MIT Press.

McAdams, D. P. (2008). Personal narratives and the life story. In O. P. John, R. W. Robins, \& L. A. Pervin (Eds.), Handbook of personality: Theory and research (pp. 242-264). New York, NY: Guilford. 
Oakes, M., Gaizauskas, R., Fowkes, H., Jonsson, W. A. V., \& Beaulieu, M. (2001). A method based on chi-square test for document classification. In: Proceedings of the 24th Annual International ACM SIGIR Conference on Research and Development in Information Retrieval (pp. 440-441). New York, NY: ACM.

Page, E. B. (2003). Project Essay Grade: PEG. In M. D. Shermis \& J. C. Burstein (Eds.), Automated essay scoring: A cross-disciplinary perspective (pp. 43-54). Mahwah, NJ: Erlbaum.

Porter, M. F. (1980). An Algorithm for Suffix Stripping. Program-Automated Library and Information Systems, 14(3), 130-137.

Quinlan, J. R. (1993). C4.5: Programs for machine learning. San Francisco, CA: Morgan Kaufmann.

Vapnik, V. N. (1998). Statistical learning theory. New York, NY: Wiley. 


\title{
Chapter 6
}

\section{Minimizing the Testlet Effect: Identifying Critical Testlet Features by Means of Tree-Based Regression}

\author{
Muirne C.S. Paap and Bernard P. Veldkamp
}

\begin{abstract}
Standardized tests often group items around a common stimulus. Such groupings of items are called testlets. The potential dependency among items within a testlet is generally ignored in practice, even though a basic assumption of item response theory (IRT) is that individual items are independent of one another. A technique called tree-based regression (TBR) was applied to identify key features of stimuli that could properly predict the dependence structure of testlet data. Knowledge about these features might help to develop item sets with small testlet effects. This study illustrates the merits of TBR in the analysis of test data.
\end{abstract}

Keywords: testlets, testlet effects, testlet response theory, tree-based regression

\section{Introduction}

Standardized educational tests (which are often high-stakes tests) commonly contain sets of items grouped around a common stimulus, for example, a text passage, graph, table, or multimedia fragment, creating a dependence structure among items belonging to the same stimulus. Such groups of items are generally referred to as item sets or testlets (Wainer \& Kiely, 1987), and this kind of dependence has been referred to as passage dependence (Yen, 1993). Testlets are popular for several reasons, including time efficiency and cost constraints, reducing the effects of context in adaptive testing, and circumventing concerns that a single independent test might be too atomistic in nature (measuring a concept that is very specific or narrow) (Wainer, Bradlow, \& Du, 2000). In the Netherlands, testlets are, for example, used in the final examinations at the end of secondary education and in the "Cito test" (van Boxtel, Engelen, \& de Wijs, 2011).

In most high-stakes tests, item response theory (IRT) models (Lord, 1980) are applied to relate the probability of a correct item response to the ability level of the candidate. A basic assumption underlying these models is that the observed responses to any pair of items are independent of each other given an individual's score on the latent variable (local independence, or LID). 
However, for pairs of items grouped around the same testlet, responses might also depend on the common stimulus. Examinees might misread or misinterpret the stimulus, not like the topic, have particular expertise on the subject matter addressed by the stimulus, and so on.

In certain situations, the testlet structure could be accounted for by applying a polytomous IRT model, like the partial credit model, at testlet level, where the sumscore of the items in the testlets would function as the score on this polytomous item (e.g., Thissen, Steinberg, \& Mooney, 1989; Verhelst \& Verstralen, 2008). This polytomous approach to testlets would not result in any violations of local independence, and standardized software could be applied to estimate the models. However, there are some drawbacks. Until now, this approach has only been proposed for situations where the items within a testlet adhere to the very strict Rasch model. Furthermore, in calculating sumscores, the exchangeability of items is assumed, which may not be realistic in practice. Moreover, a guessing parameter at the item level cannot be taken into account. Alternatively, an approach can be used that accounts for the multilevel structure (items within testlets). Bradlow, Wainer, and Wang (1999) proposed to model the testlet effect by introducing a new parameter to the IRT models that accounts for the random effect of a person on items that belong to the same testlet, in order to adjust for the nested structure. This parameter, $\gamma_{n t}$, is referred to as the testlet effect for person $n$ on testlet $t$. It represents a random effect that exerts its influence through its variance: the larger the variance $\sigma_{1 t}^{2}$, the larger the amount of local dependence (LD) between the items $j$ within the testlet $d$ (Wainer \& Wang, 2000).

Although several procedures for estimating testlet response models have been developed and applications of testlet response theory (TRT) have been studied (Glas, Wainer, \& Bradlow, 2000; Wainer, Bradlow, \& Wang, 2007), the dependency is often ignored in practice, and standard IRT models are used instead. The reason is obvious: assuming that LID holds, allows the use of simpler and well-known IRT analyses using easily accessible software. However, ignoring LD may lead to underestimation of the standard error of the ability estimates, as well as bias in the estimated item difficulty and discrimination parameter if the testlet effect is of a medium to large size (Wainer \& Wang, 2000; Yen, 1993).

One way to approach this issue is to design testlets that show a small testlet effect. In a simulation study, Glas et al. (2000) investigated what the effect would be on the accuracy of item calibration if the testlet structure were to be ignored. 
Their data-set was generated using the 3PL model and the following structure: $a_{i} \sim U(0.8,1.2), b_{i} \sim U(-1,1), c_{i}=0.25$, and $\theta \sim N(0,1)$. They compared the outcomes for the two values of $\sigma_{1 t}^{2}: 0.25$ and 1.00. It should be noted that values of 1.00 or larger are often found in real data-sets. Their findings showed that the $\sigma_{1 t}^{2}$ value of 0.25 resulted in negligible bias in item parameter estimates, whereas moderate effects were found for the $\sigma_{1 t}^{2}$ value of 1.00 (Glas et al., 2000). Thus, if the testlet effects are small, the LD violation would be in an acceptable range, and models such as the $2 \mathrm{PL}$ or $3 \mathrm{PL}$ could be used without sacrificing the quality of the parameter estimation. A requirement for designing such testlets, however, is knowing which testlet characteristics are related to the testlet effect size.

\section{Predicting Testlet Effects}

In a recent study (Paap, He, \& Veldkamp, submitted), which will be referred to here as "study 1," we used tree-based regression (TBR) to identify the key features of the stimuli that can predict the testlet effect in a standardized test measuring analytical reasoning. TBR is a popular method in the field of data mining, but it is becoming more popular in other fields as well, including educational measurement (e.g., Gao \& Rogers, 2011). Like in other forms of regression analysis, TBR involves a set of independent variables and one or more dependent variables. Independent variables can be nominal, ordinal, or interval variables. A dependent variable is a continuous variable; if it is categorical in nature, a classification tree is generated. Independent variables can enter the tree more than once. Among TBR's advantages are its nonparametric nature, ease of interpretation, and flexibility in dealing with high-order interaction terms. An example of such a high-order interaction can be found in Figure 1: nodes 11 and 12, which are positioned in the right branch. These two nodes are the result of an interaction between four independent variables!

TBR can be used to divide the set of testlets iteratively in increasingly homogeneous subsets (so-called "nodes"). At each stage of the analysis, the testlet feature with the largest influence on the dependent variable is identified by using a recursive partitioning algorithm called the "classification and regression tree" (CART) (Breiman, Friedman, Olshen, \& Stone, 1984). The CART algorithm starts by growing a large initial tree which overfits the data so as to not miss any important information.

In the next step, the tree is "pruned": a nested sequence of subtrees is obtained and, subsequently, one of them is selected based on pre-defined criteria. Typically, the final step consists of cross-validating the tree to determine the quality of the final model further. 
Since we had a relatively small data-set $(100 \text { testlets })^{1}$ in our study, the cross-validation resulted in trees with little explained variance, and there was a substantial effect of the random splitting of the data-set on the findings. Therefore, we chose not to use crossvalidation in our study.

The dependent variable in our TBR is the standard deviation of the testlet parameter, denoted as $\sigma_{1 t}$. Note that we deliberately chose to use $\sigma_{1 t}$ as opposed to $\sigma_{1 t}^{2}$ in our model, since $\sigma_{1 t}$ capitalizes on the difference between testlets and is thus more informative in this setting. We estimated the testlet effect using a three-parameter normal ogive (3PNO) model, which is highly similar to the well-known 3PL model. The responses were coded as $Y_{n i}=1$ for a correct response and $Y_{n i}=0$ for an incorrect response. The probability of a correct response is given by

$$
P\left(Y_{n i}=1\right)=c_{i}+\left(1-c_{i}\right) \Phi\left(a_{i} \theta_{n}+b_{i}+\gamma_{n t(i)}\right)
$$

where $\Phi($.$) is the probability mass under the standard normal density, and c_{i}$ is the guessing parameter of item $i . \gamma_{n t(i)}$ has a normal distribution; that is,

$$
\gamma_{n t} \sim N\left(0, \sigma_{1 t}^{2}\right)
$$

The parameters were estimated in a fully Bayesian approach using an MCMC computation method. For details, see Glas (2012). Note that the model fit of (1) will be investigated in a future study. The average testlet effect estimated with the 3PNO equaled $0.71(\mathrm{SD}=0.16)$. It should be noted that a value of $\sigma_{1 \mathrm{t}}$ smaller than 0.50 has been shown to have a negligible effect on parameter estimates, whereas an effect near the size of 1.00 has a more substantial influence (Glas et al., 2000).

\footnotetext{
${ }^{1}$ Each respondent was presented with four out of 100 testlets; the four testlets were comprised of around 26 items each.
} 


\section{Identifying Testlet Characteristics}

The features used as independent variables in our study can be divided into four categories:

(1) variables describing the logical structure of the stimuli, (2) variables describing the themes contained in the stimuli, (3) surface linguistic variables, and (4) aggregated item characteristics. Two raters independently coded the variables in categories 1 and 2 . In the case of discordant scorings, a consensus was reached through discussion; a discussion log was kept for these stimuli. The surface linguistic features were generated by using the specialized textmining software Python (Python Software Foundation, 2009). The aggregated item characteristics were computed by averaging the attributes over all of the items in a testlet. In total, 22 independent variables were generated.

\section{Study 1: Prediction Based on Testlet Features only}

In our first study, we did not include information about the items in our prediction model. A two-step procedure was applied to build the prediction model. First, separate models were evaluated for each variable category (structure, theme, linguistic). The variables that were selected by the algorithm were then retained for each category, and subsequently all of the variables belonging to one of the other categories were added to the selected variables to see if any of them would be selected in the regression tree. In the next step, all of the variables that were not selected by the CART algorithm were removed from the list of independent variables and the variables of the remaining category were added. We then removed the variables that were not selected from the independent variable list again. In the case of competing models, the final model was selected based on the amount of the explained variance and the greatest number of splits resulting in a large difference in the mean testlet $S D$ for the resulting nodes.

\section{Summary of Results}

Four independent variables were selected for the final prediction model: the percentage of "if" clauses, the predicate propositional density (the number of verbs divided by the total number of words, excluding punctuation), theme/topic, and the number of entities (entities are defined as the units in the stimulus that had to be assigned to positions). The latter two variables entered the tree at several splits. The total explained variance equaled $37.5 \%$. The final tree consisted of 16 nodes. For every node, the mean value of $\sigma_{1 \mathrm{t}}$ was larger than 0.50 . For 6 nodes, the value of $\sigma_{1 \mathrm{t}}$ exceeded 0.75 . For all 6 nodes with a medium-large testlet effect, the percentage of "if" clauses was smaller or equal to $31 \%$. 
The largest testlet effects were found for the stimuli with a predicate propositional density of 0.098 or larger: 0.898 for stimuli with more than 10 entities and 0.980 for stimuli with 4 entities or fewer. For stimuli with a predicate propositional density smaller than 0.098 , the largest testlet effect was found for the stimuli with the theme/topic that was either business, education, transport, or nature related, which had 5 entities or less, and a predicate propositional density between 0.071-0.097.

\section{Study 2: Including Average Item Difficulty}

Since a testlet effect is an additional source of variance in an item response function, the question arises whether attributes of items belonging to the testlet can be used to predict the testlet effect. In study 1, the focus was only on stimulus attributes. In this second study, aggregated item attributes will be included as well. Several interesting questions have to be answered, including whether there is a relationship between the average item difficulty in a testlet and the size of the testlet effect, whether characteristics of the testlet are related to average item difficulty, and whether there is an influence of item characteristics and the testlet location on the testlet effect. We made a first step towards illuminating these issues by investigating the relationship between average item difficulty within a testlet and the testlet effect size. We did this by adding the average item difficulty per testlet to the TBR model described in the previous study. The same two-step procedure for building the model was applied. The only difference was that besides the structure, theme, and linguistic variables, a fourth category of independent variables was added to the model.

\section{Summary of Results}

The resulting tree can be found in Figure 1. The total variance explained for this model was $41.4 \%$, which implies that adding average difficulty as an independent variable improved the model. 


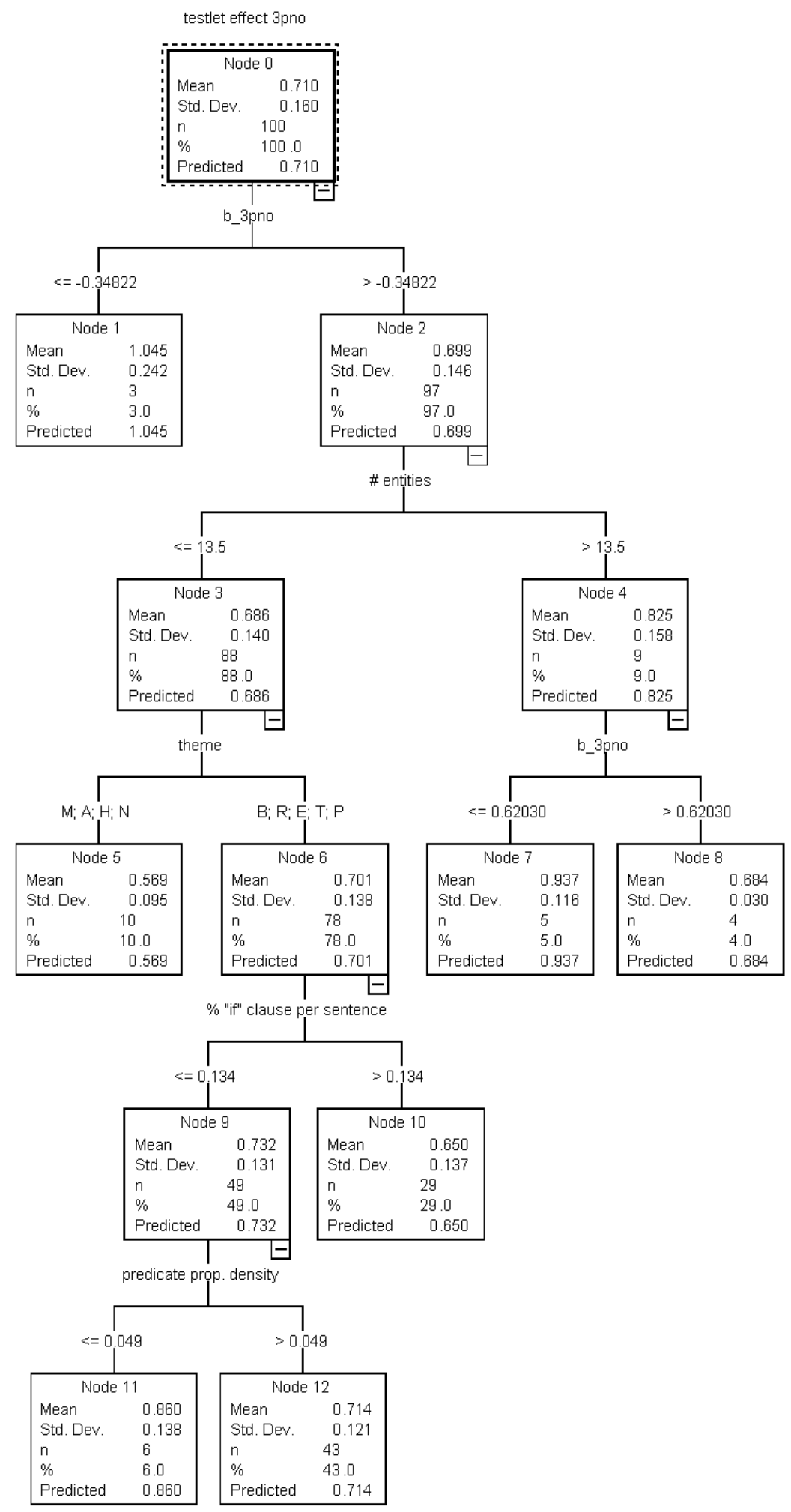

Figure 1 Regression tree based on the final model in study 2 with the 3PNO-based testlet effect as a dependent 
When comparing study 2's model depicted in Figure 1 to the 3PNO-based model described in study 1 , there are several important similarities. First, all of the variables that were contained in the tree described in study 1 were retained in the new model (Figure 1). Also, both models suggest that a large number of entities is associated with a larger testlet effect, and in a subset of testlets a low predicational propositional density score is associated with a larger testlet effect. However, it is important to note that the average item difficulty is chosen for the first split in the newer model, indicating its relative importance.

It can be seen that testlets containing easy items have a larger testlet effect. Furthermore, testlets with an average item difficulty between -0.35 and 0.62 that also contain 14 entities or more are also associated with a high testlet effect. Finally, testlets with an average item difficulty larger than $-0.35 ; 13$ entities or fewer; with a theme related to business, recreation, education, transport, or intrapersonal relationships/family; containing $13.4 \%$ or less "if" clauses; and that had a propositional density score of 0.049 or smaller also showed a larger testlet effect.

\section{Conclusion}

Our findings indicate that, for most testlets, testlet characteristics are associated with the size of the testlet effect, even when the average item difficulty has been accounted for. Three exceptions were found, all testlets with a relatively low average item difficulty. If these findings can be replicated, they may indicate that if testlets predominantly contain easy items, testlet characteristics are either of less importance to the size of the testlet effect or show considerable overlap with the information provided by the average item difficulty. In order to unravel this issue, we will have to explore the relationship between testlet characteristics (as independent variables) and average item difficulty per testlet (dependent variable) in a future study. In addition, other aggregated item variables might have to be added to the model to explore the relationship between item attributes and testlet effects more extensively.

In summary, we found evidence in our study for stimulus-related variables being associated with the size of the testlet effect. Our findings can be used in item construction, and the analyses we applied can be used as an example for others who construct and analyze similar data-sets to ours. However, a little more research is needed before solid "testlet construction rules" can be formulated. 


\section{Acknowledgement}

This study received funding from the Law School Admission Council (LSAC). The opinions and conclusions contained in this report are those of the author and do not necessarily reflect the position or policy of LSAC.

\section{References}

Bradlow, E. T., Wainer, H., \& Wang, X. H. (1999). A Bayesian random effects model for testlets. Psychometrika, 64(2), 153-168.

Breiman, L., Friedman, J. H., Olshen, R. A., \& Stone, C. J. (1984). Classification and regression trees. Belmont, CA: Wadsworth International Group.

Gao, L., \& Rogers, W. T. (2011). Use of tree-based regression in the analyses of L2 reading test items. Language Testing, 28(1), 77-104. doi: 10.1177/0265532210364380

Glas, C. A. W. (2012). Estimating and testing the extended testlet model. LSAC Research Report Series. Newtown, PA: Law School Admission Council.

Glas, C. A. W., Wainer, H., \& Bradlow, E. T. (2000). MML and EAP estimation in testletbased adaptive testing. In W. J. van der Linden \& C. A. W. Glas (Eds.), Computer Adaptive Testing: Theory and Practice (pp. 271-288). Dordrecht, Netherlands: Kluwer.

Lord, F. M. (1980). Applications of item response theory to practical testing problems. Hillsdale, NJ: Erlbaum.

Paap, M. C. S., He, Q., \& Veldkamp, B. P. (submitted). Identifying critical testlet features using tree-based regression: An illustration with the analytical reasoning section of the LSAT.

Thissen, D., Steinberg, L., \& Mooney, J. A. (1989). Trace lines for testlets: A use of multiplecategorical-response models. Journal of Educational Measurement, 26(3), 247-260. doi: 10.1111/j.1745-3984.1989.tb00331.x

van Boxtel, H., Engelen, R., \& de Wijs, A. (2011). Wetenschappelijke verantwoording van de Eindtoets 2010. Arnhem: Cito.

Verhelst, N. D., \& Verstralen, H. H. F. M. (2008). Some considerations on the partial credit model. Psicologica, 29, 229-254. 
Wainer, H., Bradlow, E. T., \& Du, Z. (2000). Testlet response theory: An analog for the 3PL model useful in testlet-based adaptive testing. In W. J. van der Linden \& C. A. W. Glas (Eds.), Computerized adaptive testing: Theory and practise (pp. 245-270). Dordrecht, Netherlands: Kluwer.

Wainer, H., Bradlow, E. T., \& Wang, X. (2007). Testlet response theory and its applications. New York: Cambridge University Press.

Wainer, H., \& Kiely, G. (1987). Item clusters and computerized adaptive testing: A case for testlets. Journal of Educational Measurement, 24, 185-202.

Wainer, H., \& Wang, X. (2000). Using a new statistical model for testlets to score TOEFL. Journal of Educational Measurement, 37(3), 203-220.

Yen, W. M. (1993). Scaling performance assessments: Strategies for managing local item dependence. Journal of Educational Measurement, 30(3), 187-213. 


\title{
Chapter 7
}

\section{Mixed Methods: Using a Combination of Techniques to Assess Writing Ability}

\author{
Hiske Feenstra
}

\begin{abstract}
A productive ability such as writing can be assessed only through a candidate's performance on a task, giving rise to concerns about the reliability and validity of writing assessments. In this chapter, it is argued that a combination of different techniques can help improve the quality of an evaluation of writing ability. First, an indirect test can be applied to reliably assess specific components of the writing process (e.g., revision), adding to the validity of the assessment. Furthermore, an analytic rating procedure accompanied by anchor essays allows raters to reliably evaluate the content and overall structure of written pieces. And last, automated scoring techniques can be used to objectively score text features considered important to text quality. Combining these methods provides an evaluation that is solid and informative.
\end{abstract}

Keywords: writing ability, indirect measurement, anchor essays, automated scoring

\section{Introduction}

Measuring a productive language skill such as writing is notoriously complex. Candidates' writing ability is usually assessed through written products demonstrating their performance on a writing task. As illustrated in several studies over time (Breland, Camp, Jones, Morris, \& Rock, 1987; Godshalk, Swineford, \& Coffman, 1966; Knoch, 2011; Weigle, 2002), the reliability and validity of writing assessments are often questioned. For instance, raters tend to disagree on the quality of the same piece of writing, which impairs reliability, and the discussion of the authenticity of writing assessments is a typical validity issue.

However, techniques such as indirect measurement and the evaluation of essays using an analytic rating procedure accompanied by automated scoring techniques can account for a valid and reliable assessment of specific aspects of the writing process and its end result: a written product. Therefore, a clever mix of assessment techniques can provide for a sound and informative evaluation of writing ability, as is argued in the last paragraph of this chapter. 


\section{Indirect Assessment of Writing}

To overcome these issues, indirect writing tests were developed in the 1960s as an alternative for retrieving information on a candidate's writing ability. These tests are aimed at eliminating rater effects by offering objective tests on components of writing ability, such as spelling or grammatical fluency. Since indirect tests rely on the assumption that writing ability can be deducted via other skills, most research has focused on the correlation between test scores on indirect and direct measures of writing, stating that a high correlation between the two scores validates the use of an indirect instead of a direct measure. Table 1 summarizes a sample of these studies.

Table 1 A Sample of Previous Studies on the Validity of Indirect Writing Assessments

\begin{tabular}{llll}
\hline Study (year) & Age of & Number of & Correlation direct and \\
& pupils & pupils & indirect measure \\
\hline Godshalk et al. (1966) & $16-17$ & 646 & $0.71-0.77$ \\
Wesdorp (1974) & 12 & 213 & $0.67-0.68$ \\
Breland and Gaynor (1979) & 18 & $234-926$ & $0.56-.074$ \\
Breland et al. (1987) & $>18$ & 267 & $0.56-0.66$ \\
\hline
\end{tabular}

Nevertheless, instead of considering indirect tests as substitutes for active writing tasks, perhaps a more valid application for these objective tests is to use them to evaluate different aspects of the writing process. In the 1980s, studies on cognitive writing processes changed the focus for research on writing. Nowadays, writing is no longer considered a single action, but rather as a complicated process in which different components interact. One of the most popular models for the writing process, presupposing interaction among the task environment, long-term memory, and working memory, is shown in Figure 1 (Flower \& Hayes, 1981). 


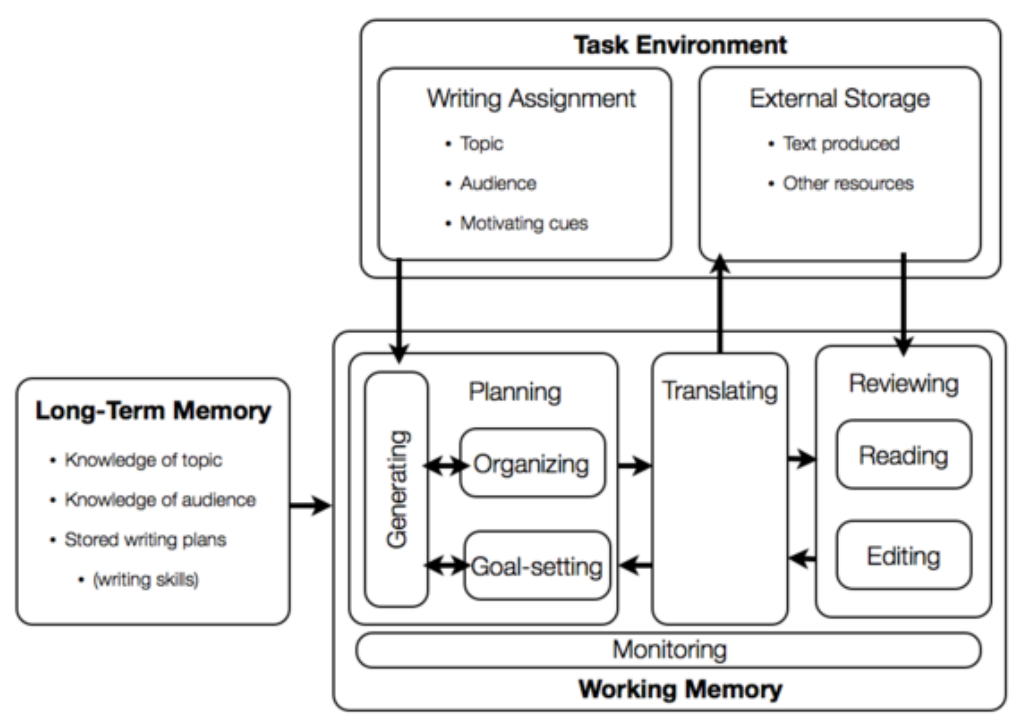

Figure 1 Model of the cognitive writing process by Flower and Hayes (1981)

\section{Evaluation of a New Format for Revision Tests}

A popular form of an indirect writing test is the revision test, where pupils are asked to correct a text supposedly written by a peer. When mapping indirect writing tests to the model composed by Flower and Hayes, this test assesses the part of the writing process referred to as reviewing, where the writer reads and edits his or her text. Feenstra and Heesters (2011a, 2011b) developed a new version of this test as a pilot, changing the multiple-choice format into a semi-open-ended version. In this new format, pupils are asked to actively revise a peerwritten text by deleting or adding words, changing tenses, correcting congruence, et cetera. The sentences to be corrected (i.e. containing errors) were indicated by underlining. Table 2 lists the various options for correction. Since its format is more productive and less directive than the original multiple-choice version of the test, the adapted test is thought to be a more natural representation of reviewing a text.

Table 2 Correction Options in Revision Test

Afgelopen zaterdag ging ik naar mijn oma gegaan. Deleting

zijn

Mijn hobby's is tekenen, judo en gamen.

Als ik vrij

naar

We gingen eerst buiten. Daarna maakten we teams.
Switching

\section{Correcting}

\section{Adding}


To evaluate the new format, both versions of the test (old and new) were incorporated in an incomplete test design. A representative sample of 80 primary schools participated in the study, resulting in a sample of 1,600 pupils. Table 3 reports the results of the pilot study, comparing the test characteristics of the semi-open-ended test version to those of the multiplechoice version.

Table 3 Results on Pilot Study Semi-Open-Ended Writing Test

\begin{tabular}{lll}
\hline & Old & New \\
\hline Reliability & $0.80^{\mathrm{a}}$ & $0.83^{\mathrm{a}}$ \\
Difficulty ( $p$ value) & 0.73 & 0.62 \\
Discriminating power & 3.19 & 2.53
\end{tabular}

Note: ${ }^{\text {a}}$ Estimate for 50 -item test using the Spearman-Brown formula.

Given the above, a semi-open-ended indirect writing test appears to be a reliable tool for assessing specific components of the writing process such as reviewing. Except for items on revision skills, it might also be possible to construct item formats with which other aspects of the writing process can be assessed. Paired with a writing assignment, an indirect writing test can therefore be a useful addition to a valid and reliable assessment of writing.

\section{The Use of Anchor Essays}

When assessing writing via a writing assignment, several different rating procedures are available to evaluate the essays. The most commonly used procedure in classroom assessment is holistic scoring, in which raters assign a score to an essay based on their overall impression of the writing performance (van den Bergh, 1990; Weigle, 2002). A more condensed form of this rating procedure is the primary trait approach. The focus in this method is merely the extent to which the essay reaches its communicative goal (Lloyd-Jones, 1977; van Gelderen, Oostdam, \& van Schooten, 2010). Since raters are asked only to give one overall evaluation, both methods demand relatively little time and effort. As a result, however, these methods do not provide many details on the ability being measured.

Within an analytical rating procedure, different aspects of the writing product are evaluated, enabling a detailed report on writing ability. This analytical method was used in the Dutch National Assessment in Education, where a group of raters used an analytical rating scheme, assessing different aspects of writing (Krom et al., 2004). 
One of the objectives of the analytic evaluation is to alleviate the task of raters by having them answer simple yes/no questions on features of the essay. Consequently, raters only have to identify certain features of the text (scoring), while the actual assigning of values (grading) is done within the data analyses. Because of the relatively simple task for the raters, it was believed that this method would provide high rater agreement. However, analyses show that the inter-rater reliability was rather low for some of the aspects (Krom et al., 2004).

\section{Adjusting the Rating Procedure}

A writing assessment consists of numerous elements, all of them possible sources of construct-irrelevant variance (Messick, 1989): for example, writing task, rating procedure, and rater characteristics. Although recognized, not all of these sources can be eliminated easily. For example, task effects can be ruled out by dramatically increasing the number of tasks given to a student, and rater effects by increasing the number of raters per essay. However, these methods are generally considered unsuitable, given the extra time and effort they would require of both students and raters. Therefore, most studies focus on altering the rating procedure to improve the reliability and validity of a writing assessment.

To achieve high reliability, raters should agree to a great extent on the scores assigned to essays. Providing the raters with an empirically constructed reference, or benchmark, that they can use to compare the quality of the writing products to be assessed could therefore prove to be beneficial for the agreement between raters, and thus have a positive influence on the rater reliability and validity of the writing scores. An empirical way of providing such a reference is constructing a rating scale illustrated with several examples of writing products, each representing a specific score point. The exemplars are taken from a sample of essays evaluated by multiple assessors and vary from a poor performance on one end of the scale to an excellent performance on the other end of the scale.

Van den Bergh and Rijlaarsdam (1986) developed a method to construct such a rating scale. The authors described all the steps needed to create rating scales for different aspects of writing. According to van den Bergh and Rijlaarsdam, using a rating scale with anchor essays has two main advantages over the use of an analytic rating procedure.

First, the exemplars on the rating scale serve as reference points, supporting the raters in their rating task and reducing instability in their rating. Moreover, using a fixed standard allows scores to be compared between pupils and classes or scores to be monitored over time. In the context of a national assessment, anchor essays can be particularly useful for illustrating different levels of achievement. 
In fact, anchor essays were used in earlier cycles of the national assessment for writing (Sijtstra, 1997; Zwarts, 1990), but were eventually replaced in the next cycle owing to their complicated scoring instructions.

\section{Evaluating a Rating Scale with Anchor Essays}

Feenstra (2010b) reported on the use of a rating with anchors essays to improve the inter-rater reliability. In this study, three different essay tasks were selected from the pool of tasks in the Dutch national assessment, covering a broad scope of text goals and text genres. Five Dutch primary schools representing different regions, school sizes, and denominations volunteered to participate in the study. A total of 584 pupils, age 8 to 12 , participated. In total, 1,476 essays were collected. All essays were digitalized (i.e., retyped, maintaining layout, typos, and punctuation) to facilitate reproduction and distribution. Moreover, handwriting quality can influence the assessment of other aspects of text quality (De Glopper, 1988). Presenting the essays in typescript eliminates this unwanted effect. As in the previous cycles of the national assessment, three aspects of writing were to be rated, as shown in Table 4 .

Table 4 Categorization of Writing Aspects Used within the Study

Aspect Description

Content Essential content elements, focus on text goal and public

Structure Composition, layout, coherence, cohesion

Correctness Syntax, spelling, punctuation

The procedure described by van den Bergh and Rijlaarsdam (1986) was adopted to compose a rating scale with anchor essays for each aspect per task (Feenstra, 2010a), the result being a rating scale with three anchor essays representing specific ability levels (Figure $1)$.

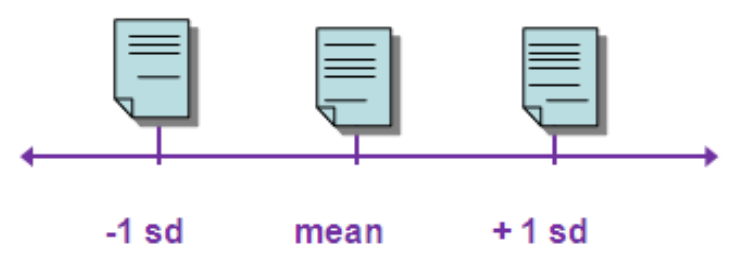

Figure 2 A rating scale with three exemplars 
To select the anchor essays, four expert raters first agreed upon the average essay and then evaluated a sample of essays. The anchor essays for each score point were then selected based on their empirically defined value as an exemplar essay: agreement among the four different raters.

A total of 26 raters scored a sample of 150 essays in an incomplete design, to evaluate the quality and usefulness of the new rating procedure compared to the existing method. Each rater was assigned to one of two conditions, where condition 1 represented the existing analytical rating procedure, and condition 2 represented the adjusted version of the original procedure, consisting of the analytical scale plus the additional rating scale with anchor essays. Each essay was scored in both conditions, by a minimum of two out of the 13 raters assigned to each condition. In Table 5, reliability scores are presented per aspect.

Table 5 Inter-Rater Agreement (Gower's Coefficient) for All Raters

\begin{tabular}{lll}
\hline Aspect & Condition 1 & Condition 2 \\
& Analytical & analytical + anchors \\
\hline Content & .85 & .84 \\
Structure & .76 & $.81 *$ \\
Correctness & .76 & .77 \\
\hline
\end{tabular}

As shown in Table 5, the aspects Structure and Correctness seem to generally benefit from the addition of anchor essays to an analytic rating scale. However, the improvement in inter-rater reliability is modest and significant only for the aspect Structure.

\section{On the Use of Anchor Essays for Different Aspects of Writing}

Text structure was found to be the only aspect for which the use of anchor essays significantly improved reliability. It might well be that for this aspect in particular having a complete essay as a reference for scoring is beneficial. Apart from the structure within sentences, text structure can be evaluated only by considering the text as a whole, which is encouraged by comparing essays to an anchor. For example, when evaluating a letter, the layout and formal structure of the text are important features that should be present not only in one or two parts of the text. Instead, they should form the basis of the text structure. 
An aspect such as Content, however, is more or less locally assessed within a text and less dependent on the overall text quality. Different content elements are detected in the text and scored accordingly: the higher the number of elements that are present, the higher the score. This could be the reason that this aspect did not benefit from the comparison to anchor essays when assessing it. To assess this particular aspect, a detailed analytical procedure seems to be the best option.

In a way, the same holds for the aspect Correctness. This aspect actually requires the impression of the whole text to be taken into account, but as with Content, the elements diminishing the correctness can be more or less counted individually. Although this might sound straightforward, raters tend to disagree relatively strong when scoring this aspect. Apparently, differences in severity still come across, despite the supposed objectivity of the items. These difference can be overcome when automatically scoring specific text features. In the past decades, developments in computational linguistics, artificial intelligence, and psycholinguistics, have enabled the rise of techniques to analyze text features automatically. Several tools for automated essay scoring (AES) have been developed, and many validation studies have been reported. Instead of automatically providing an essay score, programs for text analyses could provide a score on different text features, thus contributing to a score for the aspect Correctness.

Furthermore, when considering the actual anchor items (i.e., the items where raters were prompted to place an essay on the scale) as individual items, an inter-rater agreement of .82 is achieved for each aspect. However, these figures cannot be interpreted reliably yet, because the raters were led to their final judgment by answering the analytical questions.

Further studies have to be conducted to gain insight into the individual strength of the anchor items. Still, these one-item assessments look promising and might well be developed into useful tools for classroom assessment because of their efficiency (cf. pair-wise comparison: Pollitt, 2004).

\section{Using a Combination of Methods to Assess Writing}

As shown in the studies mentioned in this chapter, different aspects of writing ability require different assessment methods. Since a writing product reveals very little about the cognitive processes taking place while producing the text, an objective test on certain components of the writing process (e.g., revision) can be a valuable addition. 
Such an indirect writing test can account for a reliable assessment of specific aspects of writing, shifting the focus from solely the product of the writing process to other relevant components and thus adding to the validity of a writing assessment. Furthermore, while the analytic evaluation of text structure benefits from the use of anchor essays, adopting merely the analytic questions is sufficient when assessing the content of a text. Automated text analyses, to conclude, can help in objectively scoring certain text features considered important to text quality, thus contributing to a score for correctness. Hence, assessing writing is not a matter of choice: a decent writing assessment should incorporate a mixture of assessment techniques - and benefit from it.

\section{References}

Breland, H., Camp, R., Jones, R. J., Morris, M. M., \& Rock, D. A. (1987). Assessing writing skill. New York, NY: College Entrance Examination Board.

Breland, H., \& Gaynor, J. L. (1979). A comparison of direct and indirect assessments of writing skill. Journal of Educational Measurement, 76, 119-128.

de Glopper, K. (1988). Schrijven beschreven. Inhoud, opbrengsten en achtergronden van het schrijfonderwijs in de eerste vier leerjaren van het voortgezet onderwijs [Writing described. Content, outputs and background of writing education in the first four years of secondary education] (Dissertation UvA). Den Haag, the Netherlands: SVO.

Feenstra, H. (2010a, June). Opstellen langs de meetlat. De constructie van een beoordelingsschaal voor schrijfproducten [Constructing a rating scale for writing products]. Poster presentation at the Onderwijs Research Dagen [Educational Research Days] 2010, Enschede.

Feenstra, H. (2010b, November). Assessing writing ability: Using anchor essays to enhance reliability. Paper presented at the 11th AEA-Europe Conference, Oslo, Norway.

Feenstra, H., \& Heesters, K. (2011a, May). Assessing writing through objectively scored tests: A study on validity. Paper presentation at the 8th EALTA Conference, Siena, Italy.

Feenstra, H., \& Heesters, K. (2011b, June). Objectieve schrijfvaardigheidstoetsen: een onderzoek naar validiteit [Objective writing tests: a study on validity]. Poster presented at the Onderwijs Research Dagen [Educational Research Days] 2011, Maastricht.

Flower, L., \& Hayes, J. R. (1981). A cognitive process theory of writing. College Composition and Communication, 32, 365-387. 
Godshalk, F. I., Swineford, F., \& Coffman, W. E. (1966). The measurement of writing ability. New York, NY: College Entrance Examination Board.

Knoch, U. (2011). Rating scales for diagnostic assessment of writing: What should they look like and where should the criteria come from? Assessing Writing, 16, 81-96.

Krom, R., van de Gein, J., van der Hoeven, J., van der Schoot, F., Verhelst, N., Veldhuijzen, N., \& Hemker, B. (2004). Balans van het schrijfonderwijs op de basisschool. Uitkomsten van de peilingen in 1999: halverwege en einde basisonderwijs en speciaal onderwijs [Report of the national assessment on writing education in primary schools. Outcomes of the surveys in 1999: Mid and end of primary education and education for special educational needs]. Arnhem, the Netherlands: Cito.

Lloyd-Jones, R. (1977). Primary trait scoring. In C. R. Cooper \& L. Odell (Eds.), Evaluating writing: Describing, measuring, judging (pp. 33-68). Urbana, IL: National Council of Teachers of English.

Messick, S. (1989). Validity. In R. Linn (Ed.), Educational measurement (3rd ed.). Washington, DC: American Council on Education.

Pollitt, A. (2004, June). Let's stop marking exams. Paper presented at the IAEA Conference, Philadelphia, PA.

Sijtstra, J. (Ed.). (1997). Balans van het taalonderwijs aan het einde van de basisschool 2. Uitkomsten van de tweede taalpeiling einde basisonderwijs [Report of the language education at the end of primary education 2. Outcomes of the second language survey end primary education]. Arnhem, the Netherlands: Cito.

Van den Bergh, H. (1990). Schrijfvaardigheid getoetst in het centraal schriftelijk eindexamen [Assessing writing ability within the central written examinations]. Levende Talen, $451,225-229$.

Van den Bergh, H., \& Rijlaarsdam, G. (1986). Problemen met opstelbeoordeling? Een recept [Issues with essay evaluation? A recipe]. Levende Talen, 413, 448-454.

Van Gelderen, A., Oostdam, R., \& van Schooten, E. (2010). Does foreign language writing benefit from increased lexical fluency? Evidence from a classroom experiment. Language Learning, 61, 281-321.

Weigle, S. C. (2002). Assessing writing. Cambridge, England: Cambridge University Press.

Wesdorp, H. (1974). Het meten van de produktief-schriftelijke taalvaardigheid. Directe en indirecte methoden: 'opstelbeoordeling' versus 'schrijfvaardigheidstoetsing' [Measuring productve-written language ability. Direct and indirect methods: 'essay rating' versus 'writing tests']. Purmerend, the Netherlands: Muusses. 
Zwarts, M. (Ed.). (1990). Balans van het taalonderwijs aan het einde van de basisschool. Uitkomsten van de eerste taalpeiling einde basisonderwijs [Report of the language education at the end of primary education 2]. Arnhem, the Netherlands: Cito. 


\title{
Chapter 8
}

\section{Don't Tie Yourself to an Onion: Don't Tie Yourself to Assumptions of Normality}

\author{
Maarten Marsman, Gunter Maris and Timo Bechger
}

\begin{abstract}
A structural measurement model (Adams, Wilson, \& Wu, 1997) consists of an item response theory model for responses conditional on ability and a structural model that describes the distribution of ability in the population. As a rule, ability is assumed to be normally distributed in the population. However, there are situations where there is reason to assume that the distribution of ability is nonnormal. In this paper, we show that nonnormal ability distributions are easily modeled in a Bayesian framework.
\end{abstract}

Keywords: Bayes estimates, finite mixture, item response theory, Gibbs sampler, Markov chain Monte Carlo, one-parameter logistic model, plausible values

\section{Introduction}

A structural measurement model (Adams, Wilson, \& Wu, 1997) consists of an item response theory (IRT) model for responses conditional on ability and a structural model that describes the distribution of ability in the population. At Cito, structural measurement models are used for test equating and to relate student characteristics to response behavior.

As a rule, we assume that ability is normally distributed in the population. We do this because it is easy and because we often do not have a clear alternative. However, there are situations where we have reason to assume that ability is not normally distributed in the population, for instance, when we know that the population consists of students who are selected from a larger, possibly normal population, based on one of our own tests. Thus, the onion in our title refers to the assumption of normality to which structural measurement models seem intimately tied. The most common form of nonnormality of the ability distribution found in Cito applications is skewness. This has enticed Molenaar (2007) and Verhelst (2008) to develop more general models that can handle skew ability distributions. They developed complex procedures to estimate these models using maximum likelihood methods, and the procedure developed by Verhelst is implemented in the Cito program SAUL. 
We propose to use a (finite) mixture of normal distributions to model different forms of nonnormality, such as skewness, kurtosis, and multimodality. The structural measurement model is formulated in a Bayesian framework, and the Gibbs sampler is used to estimate the parameters. The Gibbs sampler requires a sample from the posterior distribution of ability, and the mixture plays the role of the prior distribution of ability. Draws from the posterior of ability are called plausible values (PVs; Marsman, Maris, Bechger, \& Glas, 2011; Mislevy, 1991), and once they are obtained, estimating the parameters from the mixture becomes a routine exercise.

The structure of the paper is as follows. First, we introduce a real-data example to motivate our concerns about the assumption of normality. Then, we outline a Bayesian procedure that is then applied to the data of the motivating example. The paper ends with a discussion.

\section{Motivating Example: Entreetoets Data}

We use data of $N=136,495$ students responding to $k=39$ math items of the Cito Entreetoets. The measurement model is the one-parameter logistic model (OPLM; Verhelst \& Glas, 1995). Since the OPLM is an exponential family (EF) IRT model, it can be fitted independently from the structural model using conditional likelihood methods. The parameters were estimated and showed reasonable fit.

We estimated the parameters of the structural model using the Gibbs sampler as described in Marsman et al. (2011) using noninformative priors. Marsman et al. (2011) developed several algorithms that use the method of composition (Tanner, 1993) to sample PVs from the posterior distribution of ability conditional on the response data. Because the OPLM is in the EF, the posterior of ability is characterized by its sufficient statistic, and the conditional composition algorithm for EF IRT models (the CC-EF algorithm) can be used. Furthermore, Marsman et al. show how recycling intermediate candidate values can increase efficiency when students come from few marginal distributions using the CC-EF-R algorithm.

The Markov chain Monte Carlo (MCMC) algorithm ran for 1,000 iterations, which is sufficient due to the low amount of autocorrelation and thus results in almost immediate convergence of the Markov chain. The expected a posteriori (EAP) estimates and posterior standard deviations are $\hat{\mu}=0.1804185$ (0.0004750) and $\hat{\sigma}=0.1599017$ (0.0003887).

To study the fit of the estimated model, we compared the observed (weighted sum) score distribution with the generated score distribution under the model; see Figure 1. 


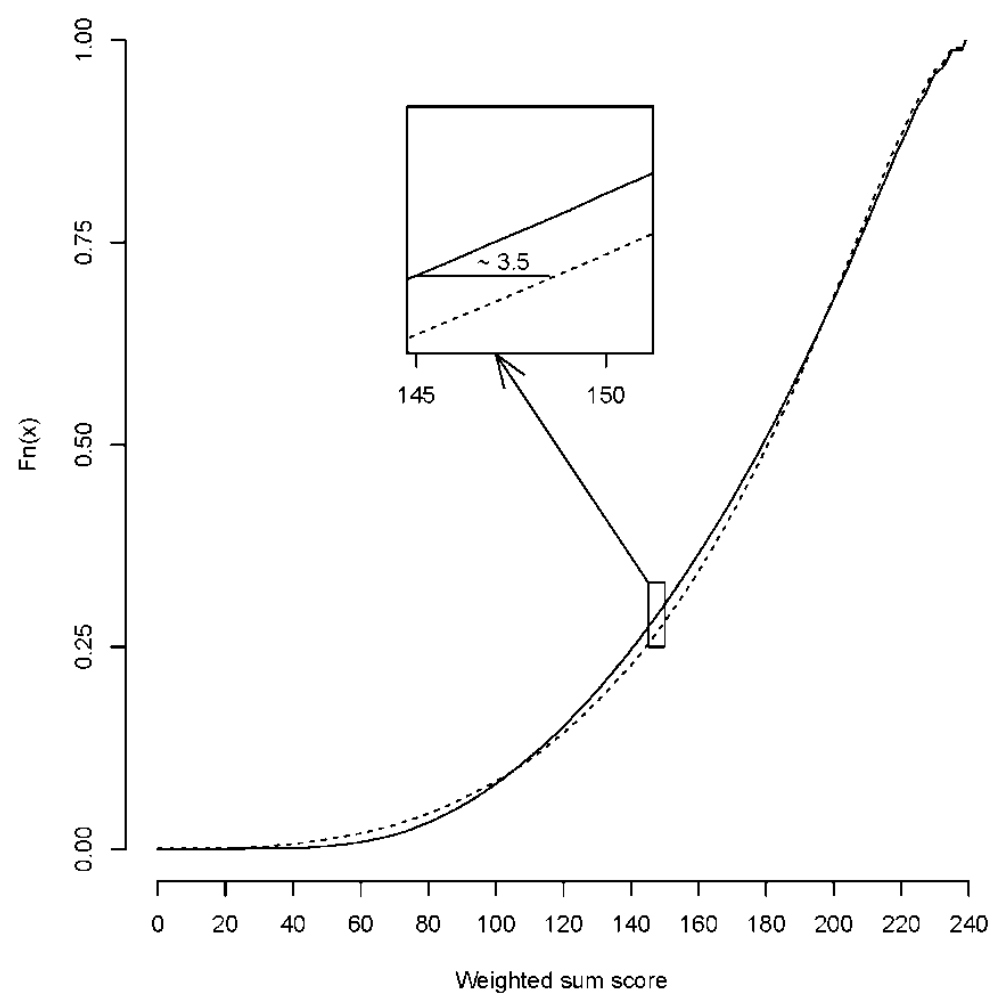

Figure 1 Observed (solid) and replicated (dashed) weighted score distributions

There were discrepancies between the observed and generated data. The magnified section in Figure 1 shows a section of the score distributions where they differ in approximately 3.5 score points. The geometric mean of the item weights in the OPLM model was set at 6 , so a difference of 3.5 points would be a little less than 0.6 raw score points. 
Table 1 Equating the Score Distributions

\begin{tabular}{llllll}
\hline Score & Observed & $\begin{array}{l}\text { Generated } \\
\text { with normal }\end{array}$ & $\begin{array}{l}\text { Generated } \\
\text { with mixture }\end{array}$ & $\begin{array}{l}\text { Difference } \\
\text { with normal }\end{array}$ & $\begin{array}{l}\text { Difference } \\
\text { with mixture }\end{array}$ \\
\hline 0 & 0 & 8 & 0 & 8 & 0 \\
25 & 11 & 282 & 45 & 271 & 34 \\
50 & 455 & 1,602 & 700 & 1,147 & 245 \\
75 & 3,309 & 4,981 & 3,514 & 1,672 & 205 \\
100 & 11,000 & 11,358 & 10,730 & 358 & 270 \\
125 & 23,671 & 22,080 & 23,672 & 1,591 & 1 \\
150 & 41,325 & 38,513 & 41,757 & 2,812 & 432 \\
175 & 64,197 & 62,108 & 64,184 & 2,089 & 13 \\
200 & 92,872 & 93,487 & 92,882 & 615 & 10 \\
225 & 125,543 & 126,655 & 125,473 & 1,112 & 70 \\
\hline
\end{tabular}

The observed differences may have large implications in test equating. This is illustrated in Table 1, which contains six columns. The first column is a score used as a possible cut-off point in an equating procedure. The number of persons who received that score or lower as observed in the sample or generated with a normal density are given in the second and third columns, respectively. In the fifth column, we look at the difference between the number of persons we observe with what we expect under the model. These differences are not anywhere near zero, illustrating that model misfit can have large implications.

\section{Using Plausible Values}

To estimate the parameters from the structural model, we used PVs, which are draws from the posterior of ability. We want the PVs to have the same distribution as ability in the population. 
However, since we do not know how ability is distributed, we introduce a structural model and use it as a prior distribution. The posterior distribution now has two ingredients:

1. The likelihood (IRT model): we can add more items, which will make the likelihood dominate the prior distribution so that the posterior converges to the true posterior distribution.

2. The prior distribution: we can adjust the prior to match information in the likelihood, so that the likelihood more easily dominates the prior and the posterior converges to the true posterior distribution.

When the measurement model is firmly established, it is guaranteed that the distribution of PVs is closer to the true posterior distribution than the estimated structural model. We illustrate this in Figure 2(a), where a histogram of generated PVs with a normal distribution are given along with the estimated normal density. Clearly, the measurement model makes the PV distribution negatively skewed and as a result does not match the prior distribution.

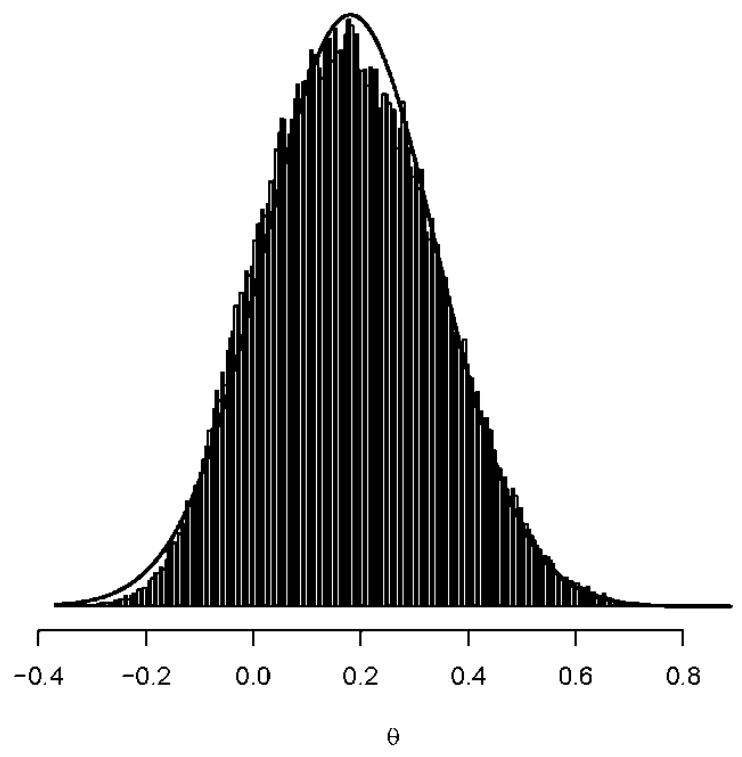

(a) Normal population model.

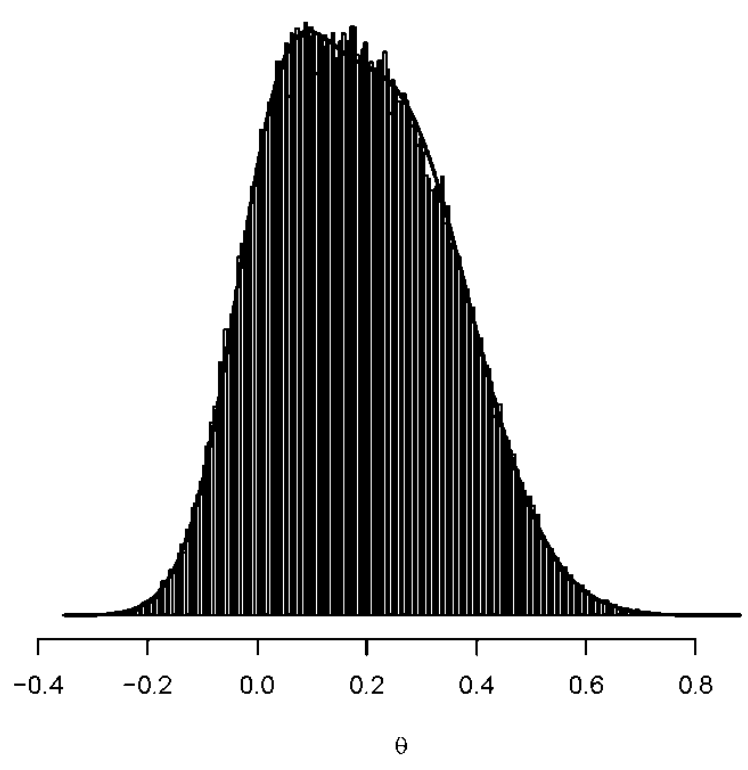

(b) Mixture population model.

Figure 2 Histograms of PVs and estimated density 


\section{Motivating Example: Reanalysis}

We can speed up convergence of the PV distribution to the true posterior distribution by improving the fit of the structural model and adjusting it to better match the information in the likelihood. We do this by using a mixture of two normal distributions. Standard methods for estimating a normal mixture using Gibbs samplers are readily available, and we refer the interested reader to Congdon (2010, Chapter 3) or Fox (2010, Chapter 6).

The assignment to a component was modeled as a binomial random variable and given a flat Beta prior, uniform over the range $[0,1]$. The parameters from the structural model were assigned noninformative priors as in the previous example. This is not convenient when there is a risk of (almost) empty mixture components, in which case informative priors can be assigned. The parameters from the mixture distribution were estimated using the Gibbs sampler, which ran for 5,000 iterations. Convergence of the estimated distribution was relatively fast as can be seen by inspecting the traceplots of the mean, variance, skewness, and kurtosis of the mixture model, shown in Figure 3. 


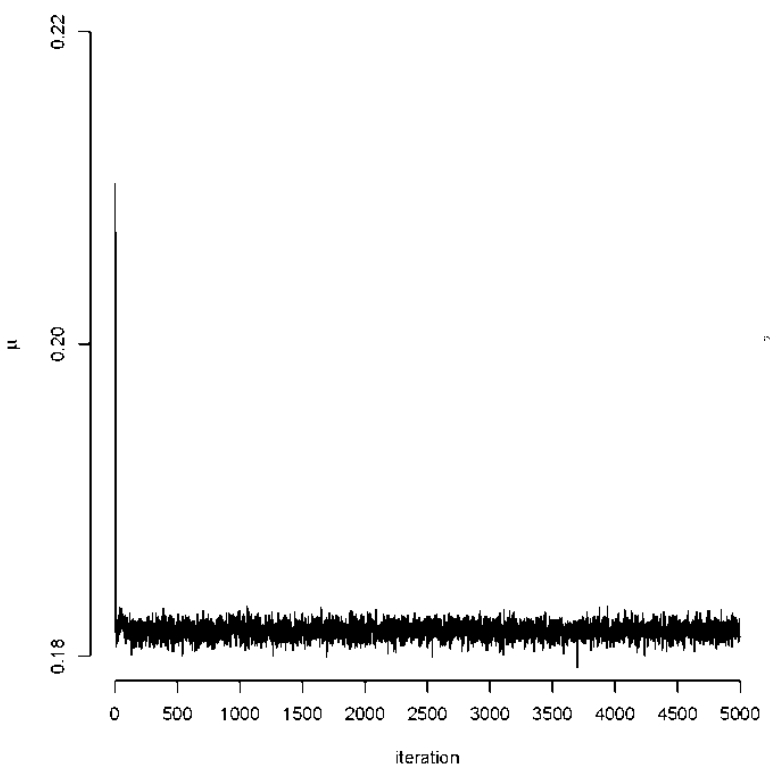

(a) Mean of mixture model.

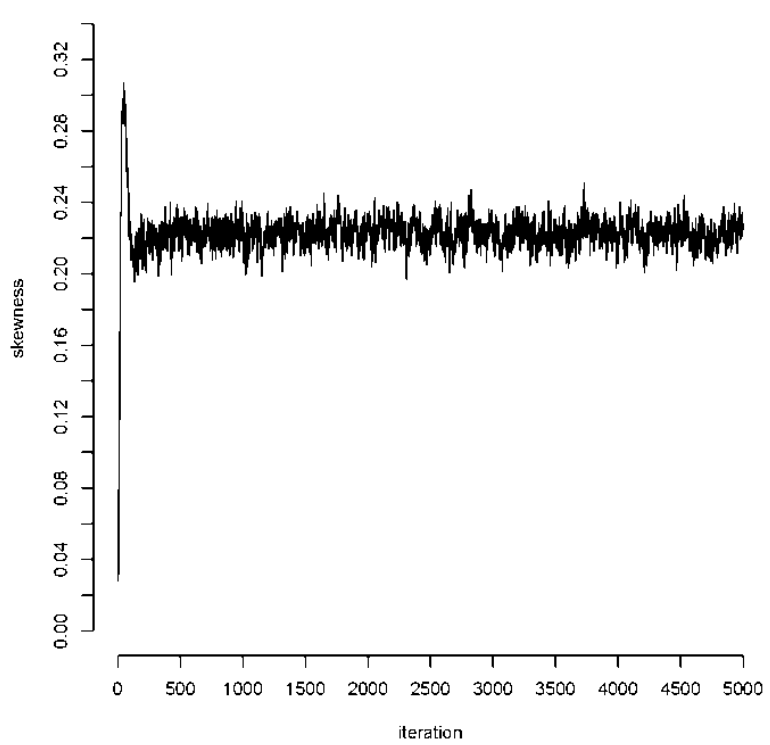

(c) Skewness of mixture model.

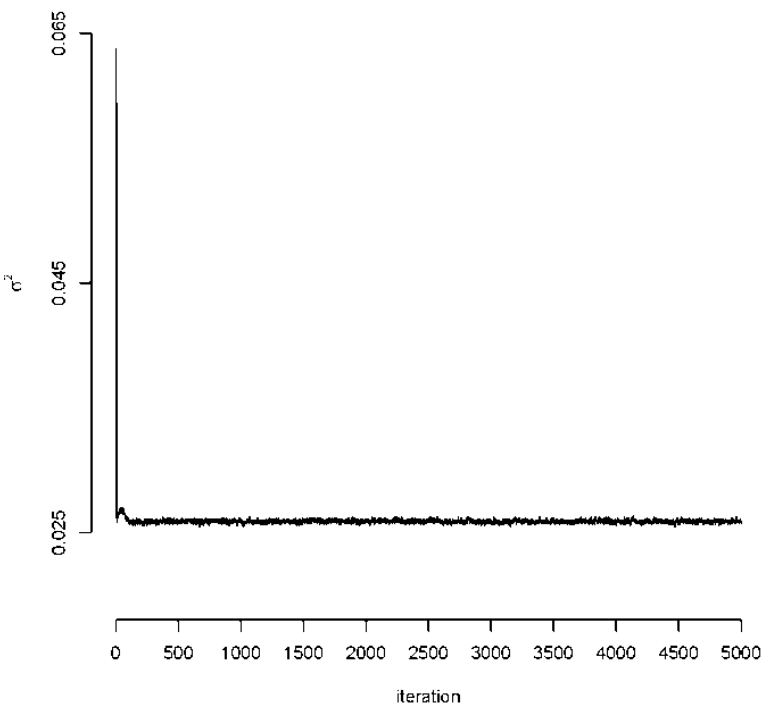

(b) Variance of mixture model.

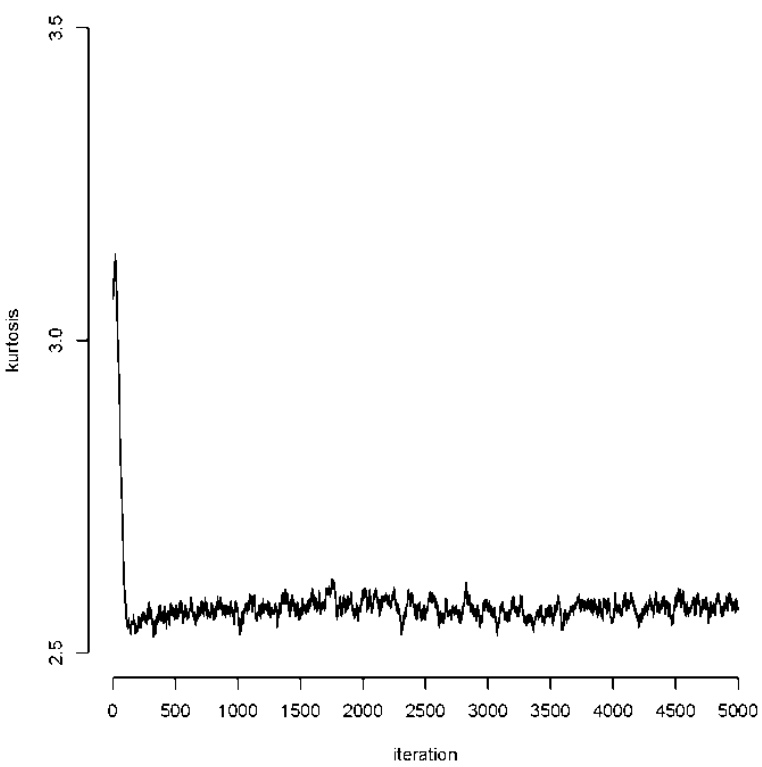

(d) Kurtosis of mixture model.

Figure 3 Trace plots of the mean, variance, skewness, and kurtosis of the mixture distribution 
Because of more freely estimating the population model, the distribution of PVs converged to the true posterior ability distribution. This can be seen in Figure 2(b), where a histogram of generated PVs with the mixture distribution are given along with the estimated density. The distribution of PVs and the estimated structural model are aligned, confirming that it has converged to the true posterior distribution. Since PVs are now a sample from the structural model, we can use them for other purposes. For instance, in large-scale educational surveys, such as the Program for International Student Assessment (PISA) and the European Survey on Language Competences (ESCL), PVs are provided for secondary analyses.

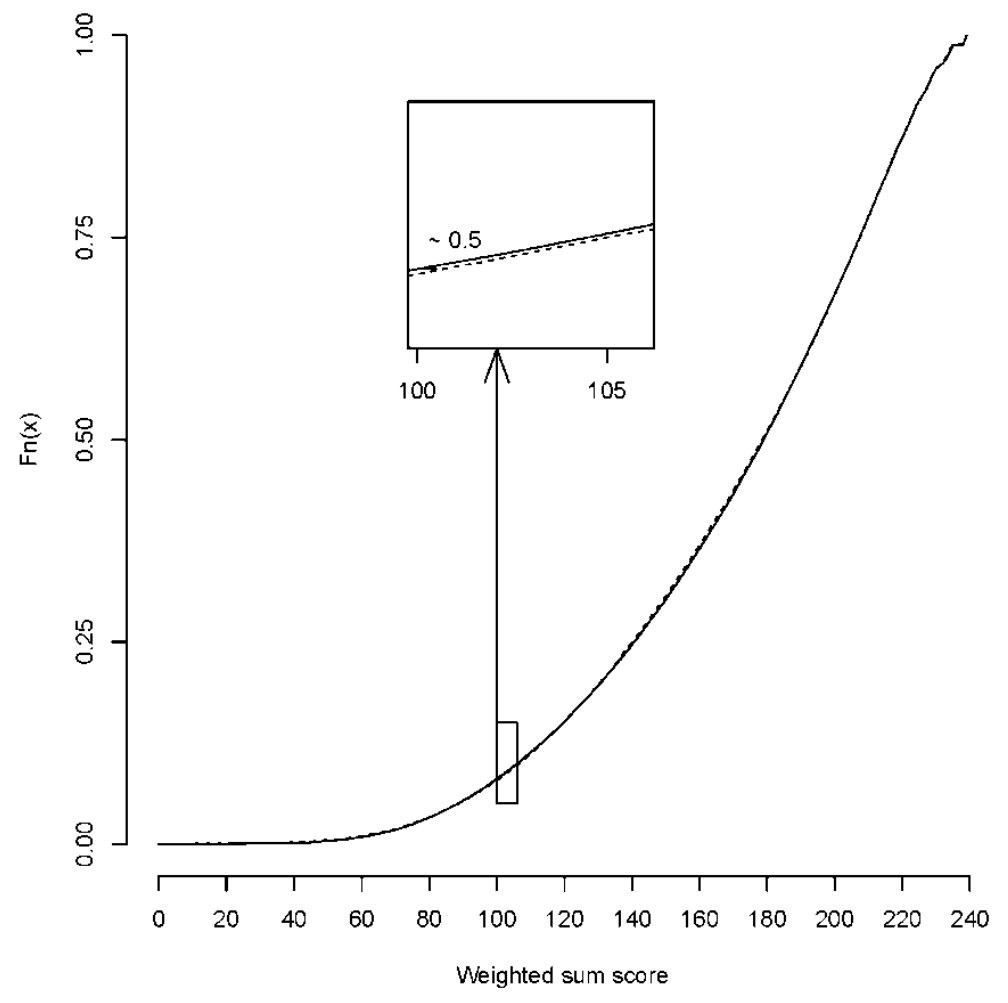

Figure 4 Observed (solid) and replicated (dashed) weighted score distributions at iteration 2,000

The fit of the structural measurement model was assessed via a comparison of observed and replicated score distributions as before; see Figure 4. The mixture distribution provides a better fit to the data than the normal distribution as provided.

The magnified section in Figure 4 shows that there are still discrepancies, although they are less severe than those found in Figure 1. The observed difference of less than of 0.5 points on the weighted score scale refers to less than 0.1 raw score points, approximately. 
This can still have a large effect in test equating. The fourth column in Table 1 shows the number of persons who received the cut-off score or lower as generated with a mixture. The sixth column shows the difference between the number of persons we observed with what we expected under the mixture model. Clearly these numbers are still substantial. The question is: do we have to explain the results to the parents of 2,812 or of 432 children, which is large either way, but substantially larger under the normality assumption.

\section{Discussion}

As a rule, assumptions of normality (the onion) are introduced in test equating, although situations exist where there is reason to believe that the ability distribution is not normal. In this paper, we showed that we can easily model deviations from normality in a Bayesian framework by using PVs and a mixture distribution as a prior. Compared to regular applications of mixture distributions, we are not interested in its components but use it merely for curve-fitting. As an illustration, we applied a mixture of two normal distributions to Entreetoets data. It is easy to extend the mixture to include more components at a low cost in terms of additional parameters. The mixture is flexible and can model many deviations from normality, such as skewness, kurtosis, and multimodality.

In addition, ignoring deviations from normality can have serious effects on test equating. Furthermore, if the ability distribution shows similar deviations in repeated assessments and these deviations are ignored, the effects can add up in the equating procedure. As a result, the projected norm can drift away from the originally proposed norm. Thus, it is very important to correctly model the ability distribution to provide valid inference in test equating. The mixture solution is easily applied in test equating, and the Entreetoets example shows that it can improve model fit and consequently provide better predictions. 


\section{References}

Adams, R., Wilson, M., \& Wu, M. (1997). Multilevel item response models: An approach to errors in variables regression. Journal of Educational and Behavioral Statistics,22,47-76.

Congdon, P. (2010). Applied Bayesian hierarchical methods. Boca Raton, FL: Chapman \& Hall/CRC Press.

Fox, J. (2010). Bayesian item response modeling. New York, NY: Springer.

Marsman, M., Maris, G., Bechger, T., \& Glas, C. (2011). A conditional composition algorithm for latent regression. (Measurement and Research Department Report No. 11-2). Cito.

Mislevy, R. (1991). Randomization-based inference about latent variables from complex samples. Psychometrika, 56, 177-196.

Molenaar, D. (2007). Accounting for non-normality in latent regression models using a cumulative normal selection function. (Measurement and Research Department Report No. 07-3). Cito.

Tanner, M. (1996). Tools for statistical inference (3rd ed.). New York, NY: Springer.

Verhelst, N. (2008). Untitled document containing updated theory and a short addition to the manual for the structural analysis of a univariate latent variable (SAUL) program. Cito.

Verhelst, N., \& Glas, C. (1995). Rasch models; foundations, recent developments, and applications. In G. Fischer \& I. Molenaar (Eds.), One Parameter logistic model (pp. 215238). New York, NY: Springer-Verlag. 


\title{
Chapter 9
}

\section{Towards a Comprehensive Evaluation System for the Quality of Tests and Assessments}

\author{
Saskia Wools
}

\begin{abstract}
To evaluate the quality of educational assessments, several evaluation systems are available. These systems are, however, focused around the evaluation of a single type of test. Furthermore, within these systems, quality is defined as a non-flexible construct, whereas in this paper it is argued that the evaluation of test quality should depend on the test's purpose. Within this paper, we compare several available evaluation systems. From this comparison, design principles are derived to guide the development of a new, comprehensive quality evaluation system. The paper concludes with an outline of the new evaluation system, which intends to incorporate an argument-based approach to quality.
\end{abstract}

Keywords: Standards, evaluation, quality, educational assessment, argument-based approach

\section{Introduction}

In all levels of education, students have to take tests and assessments to demonstrate their ability, for example, to show whether they have fulfilled the course objectives or to guide them in their further learning. In the context of high-stakes exams and assessments, the importance of good quality decisions is clear. However, in other contexts, the assessment results need to be valid and reliable too. In other words, despite the stakes of an exam, the results need to be appropriate for its intended use. This can only occur when the assessment instruments that are used to assess the students are of good quality. To evaluate test quality, several evaluation systems and standards are available. The currently available evaluation systems, however, tend to focus around one specific type of test or test use, for example, computer-based tests (Keuning, 2004), competencebased assessments (Wools, Sanders, \& Roelofs, 2007), examinations (Sanders, 2011), or psychological tests (Evers, Lucassen, Meijer, \& Sijtsma, 2010). Standards are often more broadly defined, but are aimed at guiding test developers during the development process and are not suited for an external evaluation of quality. 
The purpose of this paper is to introduce the outline of a new evaluation system that will be more flexible and comprehensive than the currently available evaluation systems. Furthermore, this proposed evaluation system is not only suitable to guide test development, but can also be used as an instrument for internal or external audits. In the first section of this paper, the available standards and evaluation systems are described. In the second section, the principles that serve as a basis for the new evaluation system are specified. From this second section, we will derive the design of the new system that is described in the final section of this paper.

\section{Section 1 - Guidelines, Standards and Evaluation Systems}

To describe the currently available systems for the evaluation of test quality, we will compare nine quality evaluation systems. The nine systems will be compared based on their purpose, their intended audience, and their object of evaluation. We do not aim to include all of the available evaluation systems, nor will we describe every aspect for every system that is mentioned, since this section is meant mainly to exemplify the diversity of the systems.

We will differentiate between guidelines, standards, and evaluation systems. Guidelines suggest quality aspects that you can comply with. Standards mention aspects of quality that you should comply with, in order to develop sound and reliable tests. Evaluation systems focus on evaluating a test, and prescribe what quality aspect must be met to ensure minimal quality. We will also add criteria to the comparison that are mentioned by researchers as being important, but that are not implemented in the guidelines, standards, or evaluation systems.

\section{Systems for Comparison}

Guidelines:

1. International guidelines for test use from International Testing Committee (ITC) (Bartram, 2001)

Standards:

2. Standards for educational and psychological testing (AERA, APA, \& NCME, 1999)

3. European framework of standards for educational assessment (AEA-Europe, 2012) 
4. ETS standards (Educational Testing Service (ETS), 2002)

5. Cambridge approach (Cambridge Assessment, 2009)

6. Code of fair testing practices in education (Joint Committee on Testing Practices (JCTP), 2004).

Evaluation systems:

7. COTAN evaluation system for test quality (Evers et al., 2010)

8. EFPA review model for the description and evaluation of psychological tests (Lindley, Bartram, \& Kennedy, 2004)

\section{Criteria:}

9. Quality criteria for competence assessment programs (Baartman, Bastiaens, Kirschner, \& van der Vleuten, 2006) 
Table 1 Comparison of standards, guidelines, and evaluation systems

\begin{tabular}{|c|c|c|c|c|c|c|c|c|c|}
\hline & ITC & $\begin{array}{l}\text { AERA } \\
\text { Standards }\end{array}$ & $\begin{array}{l}\text { AEA- } \\
\text { Europe }\end{array}$ & ETS & $\begin{array}{l}\text { Cambridge } \\
\text { Assessment }\end{array}$ & JCTP & COTAN & EFPA & Baartman \\
\hline \multicolumn{10}{|l|}{ Purpose } \\
\hline $\begin{array}{l}\text { Guide test } \\
\text { development }\end{array}$ & & & $\mathrm{X}$ & $\mathrm{X}$ & $\mathrm{X}$ & $\mathrm{X}$ & & & \\
\hline Guide test use & $\mathrm{X}$ & $\mathrm{X}$ & $\mathrm{X}$ & & & $\mathrm{X}$ & & & \\
\hline Guide self-evaluation & & & $\mathrm{X}$ & & & & & & $\mathrm{X}$ \\
\hline Guide audits & & $\mathrm{X}$ & $\mathrm{X}$ & & & & $\mathrm{X}$ & $\mathrm{X}$ & \\
\hline \multicolumn{10}{|l|}{ Intended audience } \\
\hline Test specialists & & $\mathrm{X}$ & $\mathrm{X}$ & & & & $\mathrm{X}$ & $\mathrm{X}$ & \\
\hline Teachers & $\mathrm{X}$ & & $\mathrm{X}$ & & & $\mathrm{X}$ & & & $\mathrm{X}$ \\
\hline Users & & $\mathrm{X}$ & $\mathrm{X}$ & & & $\mathrm{X}$ & $\mathrm{X}$ & & $\mathrm{X}$ \\
\hline Companies & & & $\mathrm{X}$ & $\mathrm{X}$ & $\mathrm{X}$ & & & & \\
\hline
\end{tabular}

\section{Object of evaluation}

Construction process

Educational

assessment

Competence

assessment

$\begin{array}{lll}\mathrm{X} & \mathrm{X} & \mathrm{X}\end{array}$

Psychological tests

$\mathrm{X}$

Test product and use

Educational

assessment

Competence

assessment

$\begin{array}{lll}\mathrm{X} & \mathrm{X} & \mathrm{X}\end{array}$

$(\mathrm{x})^{*} \quad \mathrm{x}$

Psychological tests

$\mathrm{x}$

(x)

Note *Although COTAN's focus lies on psychological tests, the system is also used to evaluate educational assessments

Table 1 displays all of the systems for comparison and the three aspects that they are compared on. The object of evaluation is divided into two main objects: construction process and test product and use. Systems aimed at evaluating the process tend to give guidelines for developing solid tests, whereas systems that focus on the test product and use are meant for auditing a fully developed test that is already in use. One element that stands out from this table is that the AEA-Europe system is multi-functional.

That system aims to be a framework of standards that can be used in several different ways and for all sorts of educational assessments. In the remainder of this section, we will compare the systems in detail for each of the three aspects in Table 1. 


\section{Purpose}

In our comparison, we distinguished four main purposes for the quality evaluation systems, guidelines, and standards. First, we looked at systems aiming to guide test development. These systems try to help the test developer in constructing a sound test. Both the ETS standards and the Cambridge approach are meant to guide test development. Another purpose is to help users apply tests properly and to make them aware of the risks when they do not follow protocol. One example of a system that has the purpose of helping users understand the interpretation of test scores is the ITC document that has guidelines for test use. Some systems are meant for selfevaluation by the test constructors, to help them identify the strong and weak points of their assessment; Baartman formulated criteria for this specific purpose. Finally, we included systems meant for audit purposes. In this case, an external expert audits the quality of the test by means of an evaluation system, such as the COTAN system or the EFPA system.

\section{Intended Audience}

The intended audience of the evaluation systems can be test specialists, teachers, users, or companies. However, most of the systems that we compare are developed for multiple audiences. The systems that have only one intended audience are the ITC document (teachers), the Cambridge approach and ETS standards (companies), and EFPA (test specialists). The COTAN and AERA systems are meant for test specialists as well as teachers. The JCTP standards are intended for both teachers and test users.

\section{The Object of Evaluation}

The definition of quality also varies across the different systems. Some systems focus on the construction process, while others focus on the fully developed test and its use. For example, COTAN focuses on the fully developed test product and not on the development process. ITC, however, intends to evaluate the development process. At the same time, the type of test differs: JCTP focuses on classroom assessment, while Baartman focuses on competence assessment programs. The AEA-Europe framework of standards focuses on educational assessment in general, where COTAN aims at both psychological and educational tests. 


\section{Issues With the Currently Available Systems}

One problem with all of these evaluation systems is that quality is defined as a non-flexible construct. These systems provide criteria that should be met, while it is actually more appropriate to choose criteria that fit the intended use of the test. Doing this would also provide the possibility of weighing the criteria according to the purpose of the test. This might solve the problem of having to create a new evaluation system for every type of test. Once the purpose of the test defines the selected criteria, we can also evaluate several types of tests with the system.

Another problem with these evaluation systems is the process of evaluating the tests. To evaluate a test as part of an external audit, one needs to look through all of the testing materials and supporting documents that include the results of the trial administrations of the test, validation studies, and other evidence that is considered relevant for the audit. However, it depends on the auditor whether all of the evidence is found. Moreover, going through all of these documents is not a very time efficient way to evaluate tests, and a lot of both content and methodology expertise is needed to evaluate a complete assessment (Wools, Sanders, Eggen, Baartman, \& Roelofs, 2011). When a new evaluation system can make classifying evidence a task for test developers, the auditors only have to look through the relevant evidence. And when all of the evidence is structured in advance, it is also possible to give a part of the test to an auditor who knows the content and another part to an auditor who specializes in methodology.

These issues are addressed as principles in the outline of the proposed evaluation system. The design of the new system tries to gain from existing evaluation systems as well. In the remainder of this paper, the design of the new system is described.

\section{Section 2 - Principles of the New Evaluation System}

In the new evaluation system, quality is defined as the degree to which something is useful for its intended purpose. In testing and assessment practice, the variety of intended purposes is very large and, furthermore, the solutions chosen to reach those purposes are endless. And, when quality is defined as being dependent on the purpose of a test, it seems hard, or even impossible, to develop an evaluation system with fixed criteria that are suitable for all possible tests and assessments. Therefore, we do not aim to develop the right set of criteria that can be used to evaluate all possible tests. 
The main idea behind this system is for it to be used to build an argument that helps test developers to show that a test or assessment is sufficiently useful for its intended purpose. To build this argument, evidence is needed to convince the public of the test's usability. This evidence is established, collected, and presented during the test's development process.

The argument-based approach to quality is derived from the argument-based approach to validation, as described by Kane $(2004 ; 2006)$. The remainder of this section extracts the argument-based approach to quality into the underlying principles of the new evaluation system. As a starting point for the specification of the principles, the purpose of the system is addressed.

\section{Purpose}

The purpose of the system is to evaluate the quality of tests and assessments on several occasions during the construction of a test. It might be used during the development stage to indicate weak spots that need attention or adaptation, or utilized to point out aspects that are in need of evidence in order to enhance the plausibility of the argument that is being built. When the development stage is finished, the system also needs to facilitate an external evaluation of the test. The criteria used are derived from existing evaluation systems, and may be chosen or combined based on the purpose of the test or the purpose of the evaluation.

\section{Content}

As mentioned before, quality is defined as the degree to which something is useful for its purpose. By taking an argument-based approach to quality, it is possible to interpret quality as an integral entity instead of a combination of isolated elements. This entails the possibility of an assessment to compensate for weaker points with strong points. Furthermore, this view does justice to the fact that all aspects of an assessment are linked and cannot be evaluated without considering the others.

This view also implies that the instrument that is used to assess students and to generate scores cannot be evaluated without considering the use of these scores. In an argument-based approach to quality, the use of the scores, or the decision that is made based upon the scores, guides the test developer in determining the appropriate quality standards. This means that, on one hand, the intended decision resulting from a test is the main determiner in choosing the criteria that are necessary to evaluate the appropriateness of the test. 
On the other hand, the degree to which the test must comply with the standards is also based upon the intended decision. For a high-stakes certification exam that consists of 40 multiple-choice items, reliability, IRT model-fit, and validation by means of an external criterion might be more appropriate than any coefficient of inter-rater reliability. Whereas, in a selection procedure where two assessors are interviewing their own groups of students, inter-rater reliability and comparability seem to be the most important aspects.

\section{Process}

According to the argument-based approach to quality, an argument is built and evidence is collected, selected, and presented according to the shape of the argument. By selecting and presenting the appropriate evidence, the evaluation is prepared during the test construction phase. Once the (external) audit starts, the auditor does not need to go through all of the available material, but only investigates the evidence that is presented according to the structure of the argument. This not only makes the evaluation process more manageable for the auditor, but also enhances the comparability of the ratings of different auditors, because they all took the same evidence into account. Another advantage of structuring the evidence before auditing is that different auditors with different competencies, for example, psychometricians and content experts, can evaluate the parts that they specialize in.

\section{Relationship to Other Evaluation Systems}

One of the reasons to evaluate test quality is that it is necessary to decide whether the use of a certain test for an intended decision is justified. We would like to know whether a test is good enough for the stated purpose. An argument that is built and accompanied with evidence and that is evaluated as plausible is, unfortunately, not an answer to the question of whether a test is good enough.

Therefore, the new evaluation system also includes other evaluation systems' criteria that do lead to a result that states whether a test is good enough. These criteria are built into the system in such a way that, once the evidence is structured in the different elements of the argument, the criteria will appear in clusters that match the order of the argument. 
The order of the criteria is different from the order in the original evaluation systems, but once every criterion is answered, the results will be presented according to the elements of the original evaluation systems. For example, COTAN's criteria are clustered differently, but the evaluation results will be presented in the seven categories that are distinguished by COTAN.

\section{Section 3 - Design of the New Evaluation System}

The new evaluation system will be a computer application that consists of several modules. These modules are: design, evidence, evaluation, and report. The application is designed for use during the test development process, but can also be used for the evaluation of existing tests. However, once the existing tests are evaluated, the test constructors have to prepare the evaluation by designing and structuring the argument.

\section{Design Module}

This module delivers the outline of an argument. Therefore, several steps need to be taken. To make sure a user will complete all of the necessary fields, this module is wizard based. It starts by posing questions about the characteristics of the test. Once the general information about the test is collected, the assumptions and inferences that underlie the quality argument are specified. To build the argument, first the focus will be on the shape of the argument. The amount of inferences that need to be specified depends, for example, on the purpose of the test. When the shape of the argument and the characteristics of the test are known, the actual building of the argument starts. For every inference, the underlying assumptions are described. Furthermore, possible counter arguments are also made explicit.

\section{Evidence Module}

The evidence module consists of two parts. First, it facilitates the storage and structuring of the sources of evidence. In this module, a user can upload documents, graphical representations, research reports, or test materials.

For every document that is uploaded, it is possible to enter a short description and to add tags. These tags can be used in the evaluation module to help auditors select the right sources of evidence. Second, it focuses on structuring and classifying evidence. Evidence can be selected and added to the inferences that are specified in the design module. 
Graphics show which inferences are backed up with evidence so that the user can see which inferences need more evidence.

\section{Evaluation Module}

This module is designed to facilitate the evaluation process by combining the information given in the design and evidence module. Therefore, there are two main parts within this module: prepare and evaluate.

Within the prepare section, the test developer can choose the evaluation system that will judge the test and the argument can be reviewed. The evaluate section shows the specified argument and the uploaded evidence. Furthermore, the criteria, questions, or aspects from the chosen evaluation system are shown with every inference. An auditor can go through the inferences and evaluate the quality of the evidence based upon the given criteria. Only the evidence that is a part of an inference is shown, therefore, the auditor does not need to look for the appropriate evidence.

\section{Report Module}

The report module can be used to retrieve the results of the evaluation. It can also be used to print parts of the argument or the accompanying evidence, for example, to construct a test manual that incorporates all of the evidence and that is structured according to the specified argument.

\section{Conclusion}

This paper outlines a new evaluation system for the quality of tests, assessments, and exams. The new evaluation system will be developed as part of a study that will be shaped according to the principles of design research (Plomp, 2007) and will be finished in the summer of 2013. This system will incorporate an argument-based approach to quality, and we will suggest a computer application that can be used to gather, structure, and evaluate the evidence of quality.

By explicitly using sources of evidence that are created during the different phases of the test development process, this new evaluation system will bring new awareness of quality issues to everyone involved in test development. 
The argument-based approach to quality is based upon a theory used in validation practice. This gives us the opportunity to look at quality in a more comprehensive way. From here, it is also possible to evaluate and weigh evidence in respect to the purpose of the test. Furthermore, where other evaluation systems focus on the end product of the test development phase (the test), this new evaluation system bridges the development efforts to the end product.

This new evaluation system will, however, also include existing quality criteria, which makes an evaluation according to the existing evaluation systems still possible. In conclusion, the proposed evaluation system will allow us to evaluate test quality in a flexible and comprehensive way, and gives us a conclusion about test quality from other evaluation systems at the same time. Could this be the system that combines the best of both worlds?

\section{References}

AEA-Europe. (2012). European framework of standards for educational assessment. Retrieved from http://www.aea-europe.net/index.php/professional-development/standards-for$\underline{\text { educational-assessment }}$

AERA, APA, \& NCME. (1999). Standards for educational and psychological testing. Washington, DC: American Educational Research Association.

Baartman, L., Bastiaens, T., Kirschner, P., \& van der Vleuten, C. (2006). The wheel of competency assessment: Presenting quality criteria for competency assessment programs. Studies in Educational Evaluation, 32(2), 153-170.

Bartram, D. (2001). The development of international guidelines on test use: The international test commission project. International Journal of Testing, 1(1), 33-53.

Cambridge Assessment. (2009). The Cambridge approach. Principles for designing, administering and evaluating assessment. Cambridge: Cambridge Assessment.

Educational Testing Service (ETS). (2002). ETS standards for quality and fairness. Princeton, NJ

Evers, A., Lucassen, W., Meijer, R., \& Sijtsma, K. (2010). COTAN beoordelingssysteem voor de kwaliteit van tests. Amsterdam: NIP.

Joint Committee on Testing Practices. (2004). Code of fair testing practices in education. Washington, DC: Joint Committee on Testing Practices.

Kane, M. T. (2004). Certification testing as an illustration of argument-based validation. Measurement, 2, 135-170. 
Kane, M. T. (2006). Validation. In R. Brennan (Ed.), Educational measurement (4th ed.) (pp. 1764). Westport, CT: American Council on Education and Praeger Publishers.

Keuning, J. (2004). De ontwikkeling van een beoordelingssysteem voor het beoordelen van “Computer Based Tests.” POK Memorandum 2004-1. Arnhem: Citogroep.

Lindley, P., Bartram, D., \& Kennedy, N. (2004). EFPA review model for the description and evaluation of psychological tests. Retrieved from http://www.efpa.eu/professionaldevelopment/tests-and-testing

Plomp, T. (2007). Educational design research: An introduction. In T. Plomp \& N. Nieveen (Eds.), An introduction to educational design research (pp. 9-35). Enschede, Nederland: SLO.

Sanders, P. (2011). Beoordelingsinstrument voor de kwaliteit van examens. Enschede: RCEC.

Wools, S., Eggen, T., \& Sanders, P. (2010). Evaluation of validity and validation by means of the argument-based approach. CADMO, 8, 63-82.

Wools, S., Sanders, P., Eggen, T., Baartman, L., \& Roelofs, E. (2011). Evaluatie van een beoordelingssysteem voor de kwaliteit van competentie-assessments. Pedagogische Studiën, 88, 23-40.

Wools, S., Sanders, P., \& Roelofs, E. (2007). Beoordelingsinstrument: Kwaliteit van competentie assessment. Arnhem: Cito. 


\title{
Chapter 10
}

\section{Influences on Classification Accuracy of Exam Sets: An Example from Vocational Education and Training}

\author{
Marianne Hubregtse and Theo J.H.M. Eggen
}

\begin{abstract}
Classification accuracy of single exams is well studied in the educational measurement literature. However, when making important decisions, such as certification decisions, one usually uses several exams: an exam set. This chapter elaborates on classification accuracy of exam sets. This is influenced by the shape of the ability distribution, the height of the standards, and the possibility for compensation. This is studied using an example from vocational education and training (VET). The classification accuracy for an exam set is computed using item response theory (IRT) simulation. Classification accuracy is high when all exams from an exam set have equal and standardized ability distributions. Furthermore, exams where few or no students pass or fail increase classification accuracy. Finally, allowing compensation increases classification accuracy.
\end{abstract}

Keywords: classification accuracy, misclassification, sets of exams, certification decisions

\section{Introduction}

Everyone agrees that high-stakes exams should be of sufficient quality. Quality of exams is usually studied in terms of validity and reliability for traditional standardized tests. The quality of exams that include performance assessments is generally studied in terms of authenticity (e.g. Gulikers, 2006) and the validity of the assessment (e.g. Linn, Baker, \& Dunbar, 1991). In contrast to traditional forms of assessment, performance assessments are not standardized tests. This implies that traditional reliability indices may not be suitable to apply to performance assessments (Clauser, 2000; Dochy, 2009). Kane (1996) points out that the precision of measurements is broader than just reliability. Classification accuracy provides an opportunity to quantify the quality of exams in a universal way, for both standardized tests and performance assessments. Especially in cases where reliability is difficult to compute, classification accuracy may give a quantitative measure of the quality of a certain exam. 
Classification accuracy is a measure of precision that may be more appropriate for performance assessments, yet also applicable to standardized tests. Classification accuracy is the degree of overlap between a decision based on the scores of an exam and the decision that would have been made on the basis of scores without any measurement error (Hambleton \& Novick, 1973).

There is no measurement, and thus no exam, without measurement error. Therefore, misclassifications occur wherever the decision based on the scores of an exam deviates from a decision based on error-free scores. There are two types of misclassifications: false positives or false negatives. False positives occur when students have a true classification below the set standard, but they receive an exam score above the standard. The reverse is false negatives: students that have a true classification above the set standard fail the exam. In all other casestrue classification above the standard passes the exam and true classification below the standard fails the exam - there is no misclassification. It must be noted that classification accuracy is not the same as classification consistency. Where classification accuracy compares the true classification of a student with the observed classification, classification consistency compares the classification in two (parallel) exams (see also Lee, 2008). Here, the interest is solely in classification accuracy.

In this chapter, the words standardized test, performance assessment, exam, and exam set all have a distinct meaning. A standardized test is any test that includes only questions, though they can be of any type: open-ended, multiple choice, and so on. A multiple choice test is a form of a standardized test. A performance assessment is defined as a form of testing in which the student is asked to perform a task that she could encounter in real life. Vocational education uses performance assessments to allow a student to display competency in their prospective jobs. The word exam is used whenever there is need for a general word for both performance assessments and standardized tests. The term exam set is used for any combination and number of exams. For instance, suppose a student must do three multiple choice tests and four performance assessments in order to receive a diploma. The seven exams together would be considered one exam set.

In essence, certification decisions and other high stakes exams tend to be dichotomous decisions. Either the student scores above or below the standard set for certification. One uses an exam to classify students above or below a set threshold, in order to hand out diplomas. It is very common to base diploma decisions on more than one exam. 
Usually an exam set is used that measures, as far as possible, all competencies and abilities involved. Generally, this seems like a good practice: more opportunities to observe a student give a better idea of true ability or competence. Furthermore, more measurements increase the reliability of the total measurement. Since the most important decision, the certification decision, is based on an exam set, it seems appropriate to compute the classification accuracy of that exam set instead of the classification accuracies of all separate exams.

Computing the classification accuracy of an exam set is not much different from computing the classification accuracy for a single exam. This chapter will use a method of computing classification accuracy for exam sets using simulation based on item response theory (IRT). The focus will be on how to increase classification accuracy of exam sets. In order to answer this question, three known influences on classification accuracy for single exams are studied: the shape of the ability distribution of the target population, the standards set for the exam set, and the compensation rules between the exams in the set.

Regarding the ability distribution of single exams, normalizing helps the simulation to more accurately estimate classification accuracy (Van Rijn, Béguin, \& Verstralen, 2009). An increase in classification accuracy is related to more symmetrical misclassifications (Holden \& Kelley, 2008), which is exactly what occurs when ability distributions are normalized.

With respect to the influence of standards on single exams, the following observations are made. Obviously, standards that are so extreme that they fall completely outside the population distribution lead to a classification accuracy of $100 \%$ (Lee, 2008). Furthermore, it is more difficult to correctly categorize students close to the standard than students far away from the standard (Martineau, 2007). Therefore, a graph of classification accuracy should show a dip around where the standard meets the average ability in the population (Lee, 2008).

Finally, the compensation rules are shown to have an effect on classification accuracy for exam sets (Van Rijn et al., 2009; Verstralen, 2009a). Both Van Rijn et al. (2009) and Verstralen (2009a) conclude that classification accuracy is increased when allowing some form of compensation. It is expected that this result is found for all other varied influences. Intuitively this makes sense, since allowing compensation essentially lengthens the separate exams into one long exam (Gatti \& Buckendahl, 2006). 
These three influences are studied in a simulation, using empirical data from a vocational education and training (VET) exam set as a starting point. Outcomes are expected not to differ from influences on single exams. In the next section of this chapter, the method of computing classification accuracy for the exam set is shown in short. Furthermore, the influences varied in the simulation study are discussed in more detail. The example section discusses the data source, the specific setup of the simulation study, and the results of this specific simulation. The following discussion section shows what the results imply for practitioners and discusses limitations of the study and future research.

\section{Method}

There are a few different ways of computing classification accuracy. Hambleton and Novick (1973) describe how to compute classification accuracy using two administrations of the same test. Swaminathan, Hambleton, and Algina (1974) gave a correction to this coefficient. In 1990, Huynh introduced a measure for class consistency for dichotomous items based on the Rasch model. Livingston and Lewis (1995) further elaborated on this measure, allowing for different scoring procedures. Schulz, Kolen, and Nicewander (1999) introduced the first real IRT model for estimating classification accuracy, though still only for dichotomous items. Wang, Kolen, and Harris (2000) extended this to a procedure for computing classification accuracy with polytomous IRT models. Verstralen (2009b) developed this into a method for computing classification accuracy for exam sets. This last method is used for the simulation and is explained further in the following paragraph.

\section{Simulation}

To measure the classification accuracy of a certain exam set, data need to be collected. Item parameters are estimated using an appropriate IRT model. This supposes that one exam measures

a certain ability or competency. Subsequently, the covariance matrix for the entire exam set can be estimated. Given this covariance matrix and the item parameters, a latent ability distribution for the population can be built. From either the given distribution or the estimated latent ability distribution, 5,000 true latent abilities are drawn. This enables the researcher to know the true ability and thus the true classification. In the case of an exam set, latent ability vectors are drawn, from which the true classification of the exam set is determined. 
Given the latent ability vectors and the item parameters, for each item the probability of a correct answer is computed. Using these probabilities, 5,000 item answer vectors are randomly drawn. From the answer vectors, the observed classification on the exam set is determined. Given the 5,000 observed and true classifications, the classification accuracy is simply determined by the percentage of correctly classified students.

\section{Varied Influences}

The ability distribution is determined through the IRT model as estimated. This distribution is supposed to approximate the population ability distribution. In the case of an exam set, the distribution is always a multivariate one. Three variations of the ability distribution were used in this chapter: the estimated empirical distribution, a centered distribution, and a standardized distribution. The empirical distribution was solely estimated from the data sample, described in the next section. Subsequently, the empirical distribution was centralized by subtracting the means of each of the exams; furthermore, the observations were divided by the standard deviation, giving a standard deviation of 1 . This leaves the distribution with all means 0 . Finally, the distribution was normalized.

Standards are the pre-specified cutoff point for a certain exam. There are two types of standards: norm-referenced and criterion referenced. Though these standards are set differently, they are always a known transformation of each other, given a fixed population. Therefore, this chapter varies norm-referenced standards. Bear in mind that if a norm-referenced criterion requires $60 \%$ of the points to be obtained, this is no indication that $60 \%$ of the students pass said exam. For each of the other varied influences, the standards are varied from $5 \%$ to $95 \%$ of the students passing a certain exam. The standards are kept the same for each of the exams in the exam set.

Compensation rules specify how the scores on separate exams should be combined into a single decision on the exam set. These rules prescribe how much compensation is allowed between the different exams. Compensation rules come in three different flavors: conjunctive, complementary, and compensatory (see also Van Rijn et al., 2009). Conjunctive rules allow no compensation; students should have a score higher than the standard on each exam. Complementary rules state that the student should score above the standard for a set number of exams. 
Compensatory rules allow complete compensation within an exam set, where the average score of the four exams should exceed a certain pre-specified standard. Conjunctive and complementary compensation rules are often used when there is a minimal level of ability or competence required. Compensatory rules, on the other hand, are often used when it can be justified that deficiencies in one competency are compensated with competency in other areas. There are many compensatory rules possible, depending on the number of exams and the leniency shown (Hambleton, Jaeger, Plake, \& Mills, 2000). In the simulation all three types are used, representing increasing leniency. The influence of allowing compensation between exams within an exam set is shown this way.

\section{Example}

The data sample was taken from vocational education and training (VET) in the Netherlands. The data used is described next in further detail, as well as the particulars of the simulation. Vocational education and training (VET) in the Netherlands is focused on building and assessing competencies (Gulikers, Bastiaens, \& Kirschner, 2004). Therefore, it utilizes performance assessments that find their base in a practical work-like setting. Usually, a few performance assessments are grouped together in an exam set for a certification decision. The exam set may or may not include standardized tests. Although the performance assessment is not the only type of assessment used in certification decisions, it is generally an important one; the performance assessment can constitute as much as $90 \%$ of an assessment program.

\section{Data Source}

Data were collected from a school for vocational education and training in the Netherlands. The exam set used was from business education and leads to a diploma in Sales Clerk Training (VET). In total, 188 students participated in the study (49\% female). This was deemed sufficient, since Martineau (2007) shows that around 200 observations are sufficient to use the computed classification accuracy as a reasonable point estimate of the true classification accuracy. Not every student had completed every exam yet. In VET, it is customary to hold the exam only with students that feel they have a good chance of passing the exam. Therefore, a booklet design where this could be incorporated was used. 
The exam set used consists of four performance assessments. Each performance assessment consists of a set of observations. These observations were taken as the items on which the IRT model for the simulation was based. The empirical competence distributions of both data sets were positively skewed and positively kurtosed, compared with the normal distribution (see also Figure 2).

\section{Setup of the Simulation}

Since the standards were set differently for each simulation, a way of consistently changing the grading system was devised. First, an estimation of the latent competence level was simulated. Based on that, the grades were divided over percentiles equidistant on both sides of the specified standard. For instance, where the normative standard was on $50 \%$ of the students passing, the cutoff percentages were $90,75,50,25$ and $10 \%$ passing. The students falling in between two given percentiles were given a grade. Some students scored exactly on the percentile. They got the benefit of the doubt and received the higher grade. This conforms to current practice in actual examinations.

The simulated observations were scored with six different grades from 0 to 5 . The grade 3 was taken as the passing grade. To examine how the standards set influence the amount of misclassification, a simulation was run for every standard between $5 \%$ passing and $95 \%$ passing, with $12.5 \%$ increments, except for the first and last increment (7.5\%). A simulation with these standards was run for every variation of ability distribution and compensation rule.

The data consist of polytomous items, as described above. Therefore, they were analyzed using a polytomous IRT model, the polytomous OPLM model (Verhelst, Glas, \& Verstralen, 1993). This model gives the item category response function, describing the response to item $i$, in which the probability of observing $X_{i}=j$ as a function of the ability vector, is given by

$$
\psi_{i j}(\theta)=\operatorname{Pr}\left(X_{i}=j \mid \theta\right)=\frac{\exp \left[a_{i}\left(j \theta-\sum_{g=1}^{j} \beta_{i g}\right)\right]}{1+\sum_{h=1}^{m_{i}} \exp \left[a_{i}\left(h \theta-\sum_{g=1}^{h} \beta_{i g}\right)\right]}, \quad\left(j=0, \ldots, m_{i}\right),
$$


where $\beta_{i g}, g=1, \ldots, m_{i}$ are the item response parameters and $a_{i}$ is the discrimination parameter for each item $i$.

Using this model, the item parameters per exam were estimated, creating a latent ability distribution under the assumption of a normally distributed ability for each exam. From the four separate ability distributions, a covariance matrix of the latent abilities was estimated. This multivariate normal latent ability distribution is the basis of the simulation. For each of the 5,000 replications, a true ability vector $\theta_{j}$ for replication $j$, was randomly drawn from the multivariate ability distribution. Given $\theta_{j}$, the true pass-fail status of each replication was determined.

Subsequently, the response process was imitated by generating a response vector $r_{j}$ for each replication $j$. Four randomly drawn numbers from a uniform distribution form a vector $u_{j}$, where for each element it holds that $r_{j}=m$ if $u_{j} \leq P\left(X_{i}=m \mid \theta_{j}\right)$. The generated response vectors $r_{j}$ are used in equation (1) to estimate ability vector $\hat{\theta}_{j}$, from which again a pass-fail status is gauged. Next, the vectors $\theta_{j}$ and $\hat{\theta}_{j}$ can be compared to compute the total amount of misclassification. Although the response vectors in the example each consist of four responses, only the pass-fail status of the entire exam set was compared. The pass-fail status was always subject to the compensation rule used in that set of replications.

Four different compensation rules were compared. One conjunctive rule, two complementary rules, and one compensatory decision rule were studied. Although the exam set should always average 3 or higher, there are many restrictions on the separate exams that can be set to influence the diploma decision. Using the conjunctive rule, students will only receive a diploma when they pass all their exams. Thus, every exam should be graded 3 or higher before a student receives a diploma. On the other side of the spectrum is the completely compensatory decision rule, where students obtain their diploma when they have an average on the exam set that is above or equal to the passing grade 3 . There are no additional restrictions for a pass on the exam set.

In between are two complementary rules, where students are allowed to compensate one or two deficiencies, respectively. A deficiency is defined as the number of points scored below the specified passing grade. A 2 is one deficiency, a 1 counts as two deficiencies, and a 0 counts as three deficiencies. With one deficiency allowed in the exam set, a 2 on one of the exams could be compensated with a 4 . Compensating a 1 with a 5 is not allowed. 
When two compensations are allowed, both a 1 and a 2 can be compensated, given high enough grades on the other exams. In this specific example, the complementary rule that allows three deficiencies is equal to the compensatory rule.

\section{Results}

This section reviews the results of the simulations. Knowing what impacts classification accuracy helps to make decisions regarding the examination process. It is important to remember that the classification accuracy reflects the accuracy of the certification decision. First, the influence of the distribution is discussed. Second, the impact of changing normative standards is introduced. Finally, the results regarding different decision rules are discussed.

It has been shown that the shape of the ability distribution influences classification accuracy of single exams (Holden \& Kelley, 2008). The simulations demonstrate that a similar influence is found for exam sets, as shown in Figure 1. When comparing the three rows of the figure, it becomes apparent that the more the ability distribution of the population follows the assumptions of the model used to compute the classification accuracy, the higher the accuracy becomes. There is a small increase in average classification accuracy from the empirical ability distribution (95\%) to the normalized and standardized distributions (both on average 96\%). However, visually it is immediately apparent that this is a very consistent result over all the other conditions. This implies both that the model is better in estimating the classification accuracy and that when the model fits the ability distribution of the population, fewer misclassifications are made. These results were expected. 


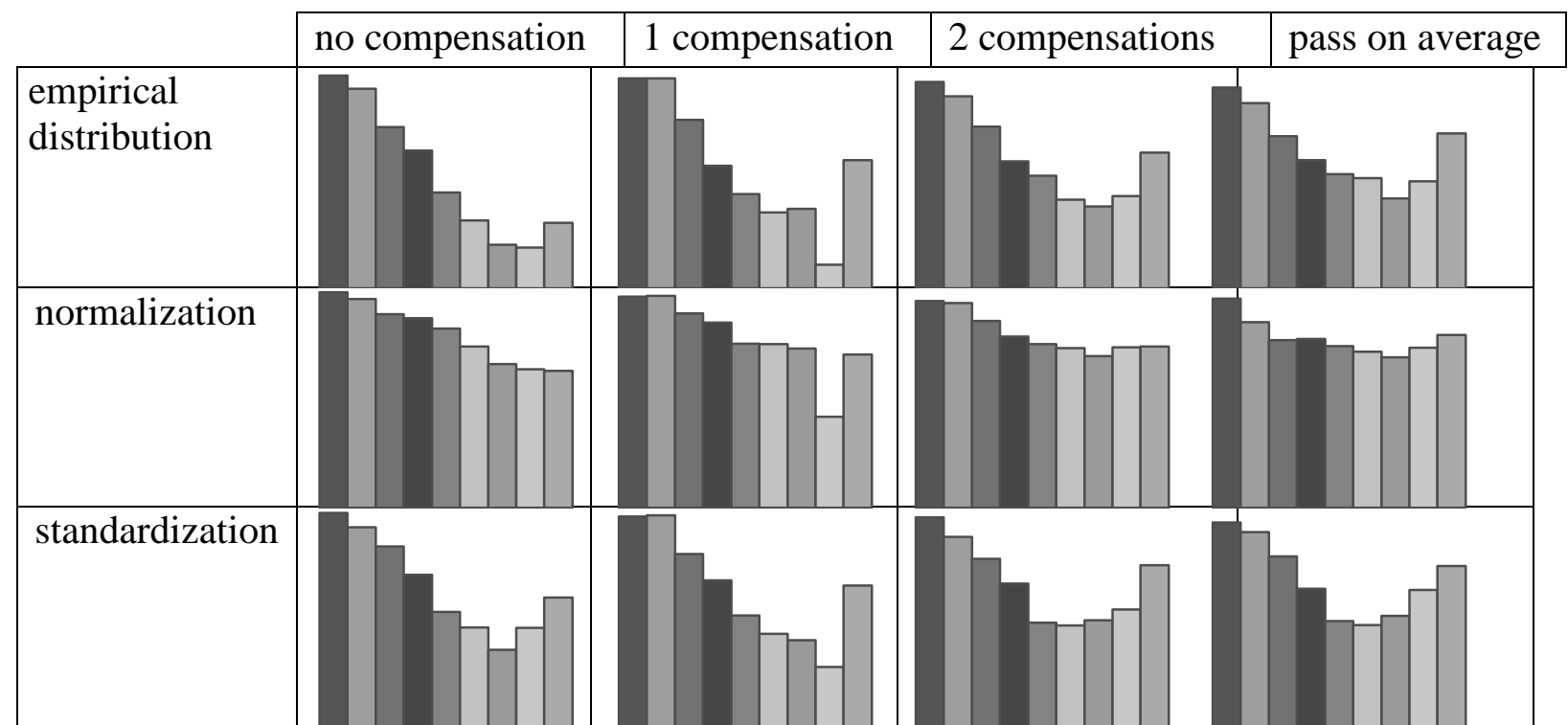

Note: The height of the columns corresponds to the percentages correctly classified students. The scale of the vertical axis is from $88 \%$ to $100 \%$ accuracy. Each bar plot contains all standards.

Figure 1 Classification accuracy per condition for all standards

A second part of the study looked at the influence of the standards of the exams on classification accuracy of the exam set. In Figure 1, the effect of the standards is visible by inspecting the 12 single graphs. From left to right, each graph shows the standards from 5\% obtaining a diploma to $95 \%$ obtaining a diploma. Within each graph, the bars show a line with a dip. The dip consistently occurs around the point where the top of the ability distribution of the target population is. This is as expected. 


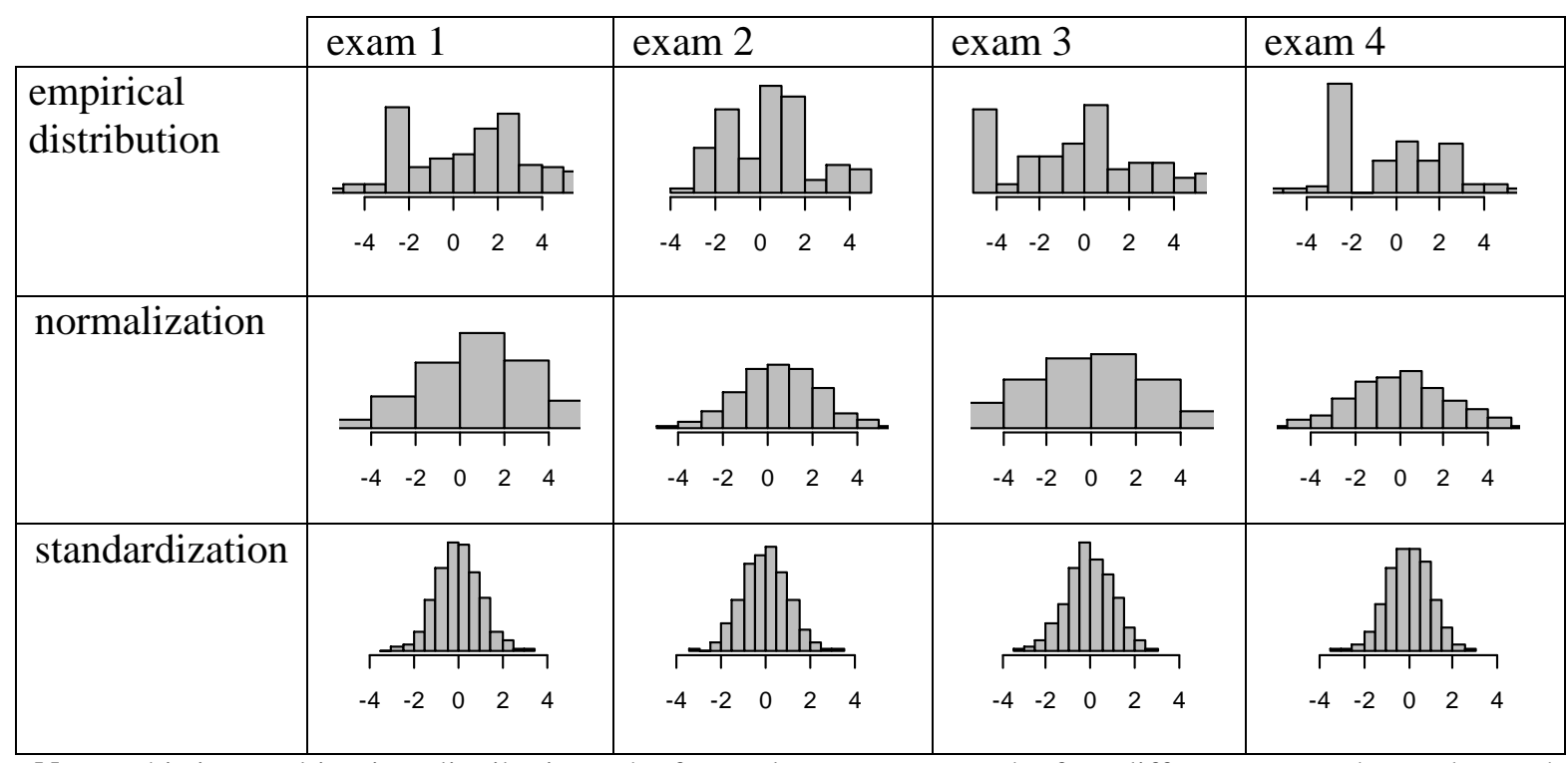

Note: This is a multivariate distribution. The four columns represent the four different exams that make up the exam set used. Rows represent the different distributions. From top to bottom: empirical distribution, normalized distribution, and standardized distribution.

Figure 2 Information on the ability distribution for all distributions

As can be seen in Figure 2, the empirical distribution shows an irregular pattern, resulting in an irregular multivariate distribution. The normalized distribution shows more evenly spread, but still slightly skewed, distributions. This translates into a flatter classification accuracy pattern than for the empirical distribution. The larger dip in the classification accuracy pattern of the standardized distribution coincides with the observed standardized distribution, which is more kurtosed than the normalized distribution.

The highest accuracy is found nearer the extreme standards (where everyone fails every exam or everyone passes every exam) and the lowest accuracy at the top of the ability distribution. Extreme standards, where either nearly all students pass or nearly all fail, are usually not desirable in educational settings. In certain settings, for instance when selecting a top-ten group of students or candidates, extreme standards may apply.

There are two reasons for the coinciding of the dip and the ability distribution. Especially in VET, it is well known what students should know or be able to do for each exam. This undoubtedly leads to "teaching to the test," a phenomenon where students and teachers work toward the level the test asks, the standards (Popham, 2001). Some might say that this is a problem. However, when the standards are set at an adequate level, this is not necessarily true. 
The result should be adequately trained starters and if the standards of the exams are adequate for that goal, it might even be desirable to teach to the test. Should institutes for learning or examination desire a higher classification accuracy, they may consider setting a level of learning higher than the level of examination.

The study also investigates the influence of compensation rules. Figure 1 shows that an exam set in which compensation is allowed leads to higher classification accuracy. When looking from left to right, all classification accuracies show increased classification accuracy, with the increasing freedom of compensation. The third row shows this best. In the last graph, the classification accuracy for each standard is comparable. For standards below $50 \%$ of students passing, the compensation rules have a less pronounced effect. This seems due to the ability distribution. The classification accuracy for different compensation rules converges on both sides of the dip. Since a ceiling effect occurs, the classification accuracy does not converge on the higher standards. It must be brought to mind that the compensation is between exams, not within exams. The condition "no compensation" means that each exam must have been finished with a passing grade, not necessarily with a perfect score.

Table 1 Classification accuracy in percentage of correctly classified students

\begin{tabular}{llll}
\hline & & real standard & average of all standards \\
\hline empirical & no compensation & $88 \%$ & $94 \%$ \\
distribution & 1 compensation & $91 \%$ & $95 \%$ \\
& 2 compensations & $92 \%$ & $95 \%$ \\
& pass on average & $93 \%$ & $96 \%$ \\
\hline normalisation & no compensation & $97 \%$ & $98 \%$ \\
& 1 compensation & $96 \%$ & $97 \%$ \\
& 2 compensations & $96 \%$ & $98 \%$ \\
& pass on average & $97 \%$ & $97 \%$ \\
\hline standardisation & no compensation & $96 \%$ & $96 \%$ \\
& 1 compensation & $96 \%$ & $96 \%$ \\
& 2 compensations & $96 \%$ & $96 \%$ \\
& pass on average & $98 \%$ & $96 \%$ \\
\hline
\end{tabular}

When just looking at the empirical distribution, set at the currently used standards (see Table 1), the influence of compensation shows that each step of compensation adds some accuracy. No compensation leads to a classification accuracy of $88 \%$. 
Obviously, this leaves $12 \%$ of the students to be misclassified. Allowing some compensation increases classification accuracy for this exam set (1 compensation: 91\%; 2 compensations: $92 \%)$.

However, the highest classification accuracy is achieved using full compensation (pass on average: 93\%). This implies that nearly half the misclassified students of the no compensation scenario are correctly classified under full compensation. This could be a reason to implement a higher level of compensation. It is interesting also to note that the biggest increase in classification accuracy is found going from no compensation at all to at least some compensation (difference of $91 \%-88 \%=3 \%$ ). Depending on the amount of students partaking in the exams, it may be worthwhile to consider at least partial compensation for the exam set. These results had been anticipated as well, since allowing compensation essentially lengthens the exams within the exam set and therefore it is expected that the exam set is better able to classify the students.

\section{Discussion}

Most research focuses on the quality of individual exams. However, the important decisions are usually based on an exam set. Therefore, it seems more appropriate to focus on the quality of exam sets. Much has been written about classification accuracy of single exams; there is literature about several ways of computing classification accuracy and there is literature regarding the influences on this classification accuracy.

A few well-known influences are the shape of the ability distribution of the measured ability in the target population, the standard that has been set for the exam, and the allowance of compensation within the exam. It was shown how the classification accuracy of exam sets is influenced by ability distribution, standards, and compensation. This was done using an example from vocational education and training (VET). The classification accuracy for an exam set is computed using item response theory (IRT) simulation. The outcomes indicate that classification accuracy for exam sets is influenced in a way similar to the classification accuracy for single exams. Of course, this stays partially contingent on the quality and number of the exams that together make up the exam set. 
Classification accuracy is high when all tests from an exam set have equal and standardized ability distributions. To some extent, schools may exert some influence over the ability distribution. However, researchers or test developers do not have this possibility. Nevertheless, this does imply that concepts that are stable and normally distributed may be tested with higher classification accuracy. Test developers may want to research the concepts they plan to test beforehand, in order to assess whether they can accurately test them.

It is neither always possible nor always desirable to create exam sets that fit the ability distribution of the target population. In some cases, the target population is unknown. Other constraints may be time and money to obtain a distribution. Furthermore, ability distributions may shift over time. Even when the target population and its ability distribution are known, it may change in the time between measuring the distribution and taking the exams. Finally, it is not expected that the ability distribution is completely normal, since most exams in VET are designed with a ceiling effect in mind.

Furthermore, extreme standards (where few or no students pass or fail an exam) increase classification accuracy. In practice, however, it is not common to test far below or above the average ability of the target population. This is especially true in educational settings. Furthermore, developing exam sets that are designed to test outside the ability distribution of the target population do not yield much information. In certain situations, one may still decide to develop such an exam set. For instance, one may want to ensure that all students have a certain basic proficiency, where it is expected that all students easily surpass this proficiency. Measurement is likely to be more accurate if the question of how proficient students are is subordinate to the question of whether all students are proficient enough.

Finally, allowing compensation increases classification accuracy. Compensation, either within a single exam or encompassing the entire exam set, is a property of the exam that researchers or test developers nearly always have an influence on. There are various reasons why one may want to allow or disallow compensation. This discussion limits itself to reasons for allowing and disallowing compensation in exam sets. Reasons for allowing compensation include increasing classification accuracy. Furthermore, when individual exams are short, and they tend to be in VET, it seems reasonable to partially negate the effects of measurement error with the allowance of compensation between exams. Moreover, intuitively it may feel unfair for a good student to be denied a diploma should she fail just one exam. 
Nevertheless, there are arguments in favor of conjunctive exam sets. In some vocations there are at least certain parts of the examination that should be passed in any condition. A nurse that is incapable of inserting an IV needle should not be allowed to practice. In addition, conjunctive measurement may be the fastest, and thus cheapest, way of examination. Besides, it may be the easiest rule to explain to the students, giving them the required insight in their assessment.

On the whole, it is advisable to compute classification accuracy for exam sets. It gives the researcher or test developer insight into the quality of the exam set, rather than just the separate exams. One especially gains insight into how many students are disadvantaged by the method of examination. Moreover, when the quality of the separate exams is difficult to assess, classification accuracy is an elegant measure for gauging the quality of the examination.

On the other hand, computing classification accuracy is rather expensive. It costs time and requires a fair amount of skill on the part of the researcher or test developer, not to mention the lack of easily accessible software. It may only be worth investing the resources when stakes are high, for instance in the case of certification exam sets, or when the quality of the exam set is highly disputed. Of course, when the resources are readily available, classification accuracy sure seems a great measure to add to the findings on exam set quality.

\section{References}

Clauser, B. (2000). Recurrent Issues and Recent Advances in Scoring Performance Assessments. Applied Psychological Measurement, 24(4), 310-324.

Dochy, F. (2009). The Edumetric Quality of New Modes of Assessment: Some Issues and Prospects. In G. Joughin (Ed.), Assessment, Learning and Judgement in Higher Education (pp. 85-114). Springer Science.

Gatti, G. G., \& Buckendahl, C. W. (2006). On Correctly Classifying Examinees. In Annual Meeting of the American Educational Research Association (San Francisco, CA). Retrieved April 26, 2011 from http://www.unl.edu/buros/biaco/pdf/pres06gatti01.pdf.

Gulikers, J. T. M., Bastiaens, T. J., \& Kirschner, P. A. (2004). A Five-Dimensional Framework for Authentic Assessment. Educational Technology Research and Development, 52(3), $67-85$. 
Hambleton, R. K., Jaeger, R. M., Plake, B. S., \& Mills, C. (2000). Setting Performance Standards on Complex Educational Assessments. Applied Psychological Measurement, 24(4), 355366.

Hambleton, R., \& Novick, M. (1973). Toward an Integration of Theory and Method for Criterion-Referenced Tests. Journal of Educational Measurement, 10(3), 159-170.

Holden, J. E., \& Kelley, K. (2008). Effects of Misclassified Data on Two Methods of Classification Analysis: A Monte Carlo Simulation Study. Paper presented at the Annual Meeting of the American Educational Research Association, New York, NY.

Huynh, H. (1990). Computation and Statistical Inference for Decision Consistency Indexes Based on the Rasch Model. Journal of Educational Statistics, 15, 353-368.

Kane, M. (1996). The Precision of Measurements. Applied Measurement in Education, 9(4), 355379.

Lee, W. C. (2008). Classification Consistency and Accuracy for Complex Assessments Using Item Response Theory. Iowa City: Center for Advanced Studies in Measurement and Assessment.

Linn, R. L., Baker, E. L., \& Dunbar, S. B. (1991). Complex, Performance-Based Assessment: Expectations and Validation Criteria. Educational Researcher, 20(8), 15-21.

Livingston, S. A., \& Lewis, C. (1995). Estimating the Consistency and Accuracy of Classifications Based on Test Scores. Journal of Educational Measurement, 32, 179-197.

Martineau, J. A. (2007). An Expansion and Practical Evaluation of Expected Classification Accuracy. Applied Psychological Measurement, 31(3), 181-194.

Popham, W. J. (2001). Teaching to the test. Educational Leadership, 58(6), 16-20.

Schulz, E. M., Kolen, M. J., \& Nicewander, W. A. (1999). A Rationale for Defining Achievement Levels Using IRT-Estimated Domain Scores. Applied Psychological Measurement, 23, 347-362.

Swaminathan, H., Hambleton, R. K., \& Algina, J. (1974). Reliability of Criterion-Referenced Tests: A Decision-Theoretic Formulation. Journal of Educational Measurement, 11,263268.

Van Rijn, P., Béguin, A., \& Verstralen, H. (2009). Zakken of Slagen? De Nauwkeurigheid van Examenuitslagen in het Voortgezet Onderwijs. (Pass or Fail? The Accuracy of Exam Results in Secondary Education) Pedagogische Studiën, 86, 185-195. 
Verhelst, N. D., Glas, C. A. W., \& Verstralen, H. H. F. M. (1993). OPLM: One parameter logistic model. Computer program and manual. Arnhem: Cito.

Verstralen, H. (2009a). Quality of Certification Decisions. Arnhem: Cito.

Verstralen, H. (2009b). Accuracy of Exams: CTT and IRT Compared. Arnhem: Cito 


\title{
Chapter 11
}

\section{Computerized Classification Testing and Its Relationship to the Testing Goal}

\section{Maaike M. van Groen}

\begin{abstract}
Assessment can serve different goals. If the aim of testing is to classify respondents into one of multiple levels instead of obtaining a precise estimate of the respondent's ability, computerized classification testing can be used. This type of testing requires algorithms for item selection and making the classification decision. The result of the test administration is provided in a report about the decision with sometimes additional feedback. The design of all these components of the test should be in line with the testing goal. Several goals have been defined for assessment which make a judgment about: pupils, the learning process, groups of students and schools, and the quality of education. The possibilities for use of computerized classification testing for different testing goals are investigated in the current paper.
\end{abstract}

Keywords: computerized classification testing, testing goals, test design

\section{Introduction}

Assessment can have different goals. In some testing situations, the aim is to classify respondents into one of multiple levels instead of making a precise estimate of the respondent's ability. This should be achieved by administering as few items as possible while maximizing the number of correct classifications. Computerized classification testing (CCT) is an approach that can be used for finding a balance between the number of items and the level of confidence in the correctness of the decision (Bartroff, Finkelman, \& Lai, 2008). According to Thompson (2009), computerized classification tests assign an examinee into one of two or more mutually exclusive categories along the ability scale. In the current paper, this definition is further limited to tests based on item pools that have been scaled using modern psychometric methods.

One part of the procedure determines which items have to be selected. Another part of the CCT procedure determines whether testing can be stopped because enough confidence has been gained in making the decision or that an additional item has to be administered. 
The classification method as well as the item selection method have to be in line with the testing goal and the report and feedback that have to be provided after testing has been finished. This is illustrated in Figure 1. Based on the testing goal, a method for reporting the classification and the type of feedback can be determined. The testing goal also partly determines which classification method can be used and how it should be implemented. The goal influences the selection of the item as well. The way in which results can be reported and feedback can be provided is determined by the classification method as well as the item selection method. Ideally, these methods should be designed so the desired report and feedback can be provided afterwards. A test developer should always keep in mind that the goals, methods, and report should be synchronized with each other.

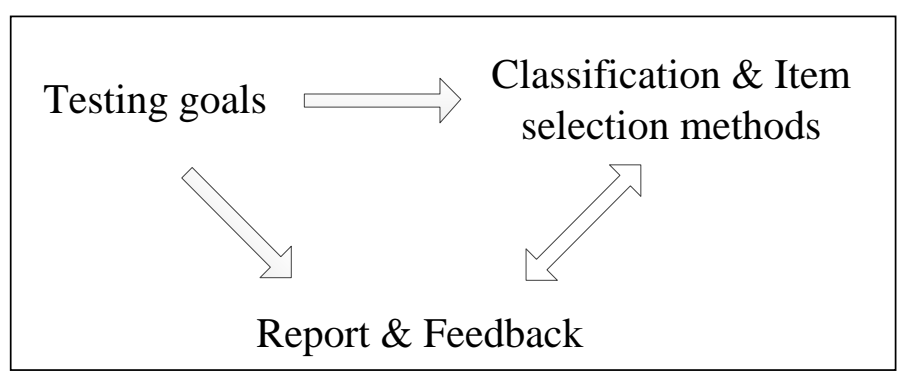

Figure 1 Testing goals and CCT components

\section{Testing Goals and Computerized Classification Testing}

In the previous section, attention was paid to testing goals. However, which goals testing can have was not described. The implications for classification and item selection methods and report and feedback have been mentioned only briefly. In this section, first, testing goals and computerized classification testing are described. The possibility of using computerized classification testing for specific testing goals is then described. In the last section, the implications of testing goals for designing computerized classification tests are investigated.

Testing can serve different goals. One taxonomy of testing goals is provided by Sanders (2011). He divides testing goals into: 
- Assessment for making a judgment about students

- Assessment for making a judgment about the learning process

- Assessment for making a judgment about groups of students and schools

- Assessment for making a judgment about the quality of education.

A second distinction can be made regarding the importance of the consequences of testing because the importance of the test has a major influence on test design. Stobart (2008) defines a high-stakes test as having substantial consequences for some or all of the parties involved. A third distinction can be made regarding the type of assessment: assessment for learning or assessment of learning. Assessment for learning is used as a tool for supporting the learning of pupils by providing guidance for the instructional process. Assessment of learning includes all tests that measure knowledge after a period of instruction to assess whether the required knowledge level has been reached or not. Since the testing goal, the importance of testing, and the type of assessment are closely related to each other, they are described together.

\section{Assessment for Making a Judgment about Pupils}

Assessment for making a judgment about students can be subdivided into four subgoals:

\section{- Selection}

- Classification

- Placement

- Certification

Sanders (2011) explains that selection takes place if not all the students who want to enroll in a program or study can be admitted. Based on the selection decision, a fixed number of students are admitted to an educational program. A selection decision can be made based on a specially designed test, for example, the Law School Admission Test for admission to law school in the United States or based on a test with a more general goal. An example of the latter are the final examinations for secondary education in the Netherlands used for selecting students for admittance to medical school. 
Based on the classification decision in a classification test, a different educational program is offered to the student that will lead to a different diploma (Sanders, 2011). The final test for Dutch primary education (Cito, 2012) is one of the instruments that can be used for deciding the level of secondary education a child will attend in addition to the teacher's advice and the parents' ideas. If placement is the testing goal, the student will be placed in a different educational program, but the final diploma will be the same. An example is an entrance driving test that is used for selecting students for a short driving course who will be able to pass the driving examination after only a limited number of driving lessons. Those who are expected to need more lessons will be selected for a longer driving course. The last testing goal is certification. Certification tests are used in situations in which a final judgment has to be made regarding the student's level in order to receive a certificate or a diploma. Well-known examples of such tests are the final examinations in secondary education in the Netherlands. These testing goals have in common that they are a form of assessment of learning and that they can all be seen as a form of high-stakes testing. The goal is to make a summative judgment of the student's knowledge.

\section{Assessment for Making a Judgment about the Learning Process}

In assessments in which a judgment is made regarding the student's learning process, the goal is to obtain information that can be used in the instructional process (Sanders, 2011). This can be seen as assessment for learning. Using such a test, the teacher will be able to adapt his or her instruction to increase the students' knowledge and skills. Diagnostic tests also serve this goal of testing. If diagnostic testing is the goal of testing, the interested reader is referred to Rupp, Templin, and Henson (2010). Also tests like the Mathgarden (www.mathsgarden.com), a serious game for primary education arithmetic, and simulation-based learning in aviation can be seen as making judgments about the learning process of the student. Sanders (2011) points out that the distinctions between testing and instruction will become blurred in these tests. Tests that serve this goal have a major impact on the teaching methodology but usually have only indirect impact on the pupils themselves. 


\section{Assessment for Making a Judgment about Groups of Students and Schools}

Assessments for making a judgment about groups of students and schools take place if the test results of individual students are aggregated to get information about the group or the school (Sanders, 2011). Assessment for making a judgment about groups of students and school can serve different purposes. If the focus is on improvement of the learning of the group of students, it can be seen as an assessment for learning. If the focus is on accountability for the results of the group or the school, it can be seen as assessment of learning. An example of the former is the situation in which small groups within the class are arranged based on their achievements on a test in order to provide different instruction to each group. An example of the latter is the use of test scores for giving the Inspectorate insight into the quality of the school. The consequences in this situation are highest for the school instead of for the individual pupil (Stobart, 2008).

\section{Assessment for Making a Judgment about the Quality of Education}

The last goal of testing is assessment for making a judgment about the quality of education. In these studies, the goal is to measure the quality of the education in a nation or to compare educational systems in different nations (Sanders, 2011). Such studies, such as PPON and PISA, provide policymakers, the Inspectorate, developers of instructional and assessment material, and so on, insight into the current level of pupils in the nation. Based on the findings, adjustments in policy and materials can be made. Since test results are not used for improvement of education on the individual level, these tests can be seen as assessment of learning. The stakes in these tests are primarily on the national level.

\section{Computerized Classification Testing for Different Testing Goals}

Computerized classification testing can be used in many different situations. In this section the use of CCT is explored for the testing goals as defined by Sanders (2011). The efficiency and effectiveness of CCT is compared to linear testing and computerized adaptive testing for those goals. In computerized adaptive testing the goal is to obtain a precise estimate of the respondent's ability level on a continuous scale instead of making a classification decision into one of multiple mutually exclusive categories. But first, some additional information is provided about computerized classification testing. 


\section{Computerized Classification Testing}

CCT requires two algorithms. The first determines when a classification decision can be made. The second determines which item has to be administered next. Several methods exist for making a classification, such as the sequential probability ratio test (Wald, 1947/1973; Reckase, 1983; Eggen, 1999) and the ability confidence interval method (Weiss \& Kingsbury, 1984). The majority of these methods can classify respondents into two levels, but some can also classify respondents into multiple groups. Commonly used item selection methods such as maximization of information at the cutting point and maximization at the current ability estimate can be used if classification into two groups is desired (Eggen, 1999), but some methods can also be used if a classification into multiple levels is required (Eggen \& Straetmans, 2000; Van Groen, Eggen, \& Veldkamp, 2012).

\section{Assessment for Making a Judgment about Pupils}

Computerized classification testing was originally designed for dividing respondents into different groups. If the assessment is used for making a judgment about pupils, computerized classification testing is one of the most efficient methods. Because decisions about pupils have a major impact on students, a high level of accuracy is desired. In CCT, accuracy is maximized while test length is minimized. Depending on the precise testing goal, more or less accuracy is required. If the goal is classification or certification, accuracy is extremely important because of the stakes for the student. CCT cannot be used if selection of students is the goal of the assessment because CCT requires a fixed cutting point instead of a flexible cutting point. When selection takes place, the cutting point is set at the value that results in the specified number of students who pass.

Linear testing can be used for making classification decisions, but many more items are required to make the classification decision as accurate as in CCT. Computerized adaptive testing (CAT) also requires more items than necessary because in CAT precise estimates have to be acquired at all points on the ability scale. In CCT, however, precision is required only on one or more points on the ability scale if a classification decision has to be made. CAT is well suited for making selection decisions because the cutting point can be set at every point on the scale after all tests have been administered. 


\section{Assessment for Making a Judgment about the Learning Process}

If assessment for making a judgment about the learning process is the goal, computerized classification testing can be used if a precise ability estimate is not required. If a classification decision on subdomains, such as multiplication, division, and so on, is sufficient, CCT can be used; otherwise, CAT or linear testing has to be used. If CAT or linear testing is used, more items will be required for obtaining information about the student's level. Different models can be used in CAT and linear testing that have been designed for diagnostic testing especially (Rupp, Templin, \& Henson, 2010).

In assessment for making a judgment about the learning process, the idea is to gather information within a rather short time and use the test results to adapt the instruction to the students. This implies that only a limited number of items will be available for making the classification decision and accuracy is not the most important goal. If diagnostic information has to be gathered on several subdomains, a limited number of items will be available per subdomain, and per subdomain a classification decision has to be made.

\section{Assessment for Making a Judgment about Groups of Students and Schools}

If a judgment has to be made about groups of students or schools in the context of assessment for learning, the same conditions apply as for making judgments about the learning process for individual students. In both situations, the ultimate goal is to adapt the instruction the teacher provides to the students' knowledge level. The difference is in the focus on the judgment groups and schools instead of individual students.

If a judgment has to be made about groups of students or schools in the context of assessment of learning, computerized classification testing can be used if a classification decision suffices. If different cutting points have been set for schools due to different student characteristics, this is also possible. If more information is required than CCT can provide, CAT or linear testing has to be used.

\section{Assessment for Making a Judgment about the Quality of Education}

If assessment for making a judgment about the quality of education is the goal of testing, whether CCT can be used depends on the specific results policymakers want to be measured. If the goal is to investigate whether the required subjects are mastered by pupils, CCT can be used. 
In situations in which the effect of a reform has to be investigated, the policymakers are interested in differences in ability before and after the reform. CAT and linear testing are better suited for evaluation of reforms. In the first situation, the stakes are at the national level instead of at the student level.

\section{Designing Computerized Classification Tests for Different Testing Goals}

The relationship between testing goals and components of a computerized classification test were described in Figure 1. The classification method, item selection method, report, and feedback should all be designed so that they are in line with the testing goal. In this section, the four design components are investigated for the different testing goals.

\section{Assessment for Making a Judgment about Pupils}

In a computerized classification test for making a judgment about pupils, traditional CCT classification methods can be used for making the decision whether to classify into a certain level or to continue testing. An algorithm can be selected based on the number of cutting points needed for the test. The focus of the item selection method should be on obtaining the most information as quickly as possible to be able to stop testing after as few items as possible. If one cutting point is used, information can be maximized at the cutting point (Eggen, 1999). If multiple cutting points are used, an algorithm that takes this into account has to be used (Eggen \& Straetmans, 2000; Van Groen, Eggen, \& Velkamp, 2012). Using simulations, optimal settings for the classification method and item selection method can be determined. The report in a CCT for making a judgment about pupils can be simple. Reporting the actual decision often suffices. Specific feedback is not needed in these situations because the decision is all that matters.

\section{Assessment for Making a Judgment about the Learning Process}

If a computerized classification test is used for making a judgment about the learning process, the classification method has to include one decision per subdomain. This implies that per subdomain a classification has to be made about mastering the subdomain or not. It is also possible to include multiple levels in the classification method per subdomain. The number of items that have to be administered before stopping the test is strongly related to the number of subdomains and the number of cutting points per subdomain. 
The design of the classification method should be in line with the specific theories behind the topic and should conform to the level of specificity a teacher needs to adapt the instruction to the student's level.

The item selection method should select items for the subdomain for which a classification decision has to be made. This implies that some kind of content control is required within the item selection method. To make decisions with as much information as possible, items should be selected that maximize information at the cutting point that is of interest. If items provide information regarding several subdomains, developing a special item selection method for the test can be more efficient. Simulation studies provide insight into the efficiency and side effects of different item selection methods.

The report should provide the information a teacher needs to adapt the instruction. Per subdomain the classification decision has to be provided. If available, specific feedback for improving the instruction can be given such as references to relevant exercises and instruction material. In a second screen, information could be provided per domain regarding the items that the student answered correctly or incorrectly. Additional feedback can be provided about the types of mistakes a student makes when answering the item. The report and the feedback should be well structured and easy to comprehend; if not, the teacher will look only at the classifications.

\section{Assessment for Making a Judgment about Groups of Students and Schools}

If a computerized classification test is used for judging groups of students and schools in the context of assessment for learning, the basic guidelines for CCT for judging the learning process can be followed. Differences should appear in the way the report is presented after the test is administered. The focus should be on groups of students or on the school. Aggregated results can be presented per subdomain with feedback on how instruction could be improved. Instead of providing information about individual students, the number of students that have gathered not enough knowledge about a subdomain could be presented. In addition, clusters of students with similar profiles based on the classifications can be provided. The teacher can provide instruction to the groups of students based on the profiles. If a computerized classification test is used for judging groups of students and schools in the context of assessment of learning, the classification method should be directed toward making a classification after as few items as possible. 
The item selection method should also be directed toward gathering evidence for making the classification decision as quickly as possible. The report can be very basic. The percentage or number of students who pass the test should be reported. Feedback is not needed in this situation because the goal is to provide information for accountability purposes only.

\section{Assessment for Making a Judgment about the Quality of Education}

If a CCT is used for judgment the quality of education, the design of the components of the CCT can be comparable to the design for accountability. The difference between the two goals is primarily visible in the report. Instead of aggregation to the group or school level, aggregation should be at the national level. Specific feedback is not necessary.

\section{Discussion}

Computerized classification testing can be used in many testing situations in which students have to be classified into groups who have gathered knowledge at a certain level. By including subdomains in the classification method, it becomes possible to use CCT in more situations than often realized. The main reasons for not using CCT include requirements for giving scores at a continuous scale and possible objections against computerized testing.

Test developers should always keep the goal of their test in mind when designing the test. This is not different from traditional paper-and-pencil tests or computerized adaptive tests, but only limited theoretical work has been done for computerized classification testing. During the construction phase, the test developer should always keep Figure 1 in mind: the testing goals

define the requirements for the classification method, item selection method, report, and feedback. The classification and item selection methods restrict the information and feedback that can be reported, which implies that these four components have to be designed concurrently. 


\section{References}

Bartroff, J., Finkelman, M. D., \& Lai, T. L. (2008). Modern sequential analysis and its applications to computerized adaptive testing. Psychometrika, 73, 473-486. doi: 10.1007/s11336-007-9053-9

Cito. (2012). Eindtoets Basisonderwijs [Final test primary education]. Arnhem: Cito BV.

Eggen, T. J. H. M. (1999). Item selection in adaptive testing with the sequential probability ratio test. Applied Psychological Measurement, 23, 249-261. doi: $10.1177 / 01466219922031365$

Eggen, T. J. H. M. \& Straetmans, G. J. J. M. (2000). Computerized adaptive testing for classifying examinees into three categories. Educational and Psychological Measurement, 60, 713-734. doi: 10.1177/00131640021970862

Groen, M. M. van, Eggen, T. J. H M., \& Veldkamp, B. P. (Unpublished). Item Selection Methods Based on Multiple Objective Approaches for Classification of Respondents into Multiple Levels.

Reckase, M. D. (1983). A procedure for decision making using tailored testing. In Weiss, D. J. (Ed.), New horizons in testing: latent trait theory and computerized adaptive testing (pp. 237-254). New York, NY: Academic Press.

Rupp, A. A., Templin, J., \& Henson, R. A. (2010). Diagnostic measurement: Theory, methods, and applications. New York, NY: The Guilford Press.

Sanders, P. (2011). Het doel van toetsen [The goal of testing] In Sanders, P. (Ed.), Toetsen op school [Testing in schools] (pp. 9-20). Arnhem: Stichting Cito Instituut voor Toetsontwikkeling.

Stobart, G. (2008) Testing Times: The uses and abuses of assessment. London: Routledge.

Thompson, N. A. (2009). Item selection in computerized classification testing. Educational and Psychological Measurement, 69, 778-793. doi: 10.1177/0013164408324460

Wald, A. (1947/1973). Sequential analysis. New York, NY: Dover Publications, Inc.

Weiss, D. J. \& Kingsbury, G. G. (1984). Application of computerized adaptive testing to educational problems. Journal of Educational Measurement, 21, 4, 361-375. doi: $10.1111 /$ j.1745-3984.1984.tb01040.x 


\title{
Chapter 12
}

\section{An Overview of Innovative Computer-Based Testing}

\author{
Sebastiaan de Klerk
}

\begin{abstract}
Driven by the technological revolution, computer-based testing (CBT) has witnessed an explosive rise the last decades, in both psychological and educational assessment. Many paperand-pencil tests now have a computer-based equivalent. Innovations in CBT are almost innumerable, and innovative and new CBTs continue to emerge on a very regular basis. Innovations in CBT may best be described along a continuum of several dimensions. Parshall, Spray, Kalohn, and Davey (2002) describe five innovation dimensions in which CBTs can differ in their level of innovativeness: item format, response action, media inclusion, level of interactivity, and scoring method. This chapter provides a detailed description of the five innovation dimensions, including case examples. Furthermore, an overview of opportunities, risks, and future research will be given.
\end{abstract}

Keywords: computer-based testing, dimensions of innovation, opportunities and risks, future research

\section{Introduction}

The availability and utilization of personal computers has been growing explosively since the 1980s, and will continue to do so in the coming decades. The educational system has not been oblivious to the explosive rise of PCs and technology in general. For example, the development of high-speed scanners, or Optical Mark Recognition (OMR), halfway through the 1930s of the $20^{\text {th }}$ century introduced the possibility of automatically scoring multiple-choice tests. More recently, during the late 1970s, the first computer-delivered multiple-choice tests emerged, and computer-based testing (CBT) was born. Further improvements and cost reductions in technology made the application of large-scale, high-stake CBTs during the 1990s possible. Present advances in technology continue to drive innovations in CBT, and new CBTs are being designed on a regular basis by a whole range of educational institutions. 
Nowadays, test developers can incorporate multimedia elements into their CBTs, and they can develop innovative item types, all under the continuing influence of technology improvements. This chapter provides an overview of innovations in CBT.

Because innovations in CBT are (almost) innumerable, and continue to emerge in many different forms, a dichotomous categorization of CBTs as innovative versus non-innovative is not possible. More specifically, however, innovation in CBTs may be seen as a continuum along several dimensions. For instance, some CBTs may be highly innovative (scoring innovativeness on multiple dimensions), while other CBTs are less innovative (scoring innovativeness on only one dimension). Inclusion of media (e.g., video, animation, or pictures), test format (e.g., adaptive), item format (e.g., drag- and drop-, matrix-, or ranking and sequencing questions), and construct measurement (e.g., skills or competencies) are all attributes upon which the innovativeness of a CBT can be determined. In general, using PCs or technology to develop creative ways of assessing test takers or to measure constructs that were previously impossible to measure is the most important dimension for innovations in computerized testing. Parshall et al. (2002) introduced five innovation dimensions of CBTs: item format, response action, media inclusion, level of interactivity, and scoring method. Each of the five innovation dimensions will be discussed below.

\section{Dimensions of Innovation}

\section{Item Format}

The first dimension is the item format, and this dimension makes reference to the response possibilities of the test taker. The multiple-choice item format probably is the most well-known item type, and can also be used in paper-and-pencil tests. Multiple-choice items fall into the category of so-called selected response formats. The characterizing feature of these formats is that the test taker is required to select one or multiple answers from a list of alternatives. In contrast, constructed response formats require test takers to formulate their own answers, rather than select an answer from a list of alternatives (Drasgow \& Mattern, 2006). A fill-in-the-blank item type is an example of constructed response format, but essay questions and short answers are also constructed response items. All of the selected- and constructed-response item types can be administered by computer and, even more importantly, a growing amount of innovative item types are uniquely being designed for CBTs. 
Scalise and Gifford (2006) present a categorization or taxonomy of innovative item types for technology platforms. The researchers have identified seven different item formats, and 28 corresponding item examples (four per category) after a profound literature search, and reported these item examples in their paper. Most of the 28 item types are deliverable via a PC; however, some item types have specific advantages when computerized. For example, categorization, matching, ranking and sequencing, and hot-spot items are item types that are most efficiently administered by computer, compared to paper-and-pencil administration. Innovations in item format demonstrate that innovation is actually twofold. On the one hand, we can create new item types to measure constructs differently (improved measurement). On the other hand, we can also create new item types to measure completely different constructs that were difficult to measure before. This will also hold for the other dimensions of innovation, as will become clear in the following sections.

\section{Response Action}

The second innovation dimension is response action, and this dimension represents the physical action(s) a test taker has to perform in order to answer a question. The most common response action is of course filling in an answer sheet of a multiple-choice test in a paper-and-pencil test, or mouse clicking in a CBT. However, computerized testing software and computer hardware offer some interesting features for response actions. For example, test takers can also report their answers by typing on the keyboard, or speak them into a microphone (possibly integrated with voice recognition software). These types of response actions can hardly be called innovative nowadays, because they have been available for quite some time now. However, they show the constant progress in educational testing, influenced by the technological revolution.

Response actions in CBTs of skill assessment have been studied for the last two decades, with researchers looking for possibilities to assess skill in a way such that the response action corresponds with the actual skill under investigation. For example, joysticks, light pens, touch screens, and trackballs were used by the test takers as tools for the response actions. This resulted in another stream of innovations in assessment. The current innovations in assessment show that a whole new movement of response actions is emerging. 
Researchers are trying to unite response action and skill assessment, for example, through virtual environments, serious gaming, camera movement recognition, simulation software, and other innovative technologies that require test takers to physically perform a range of actions (e.g., a flight simulator). Van Gelooven and Veldkamp (2006) developed a virtual reality assessment for road inspectors. Because traffic density keeps on rising, road inspectors have taken over some tasks that used to be the duty of the traffic police, for instance, signaling to drivers, towing cars, and helping to fill in insurance documents after accidents. The test takers (road inspectors) are confronted with a virtual reality projected on a white screen. The director starts a specific case, and test takers can walk through the virtual environment with a joystick. During the assessment, all sorts of situations or problems develop, and the test takers are required to carry out actions with their joystick in the virtual environment. This example shows how assessments can be designed with innovative use of the response actions (controlling a joystick) a test taker has to perform.

Future innovations in CBT will repeatedly use more of these types of response actions, all the more because they unveil the possibility of measuring constructs that were difficult to measure before, or to measure constructs more accurately than we (as assessment experts) were able to in the past.

\section{Media Inclusion}

The third dimension is media inclusion, and indicates to what extent innovative CBTs incorporate (multi)media elements. Addition of media elements to CBTs can enhance the tests' coverage of the content area and may require test takers to use specific (cognitive) skills. Moreover, another key advantage of media inclusion is improved validity. Yet another advantage is that reading skills cannot be considered a confounding variable anymore. Media that are regularly found in CBTs are, among others, video, graphics, sound, and animations. The simplest form is providing a picture with an item stem, as is sometimes the case in paper-and-pencil tests. Ackerman, Evans, Park, Tamassia, and Turner (1999) have developed such a test of dermatological disorders that provides test takers with a picture of the skin disorder. Following presentation of the picture, the test taker is asked to select the disorder from a list on the right side of his screen. 
The assessment remains rather "static"; however, it would be a more complex assessment form if test takers had to manipulate the picture provided with the item, for example, by turning it around or fitting it into another picture. Still more difficult are items in which test takers have to assemble a whole structure with provided figures or icons, for example, when they have to construct a model and the variables are provided.

Audio is most often used in foreign language tests, and usually requires test takers to put on headphones. However, other fields have also used audio in (computerized) testing. For example, the assessment of car mechanics sometimes relies upon sound. Test takers have to listen to recorded car engines and indicate which cars have engine problems. In addition, medical personnel are presented with stethoscope sounds during assessment, and they are asked which sounds are unusual. Another innovative application of sound in assessment is to present questions in sound for people who are dyslexic or visually-impaired.

Video and animations are other media elements that may be incorporated into CBTs. These media elements are highly dynamic, and are highly congruent with authentic situations that test takers will face outside of the assessment situation. Several researchers have carried out case studies in which assessment included video. Schoech (2001) presents a video-based assessment of child protection supervisor skills. His assessment is innovative because it incorporates video in the assessment, but it is not highly interactive. The test takers watch a video, and then answer (multiple-choice) questions about the video that they have just watched. Drasgow, OlsonBuchanan, and Moberg (1999) present a case study of the development of an interactive video assessment (IVA) of conflict resolution skills. Because they introduce an innovative idea for making a CBT relatively interactive, their study is described below, in the section about the level of interactivity (the fourth innovation dimension) of a CBT.

\section{Level of Interactivity}

Interactivity, the fourth dimension of innovation, indicates the amount of interaction between test taker and test. As such, paper-and-pencil tests have no interaction at all. All test takers are presented with the same set of items, and those do not change during the administration of the test. In contrast, CBTs may also be highly interactive because of an adaptive element. Computerized adaptive tests (CATs) compute which item should be presented to a test taker based upon the answers given to all previous items. 
In that way, the CAT is tailored to the proficiency level of the test taker (Eggen, 2008, 2011). CATs are now widely used in assessment (both psychological and educational), but were initially a huge innovation made possible by the explosive growth of PCs and technology, and the introduction of Item Response Theory (IRT).

Another form of interactivity, also based on the concept of adaptive testing, is the incorporation of a two- or multistep branching function, possibly accompanied by video. Drasgow et al. (1999) present such a case study of an innovative form of a CBT. The CBT is structured upon two or more branches, and the answer(s) of the test taker form the route that is followed through the branches. The IVA of conflict resolution skills presented by Drasgow et al. required test takers to first watch a video of work conflict. Test takers then had to answer a multiple-choice question about the video. Following their answers, and depending upon their answers, a second video was started, and the cycle was completed once more. In essence, the more branches you create, the higher the assessment scores on interactivity, because it is highly unlikely that two test takers will follow exactly the same path.

Developing assessments that score high on the interactivity dimension is rather difficult, especially compared to some of the other innovation dimensions. Test developers are required to develop enough content to fill the branches in the adaptive interactive assessment. Another difficulty is the scoring of interactive CBTs. As test takers proceed along the branches of the interactive assessment, it becomes more difficult to use objective scoring rules, because many factors play a role, including weighing the various components of the assessment, and the dependency among the responses of the test taker. However, innovation in the level of interactivity has the potential to open up a wide spectrum of previously immeasurable constructs that now become available for measurement.

\section{Scoring Method}

The fifth and final innovation dimension is the scoring method. High-speed scanners were one of the first innovations in automatic scoring of paper-and-pencil multiple-choice tests. Automatic scoring possibilities have been developing rapidly, especially in the last two decades. Innovative items that score relatively low on interactivity and produce a dichotomous score are not too difficult to subject to automatic scoring. 
Other innovative CBTs, for example, complex performance-based CBTs, may require scoring on multiple dimensions, and are much more difficult to subject to automatic scoring. In performance assessment, the process that leads to the product is sometimes equal to or even more important than the product itself; however, it is a complicated task to design an automatic scoring procedure for process responses as well as product responses in complex performance-based CBTs.

Consider, for example, the above-mentioned branching of CBTs that incorporate video as well. Response dependency can be an obstructive factor for the scoring of these types of CBTs. This means that test takers' responses on previous items may release hints or clues for subsequent items. An incorrect answer on an item, after a test taker has seen the first video in the IAV, releases another video that may give the test taker a hint to his mistake on the previous item. Another issue is the weighing of items in a multistep CBT. Do test takers score equal points for all items, or do they score fewer points for easier items that manifest themselves after a few incorrect answers by the test taker?

Automated scoring systems also demonstrate some key advantages for the grading process of test takers' responses. The number of graders can be reduced, or graders can be completely removed from the grading process, which will also eliminate interrater disagreement in grading. Researchers have found that automated scoring systems produced scores that were not significantly different from the scores provided by human graders. Moreover, performance assessment of complex tasks is especially costly; molding these assessments into a CBT is extremely cost and time efficient. Thus, computers offer many innovative possibilities for scoring test takers' responses. For example, the use of text mining in assessment or classification is possible because of innovations in computer-based scoring methods. Text mining refers to extracting interesting and useful patterns or knowledge from text documents. This technique provides a solution to classification errors, because it reduces the effects of irregularities and ambiguities in text documents (He, Veldkamp, \& Westerhof, this volume). Yet another stream of innovation in scoring lies in test takers' behavior, and results in the scoring or logging of mouse movements, response times, speed-accuracy relationships, or eye-tracking. The key point that flows forth from the five innovation dimensions described above is twofold: not only do test developers become more capable of measuring constructs better, they also find themselves in a position to measure new constructs that were difficult to measure before. 


\section{Opportunities of Innovations in CBT}

\section{Performance Assessment and CBTs}

One of the main objections to multiple-choice tests is that, although extremely accurate in measuring declarative knowledge, they are difficult to design to measure test takers' skills and abilities. Thus, integrating performance assessment with CBT is an interesting opportunity for innovative CBTs. Several research and educational institutions have started research programs to explore the possibilities of measuring test takers' skills in complex performance-based tasks. Clyman, Melnick, and Clauser (1999) found that the correlation between computer-based case simulations and declarative knowledge assessment was only 0.5. Traditional tests and interactive case simulations presented via the PC therefore measure different domains of knowledge, and even make it possible to measure completely different constructs. The challenge lies in using a PC's capability to provide assessment exercises that revolve around the core of the actual performance of a task. In that way, the link between CBT and performance assessment becomes stronger, and the construct under investigation corresponds in both assessment types. In other words, both assessments are measuring the same construct, although in different ways.

\section{Media in CBTs}

Another opportunity in innovative CBTs is the inclusion of media within the CBT. Above, media inclusion is briefly discussed as one of the five innovation dimensions. Media inclusion in itself does not make a CBT innovative. However - and this is really the starting point of adding media into a CBT-being innovative should improve measurement. As mentioned above, it should either enhance the measurement of constructs that are now measured by other assessment types, or it should enable us to measure constructs that we were previously unable to measure.

Consider, for example, the CBT designed by Ackermann et al. (1999), which is briefly described in the section about media inclusion, as one of the five innovation dimensions. The addition of pictures of dermatological disorders in their multiple-choice test made it possible to more effectively measure test takers' knowledge about the skin disorders under investigation. This example represents how media inclusion can enhance or improve the measurement of constructs that were previously measured only by a multiple-choice test. In this case, it is the test takers' ability to recognize and identify different types of skin disorders that can be more accurately measured, but the possibilities that media offers are almost infinite. 


\section{Novel Item Types in CBTs}

A third opportunity that CBTs offer is the modification of item types, and the creation of novel item types. Again, the central idea is that they should improve the quality of measurement, or introduce new measurement possibilities. Paper-and-pencil tests impose restrictions on item construction. For example, it is almost impossible to administer drag-and-drop questions in a paper-and-pencil test, while computers offer great opportunities to integrate these questions within a CBT. Zenisky and Sireci (2002) describe 21 new types of item formats in their paper. Describing all item types is beyond the extent of this chapter; however, important to note here is that innumerable innovative item types have emerged in the literature on CBT item types in recent decades. Also, adaptations of these item types already exist, which shows that innovations and opportunities are almost countless. Computers offer opportunities such that new item types make it possible to access measurement to constructs that were very difficult or cost- and timeconsuming to measure in the past.

\section{Scoring of CBTs}

Improved scoring is a fourth and final opportunity that flows forth from computerized tests. The emphasis in this section will be on automated scoring of CBTs that aims to measure performancebased constructs or score performance-based tasks. Typically, these types of assessments require human graders, and sometimes actors or even walk-ons. Cost and time constraints are some serious disadvantages that go hand in hand with performance assessment. Automated scoring systems are being developed, and some are already operational, that make it possible to remove human graders from the scoring process, or at least minimize human intervention in the scoring process. One opportunity is, of course, to design automated scoring systems that make it possible to score test takers' attributes or behaviors in a way that we were not able to do before. Another opportunity is that automated scoring systems are more consistent and are able to extract more information than human graders (for example, text mining: He and Veldkamp (2012).

Moreover, Williamson, Bejar, and Hone (1997, cited in Dodd \& Fitzpatrick, 2002) found that human graders mostly let the automated score stand when provided with the analyses that resulted from the automated scoring. 
The automated scoring of essays, for example, becomes more and more incorporated as a regular and common method for scoring essays. Burstein et al. (1998) found that there was an $87 \%$ to 94\% agreement between the scores on essays that resulted from automated scoring systems and the scores awarded by the human graders. Above that, and equally important, this is not different from having two human graders score an essay independently. These findings support the idea that automated scoring systems are also applicable in the scoring of rather complex performancebased tasks. One concern that results from applying automated scoring systems in performancebased assessments is that it gives test takers the opportunity to actually lure the system into assigning a higher grade, for example, by using specific words that are scored by the system, but not properly used in the context of the essay. This is one risk that originates from the utilization of automated scoring systems, and innovations in CBTs in general. In the following section, other risks that might affect CBTs are discussed.

\section{Risks of Innovations in CBT}

CBTs offer a lot of opportunities, and the educational field can greatly benefit from these opportunities. However, every medal has two sides, and the other side of CBTs is that they are also subjected to several (potential) risks. For example, CBTs can be very costly and difficult to develop. Moreover, it may take a substantial amount of time to validate a CBT and, even if validated, questions about the efficiency of the CBT still remain. Finally, another threat to proper functioning of a CBT is test security and test disclosure.

\section{Development}

Guidelines on the development of classical multiple-choice tests are widely available in innumerable textbooks and publications. In contrast, such guidelines on the development of innovative CBTs did not exist up till now. Although many different guidelines exist that regulate the development of multiple-choice tests, it still takes a lot of time to develop a valid and reliable multiple-choice test. Consider the development of an innovative CBT without a frame of reference or any strong guidelines on these types of CBTs. It should now be possible to imagine that the development of a valid and reliable innovative CBT will require a lot of creativity, educational assessment expertise, and hard work. Such a process may endure multiple years and, by the time the assessment is completely developed, it may be outdated. 
Or as Schoech (2001) subtly notes: Walk the fine line between current limitations and potentials. Exam development is often a multi-year process, yet technology changes rapidly in several years. Thus, a technology-based exam has the potential to look outdated when it is initially completed.

\section{Validity of Scoring}

Another risk imposed on innovations in CBTs is validity. Most concerns raised about the validity of CBTs are related to the scoring procedure. If test developers create CBTs that automatically score constructed responses (e.g., scoring of essays by a computer), they have to be cautious about some pitfalls. For example, Powers et al. (2002) invited several parties in their study to "challenge" (i.e., try to trick) e-rater, which is an automated essay scorer. Participants' essays were also graded by humans, and the difference between the two types of scoring served as a dependent variable. The researchers found that the challengers were more successful in tricking the $e$-rater to award them higher scores than they deserved, based upon the human graders, than tricking the e-rater to award them lower scores than they deserved based upon the human graders. This short example stresses the importance of further research on automated scoring of (complex) tasks in CBTs.

\section{Test Security}

One of the most significant risks that stems from CBTs is test security. First of all, the ICT structure needs to be sufficient, thereby making it impossible for potential cheaters to hack a computer or network to get access to the questions in an item bank. Also, Internet-based CBTs make it possible to have test takers take tests at their own convenience, independent of time and place. This may expose the test to other test takers, and thereby impose a serious threat to test security. Another possible threat to the security of a test is test or item disclosure. Disclosure of a whole test or even several items may result in higher scores for subsequent test takers (Drasgow, 2002).

Finally, remaining in the same item bank for extended periods of time may result in diminished security of the test, because test takers are able to write down items, and may distribute them via the Internet, for example. 


\section{Future Research}

The focus of future research in CBT will be on improving the measurement of skills and performance abilities. Computers enable test developers to create high-fidelity computer simulations that incorporate innovations on all of the dimensions discussed above. Those types of CBTs are designed with the goal of measuring skill and demonstrating performance. Additionally, they correspond to actual task performance to a great extent, which is defined as the authenticity of an assessment. Therefore, these CBTs rely more upon the concept of authenticity than multiple-choice tests do, for example. Integration of multimedia, constructed response item types, highly interactive designs, and new (automatic) scoring methods will lead to an assessment form that closely approximates performance assessment in its physical form. Future research should determine to what extent it is possible to have computers adopt tasks that were usually part of performance assessment.

Furthermore, the psychometric performance of innovative CBTs that integrate many of the innovation dimensions should be determined. For example, studies on the validity of CBTs that are based on the innovation dimensions are all but absent in the current literature. Other studies should try to make innovative CBTs equivalent with performance assessment. Reliability has always been an important issue in performance assessment, which usually relies upon human graders. Innovative CBTs may be able to integrate high reliability with authentic assessment forms. Therefore, researchers should also focus on the concept of reliability in innovative CBTs. Automated scoring systems that rely upon IRT algorithms, or the integration of computerized adaptive testing (CAT), are other interesting future research directions within CBT.

The research on innovations in CBTs that has been reported in the scientific literature mainly focuses on cognitive performance tasks, usually in higher education. Some case studies exist that have tried to measure particular constructs in skill-based professions, for example, in the medical professions or ICT. However, future research should also focus on measuring skill constructs in vocational professions that rely upon physical skills rather than cognitive or intellectual skills.

The continuing technological revolution makes it possible for test developers to further innovate, create, and revolutionize CBTs. The coming decade will be very interesting for the educational measurement field, and there is a whole new range of CBTs to look forward to. 


\section{References}

Ackerman, T.A., Evans, J., Park, K.S., Tamassia, C., \& Turner, R. (1999). Computer assessment using visual stimuli: A test of dermatological skin disorders. In F. Drasgow \& J.B. OlsonBuchanan (Eds.), Innovations in computerized assessment (pp. 137-150). Mahwah, NJ: Erlbaum.

Burstein, J., Braden-Harder, L., Chodrow, M., Hua, S., Kaplan, B., Kukich, K., et al. (1998). Computer analysis of essay content for automated score prediction (ETS Research Report RR-98-15). Princeton, NJ: Educational Testing Service.

Clyman, S.G., Melnick, D.E., \& Clauser, B.E. (1999). Computer-based case simulations from medicine: Assessing skills in patient management. In A. Tekian, C.H. McGuire, \& W.C. McGahie (Eds.), Innovative simulations for assessing professional competence (pp. 2941). Chicago, IL: University of Illinois, Department of Medical Education.

Dodd, B.G., \& Fitzpatrick, S.J. (2002). Alternatives for scoring CBTs. In C.N. Mills, M.T. Potenza,

Drasgow, F. (2002). The work ahead: A psychometric infrastructure for computerized adaptive tests. In C.N. Mills, M.T. Potenza, J.J. Fremer, \& W.C. Ward (Eds.), Computer-based testing: Building the foundation for future assessments (pp. 1-35). Mahwah, NJ: Erlbaum.

Drasgow, F., \& Mattern, K. (2006). New tests and new items: Opportunities and issues. In D. Bartram \& R.K. Hambleton (Eds.), Computer-based testing and the internet: Issues and Advances (pp. 59-75). Chichester: Wiley.

Drasgow, F., Olson-Buchanan, J.B., \& Moberg, P.J. (1999). Development of an interactive videoassessment: Trials and tribulations. In F. Drasgow \& J.B. Olson-Buchanan (Eds.), Innovations in computerized assessment (pp. 177-196). Mahwah, NJ: Erlbaum.

Eggen, T.J.H.M. (2008). Adaptive testing and item banking. In J. Hartig, E. Klieme, \& D. Leutner (Eds.), Assessment of competencies in educational contexts (pp. 215-234). Götting en: Hogrefe.

Eggen, T.J.H.M. (2011, October). What is the purpose of CAT? Presidential address at the 2011 Meeting of the International Association for Computerized Adaptive Testing, Monterey, CA.

Fremer, J.J. \& W.C. Ward (Eds.), Computer-based testing: Building the foundation for future assessments (pp. 215-236). Mahwah, NJ: Erlbaum. 
Gelooven, D. van, \& Veldkamp, B. (2006). Beroepsbekwaamheid van weginspecteurs: een virtual reality toets. In E. Roelofs \& G. Straetmans (Eds.), Assessment in actie:Competentiebeoordeling in opleiding en beroep (pp. 93-122). Arnhem, the Netherlands: Cito.

He, Q \& Veldkamp, B.P.(2012). Classifying unstructured textual data using the Product Score Model: an alternative text mining algorithm. In T.J.H.M. Eggen \& B.P. Veldkamp (Eds.), Psychometrics in Practice at RCEC: (pp. 47-63). Enschede, Netherlands: RCEC.

Parshall, C.G., Spray, J.A., Kalohn, J.C., \& Davey, T. (2002). Practical considerations in computer-based testing. New York, NY: Springer.

Powers, D.E., Burnstein, J.C., Chodorow, M., Fowles, M.E., \& Kukich, K. (2002). Stumping $e$ rater: Challenging the validity of automated essay scoring. Computers in Human Behavior, 18, 103-134.

Scalise, K., \& Gifford, B. (2006). Computer-based assessment in e-learning: A framework for constructing "intermediate constraint" questions and tasks for technology platforms. Journal of Technology, Learning, and Assessment, 4(6). Retrieved [March 20, 2012] from http://www.jtla.org.

Schoech, D. (2001). Using video clips as test questions: The development and use of a multimedia exam. Journal of Technology in Human Services, 18(3-4), 117-131.

Zenisky, A.L., \& Sireci, S.G. (2002). Technological innovations in large-scale assessment. Applied Measurement in Education, 15, 337-362. 


\title{
Chapter 13
}

\section{Towards an Integrative Formative Approach of Data-Driven Decision Making, Assessment for Learning, and Diagnostic Testing}

\section{Jorine A. Vermeulen and Fabienne M. van der Kleij}

\begin{abstract}
This study concerns the comparison of three approaches to assessment: Data-Driven Decision Making, Assessment for Learning, and Diagnostic Testing. Although the three approaches claim to be beneficial with regard to student learning, no clear study into the relationships and distinctions between these approaches exists to date. The goal of this study was to investigate the extent to which the three approaches can be shaped into an integrative formative approach towards assessment. The three approaches were compared on nine characteristics of assessment. The results suggest that although the approaches seem to be contradictory with respect to some characteristics, it is argued that they could complement each other despite these differences. The researchers discuss how the three approaches can be shaped into an integrative formative approach towards assessment.
\end{abstract}

Keywords: Formative Assessment, Data-Driven Decision Making, Assessment for Learning, Diagnostic Testing

\section{Introduction}

Within the various approaches to assessment, the importance of increasing student learning by acting on students' educational needs is emphasized. However, the meaning ascribed to student learning is one of the factors that separates these approaches to assessment: a distinction is made between learning outcomes and the process of learning. This means that some assessment approaches focus on what has to be learned, while other approaches focus on how students learn what has to be learned (best) and the quality of the learning process. Furthermore, assessment approaches differ in the aggregation levels in the educational system (e.g., student, classroom, school) at which the assessment is aimed. Due to these differences, the approach to assessment that is chosen affects the strategies that are used to assess and promote student learning outcomes. 
This chapter addresses the differences and similarities of the three approaches to assessment that are currently most frequently discussed in educational research literature. The first approach is Data-Driven Decision Making (DDDM), which originated in the United States of America as a direct consequence of the No Child Left Behind (NCLB) Act in which improving students' learning outcomes is defined in terms of results and attaining specified targets. Secondly, Assessment for Learning (AfL), originally introduced by scholars from the United Kingdom, is an approach to assessment that focuses on the quality of the learning process, rather than merely on students' (final) learning outcomes (Stobart, 2008). Finally, in Diagnostic Testing (DT), also referred to as diagnostic measurement, students' learning outcomes are described as students' learning processes and factors that resulted in students' success or failure to do particular tasks (Leighton \& Gierl, 2007a, 2007b; Rupp, Templin, \& Henson, 2010).

Although all three approaches claim to provide information that can be used to increase students' learning outcomes, there appears to be no clear study into the relations and distinctions between these approaches. More specifically, these terms are often used interchangeably. Interestingly, the literature on DDDM tends to cite literature concerning AfL, but not vice versa (e.g., Swan \& Mazur, 2011). The aim of this study is to investigate the extent to which DDDM, AfL, and DT can be integrated into an approach that can be used for maximizing student learning. By maximizing student learning we aim at both the process and outcomes of learning as optimized at all levels of education. The following research question will be answered: To what extent can DDDM, AfL, and DT be shaped into an integrative formative approach towards assessment?

\section{The Formative Function}

Tests are a crucial part of education, namely, it would not be possible to check whether a certain instructional activity led to the realization of the intended learning outcomes without testing (Wiliam, 2011). Paper-and-pencil tests are often used within the classroom to gather information about student learning. Besides paper-and-pencil tests, there are various other methods for measuring pupils' knowledge and abilities. For example, homework, projects, discussions, and observations can provide valuable information about student learning. Whenever such a broad spectrum of instruments is used for gathering information about student learning one speaks of assessment (Stobart, 2008). Traditionally, in education, a distinction is made between summative and formative assessment. 
Summative assessments can be used to judge or compare the learning outcomes of students, based on which a decision is made with regard to, for example, selection, classification, placement, or certification. There are also tests that have the purpose of directing the learning process.

These tests are called formative tests (Sanders, 2011). However, a test is not in itself formative or summative by definition (Stobart, 2008). Whether a test is used formatively does not depend on the characteristics of the test itself, but on the way the test results are being used, in other words, the function of the test results (Harlen \& James, 1997; Stobart, 2008). Whenever a test result plays a role in making (part of the) pass/fail decision, it fulfills a summative function. The same test, however, can also fulfill a formative function, for example, when feedback is provided to students that can be used in future learning. Another example of a formative use is a teacher using test results to evaluate the effectiveness of the instruction. Subsequently, the teacher might make amendments to the educational program in order to meet the needs of the learners.

Formative assessment is a broad concept that has many definitions (e.g., AfL, and diagnostic assessment; Bennett, 2011; Johnson \& Burdett, 2010). Initially, the formative concept was introduced by Scriven (1967) to indicate interim evaluation of intervention programs. In 1968, the formative concept was first used in the context of instruction by Bloom. In the years that followed, various meanings have been ascribed to formative assessment. Recently, researchers have come to the insight that a distinction between formative and summative assessment based on time-related characteristics is not a useful one. After all, it is the way that test results are eventually used that determines the purpose the test serves (Stobart, 2008).

Subsequently, for comparison of assessment approaches it is useful to distinguish between formative program evaluations and formative assessments (Shepard, 2005; Harlen, 2007). Formative program evaluations are meant to make decisions at a higher aggregation level than the level of the learner (e.g., classroom or a school) about the educational needs of pupils. Formative assessment, on the contrary, concerns decisions at the level of the learner. Results from formative assessments are used to accommodate the individual educational needs of pupils. In this study, we refer to formative evaluation as the evaluation of the quality of education. We will use the term formative assessment to indicate assessment that takes place within the classroom and is focused on improving instruction in the classroom and for individual pupils. In the following sections, we elaborate on the characteristics of the three views on assessment, after which the three approaches are compared. 


\section{Data-Driven Decision Making}

Teachers make most of their decisions based on their intuition and instincts (Slavin, 2002, 2003). However, educational policies such as NCLB have caused an increase in accountability requirements, which has stimulated the use of data for informing school practice in the United States of America. The main idea behind NCLB is that by setting standards and measurable goals the learning outcomes of pupils can be raised. Namely, research has pointed out that by setting specific learning goals an increase in student achievement can be obtained (Locke \& Latham, 2002). The idea of using data for informing instruction is not new, namely, in the 1980s there was an approach that attempted to make instruction more measurement-driven (Popham, Cruse, Rankin, Sandifer, \& Williams, 1985). Furthermore, recent studies draw attention to the importance of using data, such as assessment results and student surveys, in making decisions (Wayman, Cho, \& Johnston, 2007; Wohlstetter, Datnow, \& Park, 2008). When data about students are used to inform decisions in the school it is referred to as DDDM (Ledoux, Blok, Boogaard, \& Krüger, 2009).

Recently, DDDM has gained popularity in the Netherlands (the Dutch term that is often used is opbrengstgericht werken), which is seen as a promising method for increasing pupils' learning outcomes (Ledoux et al., 2009). Schildkamp and Kuiper (2010) have defined

DDDM as "systematically analyzing existing data sources within the school, applying outcomes of analyses to innovate teaching, curricula, and school performance, and, implementing (e.g., genuine improvement actions) and evaluating these innovations" (p. 482). The data sources that can be used to inform decisions are not only results from tests. Other usable data sources are school self-evaluations, characteristics of the pupils in the school, results from questionnaires taken by parents or pupils, and various assessments sources, such as an external national test or internal school assessments. In this study, for the sake of comparing the three approaches, we will focus on the use of student achievement results for informing the decision-making process. Data about student achievement will be referred to as data-feedback.

Many schools already possess data-feedback, for example from a student monitoring system. These data are often systematically collected via standardized tests and can therefore be valued as objective. Besides these objective data, teachers possess data-feedback from daily practice that has been gathered using various assessment methods. When data-feedback is used in the right way, meaning that its use will lead to education that is more adequately adapted to the needs of the learner, this will eventually lead to better learning results. 
At the student and classroom level, data-driven decision making can be a valuable instrument for using assessment results in a formative way. Assessment results are an important source of information about how learning processes could be improved for both students and teachers. Students need feedback to choose the most suitable learning strategies in order to achieve the intended learning outcomes, while teachers need data-feedback in order to act on the pupils' current points of struggle and to reflect on their own teaching practices. However, knowledge about how teachers use assessment results for instructional improvement is limited (Young \& Kim, 2010).

According to Wayman (2005), the NCLB policy carries the assumption that whenever data is available it will lead to changes in teaching practice. A general definition of educational measurement comprises four activities that are part of a cyclic process of evaluation: "...designing opportunities to gather evidence, collecting evidence, interpreting it, and acting on interpretations" (Bennett, 2011, p. 16). However, it is not always self-evident for practitioners how (accountability) data should be translated into information that can be readily used to make decisions in the school. Therefore, it is not surprising that recent studies suggest that data-feedback is underused in the majority of Dutch schools (Ledoux et al., 2009; Schildkamp \& Kuiper, 2010). These studies have found that the implementation of the evaluative cycle is incomplete in many schools. The results of these studies imply that students are frequently assessed and that the results of the assessments are registered, but that there is no subsequent use of the data-feedback. Moreover, research has also pointed out that many teachers indeed get stuck in the interpretation phase of the evaluative cycle (Meijer, Ledoux, \& Elshof, 2011). Thus, educators need to know how to translate raw assessment data into knowledge about student learning outcomes that indicates in what way students' learning outcomes can be optimized.

The literature makes a distinction between data and information (Davenport \& Prusak, 1998; Light, Wexler, \& Heinze, 2004; Mandinach, Honey, \& Light, 2006). Data are characterized as objective facts, which have no meaning. By interpreting data, they can be transformed into information, for example by summarizing, contextualizing and calculating (Davenport \& Prusak, 1998). Subsequently, information can be transformed into actionable knowledge by synthesizing and prioritizing. This actionable knowledge is the basis for a decision about which action to undertake (Light et al., 2004).

The impact of the action is evaluated by gathering new data, in this way a feedback loop is created (Mandinach et al., 2006). However, this is not an easy process because teachers are used to making decisions intuitively (Slavin, 2002, 2003). 
"As many educators say, they are data rich, but information poor. By this they mean that there is far too much information with which they must deal, but those data are not easily translatable into information and actionable knowledge" (Mandinach et al., 2006, p. 12).

Within the school, many feedback loops can exist. The frequency in which the feedback loops are completed depends, among other things, on the type of data-feedback that is used. Data-feedback from formal and objective tests, for example from a student monitoring system, are less frequently available than data from informal assessment situations, such as homework assignments. Ledoux et al. (2009) stated that the quality of the evaluative cycle is dependent upon the quality of the data-feedback. This implies that unreliable data can lead to making unjustified decisions. Moreover, wrongly interpreting data can also lead to making unjustified decisions. Being data-literate is thus a necessary prerequisite for successfully implementing DDDM.

\section{Assessment for Learning}

AfL focuses specifically on the quality of the process of learning instead of the outcomes of the learning process. Moreover, "it puts the focus on what is being learned and on the quality of classroom interactions and relationships" (Stobart, 2008, p. 145). The theory of AfL has no strict boundaries; it is part of a bigger entity in which curriculum, school culture, and instruction approaches intervene. It is noteworthy that AfL is viewed as a divarication of formative assessment (Johnson \& Burdett, 2010; Stobart, 2008).

The Assessment Reform Group (2002) defined AfL as follows: "Assessment for Learning is the process of seeking and interpreting evidence for use by learners and their teachers to decide where the learners are in their learning, where they need to go and how best to get there".

Klenowski (2009) reported on, what she named a 'second-generation definition' of AfL, which was generated at the Third Assessment for Learning Conference (2009): "AfL is part of everyday practice by students, teachers and peers that seeks, reflects upon and responds to information from dialogue, demonstration and observation in ways that enhance ongoing learning" (p. 264). This 'second-generation definition' was needed because definitions, or parts of them, are often misinterpreted (Johnson \& Burdett, 2010; Klenowski, 2009). 
AfL takes place in everyday practice, which means the process is characterized by dialogues between learners and the teacher. This also means that assessments are integrated into the learning process, which is contrary to the traditional approach where assessments are a separate activity that stands apart from instruction. Furthermore, Klenowski's (2009) definition emphasizes the role of the learners in the learning process and their autonomy. It also emphasizes the nature of AfL in terms of making decisions about which steps to take in the learning process. The information that is used to inform decisions can come from various assessment sources, such as dialogues and observations. These events can be both planned and unplanned. This implies that the evidence that is gathered about the learning process of the learners can be both qualitative and quantitative in nature. The last part of the definition stresses the formative function of assessments. One can only say an assessment serves a formative function when students and teachers use the information for informing and enhancing learning. Therefore, a crucial aspect of AfL is feedback, which is used to direct future learning (Stobart, 2008).The AfL approach encompasses more than the use of assessments and their results. The Assessment Reform Group (1999) described AfL using five core features:

1. Learners are actively engaged in their learning process;

2. effective feedback is provided to learners;

3. instructional activities are being adapted based on assessment results;

4. learners are able to perform self-assessment;

5. the influence of assessment on motivation and confidence of learners is acknowledged.

Stobart (2008) argued that AfL is a social activity that influences both learner identity and the type of learning that will take place.

Whenever the primary goal of testing is to measure a result this can lead to a misleading image of what the level of the learner is, because one cannot measure the full scope of a curriculum. Moreover, Stobart states that on some standardized tests results can improve without students actually learning more. Also, when too much emphasis is placed on achieving specific goals, by frequently using these types of standardized tests, the learning process is narrowed and teaching to the test is promoted (Stobart, 2008), which elicits surface learning (Harlen \& James, 1997). 
Therefore, multiple methods for gathering evidence about student learning will lead to a more complete picture of students' knowledge and abilities (Harlen \& Gardner, 2010), and are needed in order to achieve deep learning (Harlen \& James, 1997).

Hargreaves (2005) compared various definitions of AfL in the literature and interpreted definitions as formulated by teachers and head teachers in a survey. She concluded that there are two approaches within AfL; a measurement and an inquiry approach. In the measurement approach, AfL is viewed as an activity that includes marking, monitoring, and showing a level. In this view, (quantitative) data are used to formulate feedback and to inform decisions. Assessment is seen as a separate activity to show that a predetermined level has been achieved. On the contrary, in the inquiry approach, AfL is a process of discovering, reflecting, understanding and reviewing. It is very much focused on the process and assessments are integrated into the learning process. Qualitative sources of information play an important role. In both approaches, feedback is used to steer future learning. However, in the first approach, feedback might be less immediate and less suited to meet the needs of the learners because of the more formal character of the assessments.

In our opinion, the measurement approach towards AfL can easily turn into a misinterpretation. To illustrate our point of view we will take the example of monitoring (personal communication, M. Johnson, January 3, 2012). Monitoring in itself does not have to be bad in the light of AfL. Teachers can keep accurate track of students' learning progress and this can help them to make more informed decisions about their students. This way, quantitative data can be used to inform qualitative actions, such as providing elaborated feedback. However, as soon as the monitoring takes place at a level higher than the class level, the measures used to monitor student learning lack a contextual link to the particular performances to which they relate. In other words, the distance between the monitoring action and the learner is too large, which makes it lose its qualitative potential. At this level, monitoring is reduced to ticking boxes, which is at odds with the spirit of AfL (Johnson \& Burdett, 2010).

\section{Diagnostic Testing}

Making diagnoses originates from the field of physical and mental health care in which the aim is to diagnose a disease or disorder and to advise on the treatment (De Bruyn, Ruijssenaars, Pameijer, \& Van Aarle, 2003; Kievit, Tak \& Bosch, 2002). 
In education, DT was initially used for identifying students who were unable to participate in mainstream education because of their special educational needs. Currently, DT is still used for the identification of students with learning deficiencies and/or behavioral problems. However, it is currently also believed that for instruction to be effective educators need to take into account all students' learning needs (Wiliam, 2011).

Before addressing the process of diagnosing the educational needs of students, the distinction between formative assessment and DT will be explained, as well as the difference between DT and diagnostic assessment. Articles on formative assessment sometimes use the concept of diagnosing when explaining the purpose of formative assessment (e.g., Black \& Wiliam, 1998). Similarly, in the literature on DT (or assessment), DT has been defined as equal to formative assessment (e.g., Turner, VanderHeide, \& Fynewever, 2011). However, not every diagnostic test fulfills a formative function because, as we explained in the sections above, whether or not a test serves a formative purpose depends on how the results of that test are used. According to Keeley and Tobey (2011), DT is mainly concerned with measuring students' preconceptions and reasoning styles. This includes the identification of the use of inadequate reasoning styles, and skipped or wrongly executed procedural steps as a result of, among other things, misconceptions. Formative tests, on the other hand, take into account any information about students' learning outcomes that can be used for adapting instruction and providing feedback. In our view, a diagnostic test fulfills a formative function when the diagnosis is used to optimize students' learning processes.

Moreover, we make a distinction between DT and diagnostic assessment. DT refers to the use of computerized (adaptive) diagnostic tests, whereas diagnostic assessment refers to the use of various assessment methods, such as diagnostic interviews (Moyer \& Milewicz, 2002). Diagnostic assessment is very time-consuming and labor-intensive, and mainly uses qualitative methods. On the other hand, DT is less time-consuming and labor-intensive for teachers, because students can work independently and the test results are delivered automatically in a format that can readily be used to support decision making.

From a diagnostic testing point of view, the development of diagnostic tests requires (statistical) measurement models that fulfill the role of the diagnostician. It also means that data gathered with these tests are likely to be more objective and reliable than alternative assessment methods. 
And also, even though data obtained with a diagnostic test have a quantitative nature, the reportage of that test can be the qualitative interpretation of those data. It goes beyond the scope of this chapter to elaborate on these measurement models, therefore we refer the interested reader to Leighton and Gierl (2007b) and Rupp, Templin, and Henson (2010).

The utility of DT stems from its potential to inform instructional decisions by providing information about students' learning needs. Stobart (2008, p. 55) described diagnosing learning needs as: "...[identifying] how much progress can be made with adult help...". This statement is in accordance with Vygotsky's criticism on the test culture of the 1930s.

He believed that in order to promote student learning, tests should focus on what students are able to learn, rather than what they have learned so far (Verhofstadt-Denève, Van Geert, \& Vyt, 2003). In other words, tests should assess a student's zone of proximal development (ZPD); which is defined as what a student can do with the minimal help of adults or peers (Verhofstadt-Denève et al.).

There are multiple ways of assessing the ZPD, for example, a diagnostic test may include tasks in which the student is offered help in terms of feedback when an incorrect answer is given (e.g., a number line in an arithmetic test). This kind of help is also known as scaffolding (Verhofstadt-Denève et al., 2003). Note that a diagnostic test will become more similar to what can be described as learning material when students receive rich feedback about their mistakes.

Another method to assess the ZPD is to diagnose students' line of reasoning when solving the tasks within the diagnostic test. From a cognitive psychological point of view, student ability is more than being able to solve specific tasks; how students derive the answers to the items is viewed as an important indicator of students' ability levels. For this method of DT a cognitive diagnostic model is necessary, meaning a theory about how students with different ability levels solve the tasks that are the object of assessment (Leighton \& Gierl, 2007a, 2007b). Such a theory includes commonly developed misconceptions and errors that are frequently observed, and their relation to different ability levels. Because students within similar cultural and educational contexts are likely to follow comparable learning processes, it is more efficient to focus on frequently observed errors than on identifying students' errors that are uncommon or the result of 'slips' (Bennett, 2011). Additionally, Bennett explained that the identification of 'slips' has less diagnostic value because they cannot inform teachers about how to change their practice. 
Whatever method for developing a diagnostic test is used, it is the process of developing "...items (i.e., tasks, problems) that can be used to efficiently elicit student conceptions that these conceptions can be related back to a hypothesized learning progression" (Briggs \& Alonzo, 2009, p. 1). A similar cyclic process of formulating and testing hypotheses about the nature of students' achievements is followed within child health care services. Although variations might exist, the diagnostic cycle consists of the following four phases (De Bruyn et al., 2003; Kievit et al., 2002):

1. Identifying the problem (complaint analysis);

2. clarifying the problem (problem analysis);

3. explaining the problem (diagnosing); and

4. indication of treatment possibilities (advising and deciding).

An example of diagnosing mathematics difficulties following phases similar to the four phases of the diagnostic cycle is described by Rupp et al. (2010, p. 14).

Table 1 illustrates how each phase of the diagnostic cycle could be used in educational contexts. The first three phases result in rich descriptions of the student's knowledge and skills, whereas the fourth phase will result in a diagnosis that prescribes which decision concerning the learning environment will have the highest probability of successful outcomes for the student. As explained in the second paragraph of this section, a diagnostic test can only have a formative function when it is used to improve students' learning processes. As shown in Table 1, this is the case when Phase 4 is completed. Furthermore, we consider DT an approach to assessment that primarily focuses on the learning processes of individual students. For that reason, we frequently refer to the student. However, from a practical point of view, it might be more feasible for teachers to address the needs of (small) groups of students. This could be done by classifying students into, for example, instruction groups based on the similarity of their diagnosis. However, caution is needed with this approach because (depending on the measurement model used) DT is not meant for comparing students. Also, because of the degree of detailed information, it might be difficult to group students with a similar diagnosis who would benefit from the same adaptations to the learning environment. 
Table 1 Objectives and Outcomes of the Four Phases of the Diagnostic Cycle

Diagnosis Type Objective Outcome(s)

Descriptive

Acknowledging Assessing whether the student's learning

The probability of the student reaching process is optimized given the student's

(Phase 1) characteristics and the characteristics of the learning environment.

With this diagnosis it can only be decided whether a student might benefit from adaptations to the learning environment.

Exploratory

Describing student's current achievement on the test in terms of

(Phase 2) strengths and weaknesses.

Explanatory

(Phase 3)
Investigating which hypotheses about the nature of the student's achievements on the test are most probable. learning goals given his/her current test performance on tasks that are associated with knowledge and abilities necessary for achieving those goals.

A list of strengths (i.e., things he can do or knows that are improbable based on his overall performance on other domains) and a list of weaknesses (i.e., things he cannot do or does not know that are improbable based on his overall performance on other domains).

A description concerning which errors the student has made and why these errors occurred. The student report might describe the relation between the student's errors, misconceptions, as well as his wrongly applied or skipped procedural steps.

\section{Prescriptive}

(Phase 4)

a. Indication
b. Selection
c. Classification

a. Determining which intervention or changes to the learning environment are most likely to be effective in optimizing the student's learning process.

b. Determining whether the student should be selected.

c. Determining to which group the student belongs.
Advice about the actions that are most likely to result in optimization of the student's learning process.

\section{Comparing the Three Approaches to Assessment}

Rupp, Templin, and Henson (2010, p. 12) organized various uses of diagnostic assessment into seven categories:

1. The object of the assessment;

2. the object of the decision;

3. the time point in which decisions need to be made; 
4. the objective of the assessment;

5. the assessment methods;

6. the types of intervention; and

7. the power differentials between agents.

In order to compare DDDM, AfL, and DT we applied these characteristics of assessment to all three approaches. This method enables us to systematically identify differences and similarities between the approaches. Additionally, we added 'characteristics of the assessment process' and 'learning theory'. Also, we broke down some of the features to smaller aspects. The explanation of the assessment features and their aspects are described in Table 2.

Table 2 Characteristics of Assessment Used to Compare the Three Assessment Approaches

\section{Assessment Characteristic}

1. The object of the assessment.

2. The object of the decision.

3. The time point in which decisions need to be made.
a. Timing of the decision
b. Frequency of the decision
c. Timing of the feedback

4. The objective of the assessment.

5. The assessment method.

6. Characteristics of the assessment process.

7. The power differentials between agents.

a. Who makes the decision.

b. Who has to take actions (e.g., providing feedback, deciding what has to be learned).

8. The types of intervention.

9. (Learning) theory.

\section{Explanation}

Level at which the data is collected (i.e., individual student(s) or groups of students).

Level at which the decision is aimed, which should be equal to the level at which data are aggregated.

The timing of assessment, decision and actions that follow the decision.

- Who should benefit from the decision?

- What behavioral and cognitive aspects are most important?

- Degree of standardization.

- The resulting data (qualitative vs. quantitative).

- Cyclic vs. non-cyclic.

- Systematic vs. non-systematic.

Discrepancies between the agents who make the decision and the agents who are expected to follow-up on the decisions by taking action. The higher the differential, the higher the stakes.

The type of intervention that follows assessment. Formative interventions can be:
a. proactive;
b. retroactive; or
c. interactive (Stobart, 2008, pp. 146-147).

On which learning theories the assessment approach is based. 
First, we investigated how the object of assessment (e.g., students, teachers, or schools) as well as the object of the decision making varies across assessment approaches. The reportage of the results should equal the level at which the decision is aimed because it is, for example, more difficult to base decisions about students on data reported at classroom level. Next, we compared the three approaches on the timing of the assessment, which is affected by the time in which the decision has to be made. With this third feature the frequency of assessment was investigated as well. The timing of feedback is included to compare the approaches on the timing of actions taken after the decision.

The fourth assessment characteristic is the objective of assessment, by which it was analyzed who should benefit from the decision, as well as which behavioral and cognitive aspects are essential to complete the assessment task. The behavioral and cognitive aspects that are the primary objective of the assessment affect the assessment method that is most likely to be used. We compared the assessment methods used in each approach on the degree of standardization and the type of data that are collected. Sixth, the assessment process consists of collecting data, interpreting data, making a decision, and taking actions. The assessment approaches differ in the extent to which the assessment process is cyclic and systematic. When the assessment process is very systematic and follows a strict cycle the procedure becomes more formal, whereas a non-systematic and non-cyclic assessment process will be perceived as informal and more integral to the learning process. This degree of formality, in combination with the degree of standardization of the assessment method, affects how students will perceive the stakes of the assessment.

Another assessment feature that affects the stakes of assessment is the power differentials between agents. Power differentials might exist between the object of assessment, the assessor, the decision maker, and the person who follows through with the decision by implementing the intervention. The eighth characteristic is the type of intervention that follows the decision. Because the aim of this comparison is to composite an integrative formative approach to assessment, we studied which formative interventions could follow each assessment approach. Assessment data can also be used for the sake of remediating learning difficulties, which is called retroactive formative assessment (Stobart, 2008). On the contrary, proactive formative assessment leads to the implementation of interventions that should prevent the development of learning difficulties by addressing commonly developed misconceptions. This type of formative assessment is also known as pre-emptive formative assessment (Carless, 2007). 
With interactive formative assessment the intervention is the result of the interaction of the learner with the learning environment (e.g., learning materials, teachers, and students), meaning that the line between assessment and intervention is blurred (Stobart, 2008).

Finally, a parallel was drawn between the approaches regarding the learning theories that are used to define what is meant by students' learning outcomes. Among these theories are behaviorism, (social) constructivism (which is based on the cultural historical theory of Vygotsky), cognitive developmental psychology of Piaget, and information processing theories (Verhofstadt-Denève et al., 2003).

\section{Results}

The results of the comparison between the assessment approaches are shown in Table 3. The numbers of the headings of the following sections correspond with the numbers of the characteristics of assessment in Table 3.

\section{The Object of the Assessment}

DDDM comprises all educational levels, which means that it considers both assessment and evaluation. AfL comprises classroom and individuals and DT concerns individuals. Since the three approaches comprise different levels at which data are gathered, this suggests they could complement each other.

\section{The Object of Decision Making}

The objects of the decisions differ across the three approaches. In DDDM, the decisions can concern all levels of education, whereas AfL only concerns decisions within the classroom. DT is merely aimed at decisions about individual students. The three approaches comprise different levels at which decisions are made, which suggests that these approaches could be present simultaneously. Figure 1 shows the objects of the decisions in the three approaches.

Figure 1 Objects of the Decisions in the Three Approaches

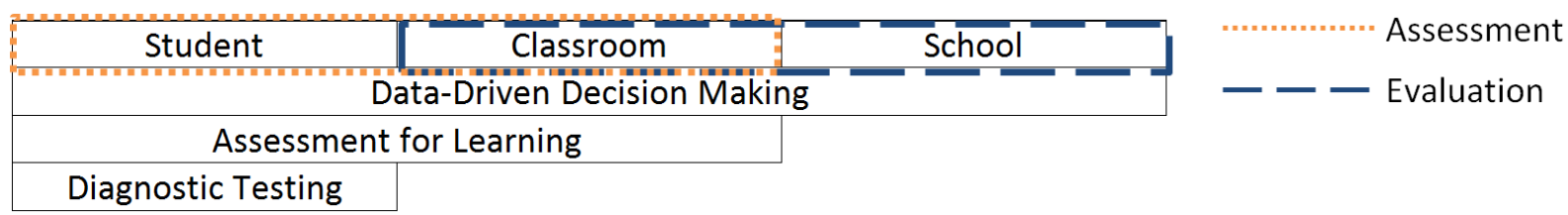




\section{The Time Point in Which Decisions Need to be Made}

\section{a. Timing of the decision}

In DDDM, the timing of the decision can vary from immediately to a couple of years after data collection. In AfL, the decisions are almost always made immediately during the learning process. In DT, the time between the measurement and decision depends on the needs of the learner(s), but it is desirable to keep this time limited.

\section{b. Frequency of the decision}

Depending on the stakes of the decision, in DDDM the frequency of the decisions varies from daily to once in a couple of years. High-stakes decisions require careful consideration, as well as reliable and objective data. These types of decisions are generally made less frequently compared to low-stakes decisions. In AfL, teachers continuously make decisions based on the assessment information at hand. Thus, decisions are made very frequently. For DT, the frequency of the decisions will depend on the needs of the learner(s).

c. Timing of the feedback

In DDDM, feedback is usually delivered with a delay; this is especially the case when feedback from a standardized measurement is delivered by an external party. In AfL, feedback is provided continuously according to the needs of the learners. When using DT, it is preferable to inform the learners about the diagnosis as soon as possible after the measurement.

\section{The Objective of the Assessment}

The objective of DDDM is assessing and/or evaluating whether or not learning goals are met, and thereby investigating whether changes to the learning environment are necessary. This approach is retroactive, because it aims to resolve problems after a period of teaching. On the other hand, the objective of AfL is improving the quality of the learning process and thereby establishing higher learner autonomy, and higher learning outcomes. This process is characterized as interactive and proactive, and sometimes also as retroactive (Stobart, 2008). The objective of assessment within DT is measuring the student's processing activities, and identifying their preconceptions, misconceptions, bugs, and problem solving strategies.

The process of DT is mostly retroactive, but it can also be used proactively. Furthermore, when the instrument used for DT provides feedback during the test, the process becomes interactive. 


\section{The Assessment Methods (Instruments)}

The assessment methods used in DDDM are primarily standardized tests that result in quantitatively reported data-feedback, which has to be interpreted by the user. Standardized, in this context, indicates that all students take the same test. Also, the design of the instrument is determined by the learning goals that are being assessed, meaning that emphasis is placed on what has to be learned.

Usually, highly reliable tests are used that can be scored automatically, so they are easy to administer on a large scale. Assessment methods used for AfL are usually non-standardized and therefore students' learning outcomes are described qualitatively in most situations. Because AfL focuses on the quality of the learning process, the form of the assessment is as important as the content of the assessment. For the assessment of deep learning, the use of various assessment methods is essential (Harlen \& Gardner, 2010). Instruments used in DT can be either standardized or adaptive, with the latter referring to computerized tests in which the selection and sequencing of items depends on the responses given by the student. The testing procedure of the instrument and the method of scoring should be based on measurement theories designed for DT (e.g., Leighton \& Gierl, 2007b; Rupp et al., 2010). Additionally, theories about students' cognitive processes are required to formulate hypotheses about the nature of students' learning outcomes. Because of the complexity of diagnostic measurement models, it is preferred that quantitative results of DT are reported in a readily usable format that contains an easy to understand qualitative description of the student's learning needs.

Furthermore, for each approach the requirements with regard to the quality of the data depend on the stakes of the decision: the need for objective and reliable data increases when the stakes become higher (Harlen, 2010). An example of a high-stakes decision is a pass/fail decision that is used to decide which students receive a diploma and which students will not. Even though it is desirable to use objective and reliable data for daily decisions, this is not always feasible because those data are often gathered with standardized (national) tests.

The time between the test moment and receiving reportage on the results of those tests often exceeds the time in which those decisions have to be made. Therefore, if the feedback loops are small, and the stakes are low, using various in-class assessment methods will usually suffice (Harlen \& James, 1997). Additionally, standardized tests are likely to be more focused on specific learning goals than other assessment methods, and for that reason are unable to fully cover the diverse meanings of students' learning outcomes within school curricula. 


\section{Characteristics of the Assessment Process}

The processes of both DDDM and DT are cyclic, systematic, and formal. However, the stakes that are associated with DDDM are usually higher than those associated with DT. The process of AfL is non-cyclic and non-systematic, because the gathering of data is as a result of student-teacher interactions in daily practice rather than of a planned and separate measurement moment. Therefore, students will be likely to perceive the process of AfL as informal.

\section{The Power Differentials between Agents}

a. Who provides feedback?

For DDDM, the feedback can be provided by an external party that develops standardized tests. When this is the case, the decision most likely has a high-stakes character. Additionally, in DDDM, feedback might also be provided by agents within the school. In both situations, the teacher is responsible for using this feedback within the classroom and for feeding these results back to the students. For AfL, feedback is only provided by agents within the school, such as a teacher, a peer, or a computer. In DT, the feedback is provided by the computer, either directly to the student, or indirectly via the teacher.

\section{b. Who determines what has to be learned?}

The learning outcomes assessed in DDDM are the learning objectives that are mandated by external parties like the government. In AfL, national learning objectives serve as a broader framework of learning goals, but do not determine what has to be learned on a day-to-day basis. Instead, the students and their teacher(s) decide upon the daily learning intentions. Although DT is concerned with the learning needs of individual students, what has to be learned is determined by the agents outside the school who developed the diagnostic test. Specifically, the theories about students' cognitive processes, common errors, and misconceptions determine what has to be learned in DT.

\section{Types of Intervention}

In the section about Characteristic 6, the process of DDDM is described as retroactive, meaning that the interventions that follow aim to remediate signaled learning difficulties. Feedback in DDDM has a quantitative nature and can be used to inform decisions on all educational levels. AfL is primarily interactive because interventions implemented in AfL, such as qualitative feedback, are part of the daily classroom discourse (Stobart, 2008). 
Specifically, the interventions in AfL are the result of the interaction between the learner and the learning environment (e.g., teacher, peers, and materials). When the quality of the interactions between the learner and the learning environment is optimal, AfL is also characterized as proactive because in that case the development of learning difficulties is highly improbable. DT is primarily retroactive; interventions are mostly used for the remediation of learning problems. For example, this is the case when students are classified and/or selected. However, as described above, DT becomes interactive when the diagnostic tests provide feedback to the student during the measurement moment. Additionally, DT can result in proactive interventions when it is used at the beginning of a teaching period to, for example, measure students' preconceptions so that the development of learning difficulties can be prevented.

\section{Learning Theory}

Initially, the improvement of learning outcomes was based on neo-behaviorist ideas, which encompass student learning in terms of learning sub-skills spread out over fixed periods of time within each school year (e.g., six weeks). After that period students' abilities are assessed, usually with a paper-and-pencil test (Stobart, 2008). As a consequence of this cyclic process, the formative function of those assessments is purely retroactive. As is implied by previously described characteristics, DDDM is based on these theoretical principles. However, critics of the neo-behaviorist principles argued that students' reasoning styles should be recognized as an indicator of their ability, which is known as the constructivist learning theory. In this theory, it is stated that learners actively construct their knowledge. This theory prescribes that assessments should not only focus on behavioral aspects of learning, but also on students' reasoning (Verhofstadt-Denève, 2003).

The theory on which AfL is based takes constructivism a step further by stating that knowledge is constructed through interactions with others (Stobart, 2008). This is called social constructivism. As described in the section about DT, DT is concerned with measuring the student's ZPD. The ZPD is an element within social constructivism; learning through interactions with others means learning with the minimal help of others. Thus, both AfL and DT are based on principles of social constructivism. 
Table 3 Results of the Comparison of the Three Assessment Approaches Described by Characteristics

\begin{tabular}{|c|c|c|c|}
\hline Assessment Characteristic & Data-Driven Decision Making & Assessment for Learning & Diagnostic Testing \\
\hline $\begin{array}{l}\text { 1. The object of the } \\
\text { assessment. }\end{array}$ & Individuals, classrooms, schools & Individuals and classrooms & Individuals. \\
\hline $\begin{array}{l}\text { 2. The object of decision } \\
\text { making. }\end{array}$ & $\begin{array}{l}\text { Individuals, subgroups within a } \\
\text { classroom, whole classrooms, multiple } \\
\text { classrooms, within a school, and across } \\
\text { schools. }\end{array}$ & $\begin{array}{l}\text { Individuals, subgroups within a } \\
\text { classroom, and whole classrooms. }\end{array}$ & Individuals. \\
\hline $\begin{array}{l}\text { 3. The time point in which } \\
\text { decisions need to be } \\
\text { made. } \\
\text { a. Timing of the } \\
\text { decision. } \\
\text { b. Frequency of the } \\
\text { decision. } \\
\text { c. Timing of the } \\
\text { feedback. }\end{array}$ & $\begin{array}{l}\text { a. Depending on the level at which a } \\
\text { decision has to be made this can } \\
\text { vary from immediately to a couple } \\
\text { of years. } \\
\text { b. Varies from daily to once every } \\
\text { couple of years, depending on the } \\
\text { stakes of the decision. } \\
\text { c. Data-feedback at the school and } \\
\text { classroom level, usually delayed. }\end{array}$ & $\begin{array}{l}\text { a. During the learning process. } \\
\text { b. When teaching, teachers } \\
\text { continuously need to decide upon } \\
\text { their next step. For these decisions } \\
\text { they use assessment information } \\
\text { that is available at that moment. } \\
\text { c. Continuously provided according } \\
\text { to the needs of the learners. }\end{array}$ & $\begin{array}{l}\text { a. Depending on the needs of the } \\
\text { learner(s). } \\
\text { b. Depending on the needs of the } \\
\text { learner(s). } \\
\text { c. Preferably right after the diagnosis } \\
\text { and advice on the intervention is } \\
\text { given. }\end{array}$ \\
\hline $\begin{array}{l}\text { 4. The objective of the } \\
\text { assessment. }\end{array}$ & $\begin{array}{l}\text { Determining and monitoring whether } \\
\text { learning goals have been achieved, and } \\
\text { investigating whether changes to the } \\
\text { learning environment are necessary. }\end{array}$ & $\begin{array}{l}\text { Improving the quality of the learning } \\
\text { process and thereby establishing } \\
\text { higher learner autonomy, and higher } \\
\text { learning outcomes. }\end{array}$ & $\begin{array}{l}\text { Assessment of processing activities, } \\
\text { and identifying preconceptions, } \\
\text { misconceptions, bugs, and problem- } \\
\text { solving strategies. }\end{array}$ \\
\hline $\begin{array}{l}\text { 5. The assessment methods } \\
\text { (instruments). }\end{array}$ & $\begin{array}{l}\text { - Standardized tests; } \\
\text { - quantitative results; and } \\
\text { - form follows content; larger focus on } \\
\text { what has to be learned than how it is } \\
\text { learned. }\end{array}$ & $\begin{array}{l}\text { - Non-standardized; } \\
\text { - mainly qualitative results; and } \\
\text { - the content and form of assessment } \\
\text { that are chosen are directly related to } \\
\text { how successful learning is } \\
\text { operationalized in terms of subject } \\
\text { knowledge and understanding, as } \\
\text { well as in terms of self-regulation } \\
\text { skills (Stobart, 2008). }\end{array}$ & $\begin{array}{l}\text { - Standardized or adaptive tests; } \\
\text { - quantitative results explained in } \\
\text { qualitative student reports; and } \\
\text { - the content and form are theory- } \\
\text { driven, because based on the } \\
\text { outcomes inferences have to be made } \\
\text { about why a student has achieved } \\
\text { those learning outcomes. }\end{array}$ \\
\hline
\end{tabular}


Table 3 (continued) Results of the Comparison of the Three Assessment Approaches Described by Characteristics

\begin{tabular}{|c|c|c|c|}
\hline Assessment Characteristic & Data-Driven Decision Making & Assessment for Learning & Diagnostic Testing \\
\hline $\begin{array}{l}\text { 6. Characteristics of the } \\
\text { assessment process. }\end{array}$ & $\begin{array}{ll}- & \text { Cyclic; } \\
- & \text { systematic; and } \\
- & \text { formal. }\end{array}$ & $\begin{array}{ll}- & \text { Non-cyclic (continuous); } \\
- & \text { non-systematic; and } \\
- & \text { informal. }\end{array}$ & $\begin{array}{ll}\text { - } & \text { Cyclic; } \\
\text { - } & \text { systematic; and } \\
- & \text { formal. }\end{array}$ \\
\hline $\begin{array}{l}\text { 7. The power differentials } \\
\text { between agents. } \\
\text { a. Who provides } \\
\text { feedback. } \\
\text { b. Who determines } \\
\text { what has to be } \\
\text { learned. }\end{array}$ & $\begin{array}{l}\text { a. Depending on the stakes of the } \\
\text { test, feedback on student results is } \\
\text { provided by external or internal } \\
\text { parties. The teacher is responsible } \\
\text { for feeding back these results into } \\
\text { the classroom. } \\
\text { b. External party (i.e., the } \\
\text { government). }\end{array}$ & $\begin{array}{l}\text { a. Peer, teacher, student(s), or } \\
\text { computer. } \\
\text { b. The teacher and the student decide } \\
\text { upon learning intentions, based on } \\
\text { students' learning needs, within a } \\
\text { framework of broader learning } \\
\text { goals. }\end{array}$ & $\begin{array}{l}\text { a. Depending on the assessment } \\
\text { method used, feedback is provided } \\
\text { by the teacher or the computer. } \\
\text { b. The teacher and/or the student } \\
\text { decide upon the student's personal } \\
\text { learning goals. }\end{array}$ \\
\hline 8. Types of intervention. & $\begin{array}{l}\text { - } \quad \text { Retroactive; } \\
\text { educational levels (the level at } \\
\text { which the decision is taken affects } \\
\text { the type of intervention); and } \\
\text { - quantitative feedback. }\end{array}$ & $\begin{array}{l}\text { - } \quad \text { Interactive; } \\
\text { - } \quad \text { adaptations to teaching; } \\
\text { adaptations to the learning } \\
\text { environment on single classroom } \\
\text { and on student level; and } \\
\text { - } \quad \text { qualitative feedback. }\end{array}$ & $\begin{array}{ll}- & \text { Proactive (prevention); } \\
- & \text { retroactive (remediation); } \\
- & \text { classification (also for } \\
& \text { differentiated teaching); } \\
- & \text { selection; and } \\
- & \text { quantitative and qualitative } \\
& \text { feedback. }\end{array}$ \\
\hline 9. Learning theory. & - $\quad$ Neo-behaviorism (Stobart, 2008) & $\begin{array}{l}\text { - Social constructivism (Stobart, } \\
\text { 2008). }\end{array}$ & $\begin{array}{ll}\text { - } & \text { Social constructivism; } \\
\text { - } & \text { cognitive developmental } \\
\text { psychology; and } \\
\text { - } \quad \text { information processing theories. }\end{array}$ \\
\hline
\end{tabular}




\section{Discussion}

The goal of this study was to investigate the extent to which DDDM, AfL, and DT can be shaped into an integrative formative approach towards assessment. The three approaches to assessment claim to be beneficial with regard to student learning. However, the literature has pointed out that different meanings are ascribed to student learning within these approaches; on the one hand student learning is defined as achieving learning goals, and on the other hand it is referred to as the quality of the learning process (see James \& Brown, 2005, for more possible meanings of learning outcomes). If the three approaches can be combined into an integrative formative approach towards assessment, this could maximize student learning, in terms of both the process and the outcomes of learning at all levels of education. The three approaches were compared on nine characteristics of assessment.

The results suggest that the approaches could complement each other with respect to the objects of assessments, the objects of the decisions, the time point in which decisions need to be made, the assessment methods, and characteristics of the assessment process. With respect to the objects of assessments and the objects of the decisions, DDDM comprises all educational levels, whereas AfL comprises the classroom level and individuals. DT only concerns individuals. If these approaches are to be integrated, this would mean that DDDM would serve as a more overarching approach concerning evaluation and monitoring. Furthermore, in our view, DDDM should be concerned with high-stakes decisions, whereas AfL should concern the daily practice where decisions are made on a continuous basis. DT should be used when needed to gather indepth information about student learning. The assessment methods differ widely between the three approaches; however, we believe that they should be present simultaneously. Namely, when

all three approaches are combined this will lead to a complete picture of both students' learning processes and learning outcomes. Also, the characteristics of the assessment process differ, but can be complementary. Since DDDM is highly systematic and cyclic, it can be used to maintain and improve the quality of education. Useful tools for this purpose for example are student monitoring systems, in which students' learning outcomes are assessed using standardized tests once or twice a year. When the results of these monitoring actions suggest that learning goals are not being met, it has to be decided how the learning environment could be changed to improve students' learning outcomes. 
On a day-to-day basis in the classroom, however, AfL can be a very powerful approach. Because of its flexible and responsive character, the learning needs of students can best be attended to. DT can be used in a flexible way, when there is a need for more in-depth information about student learning or particular learning needs.

The objectives of the assessments and the interventions in the three approaches were found to be somewhat contradictory. More specifically, DDDM has a retroactive character, whereas AfL and DT are more proactive, interactive, as well as retroactive. We believe that when the three approaches are implemented simultaneously, the need for retroactive measurers will decline, because learning difficulties will be resolved immediately. Therefore, we argue that when sufficient autonomy is granted to the students and teachers, the three approaches could actually support each other. The power differentials between agents appeared to differ between the three approaches as well. The results suggest that high power differentials between agents, as is the case in DDDM, will give students and teachers less opportunities to take responsibility for the quality of the learning process, a necessary condition for AfL. DDDM is more distant from teachers and students, because it mainly takes place outside the classroom, and therefore the power differentials are high. Thus, power differentials between agents in AfL, and to a lesser extent in DT, are much smaller than in DDDM because teachers decide on the learning activities that take place in their classroom. We believe that when the power differentials at the classroom level are low, this will offer the most optimal learning climate in which feedback can be used to its full potential. However, low power differentials in AfL require that teachers receive autonomy from school principles to design their practice. Moreover, the learning theory on which DDDM is based, known as neo-behaviorism, has in most educational reforms been replaced with the (social) constructivist view of learning (Stobart, 2008). Because our comparison shows that both AfL and DT are based on social constructivism and this appears to be the theory that currently dominates education, we believe it would be best if this theory was also used for DDDM. Socialconstructivism can be used in DDDM because it does not exclude neo-behaviorist principles. Moreover, it supplements those principles by addressing the cognitive and social components of learning. Pedagogical-didactical principles in neo-behaviorism focus on conditioning, meaning students internalize how to solve tasks by repeatedly performing the same tasks (VerhofstadtDenève, et al., 2003). 
A DDDM approach that is more social-constructivist oriented includes learning activities that are active (not merely reproducing and repeating the same tasks) and include frequent interactions with peers and the teacher. Furthermore, concerning the ecological validity of assessment methods used within the three approaches it is important to use methods that are similar to the wide variety of learning tasks used in social-constructivist learning environments. However, assessment methods often used in DDDM are standardized tests with a fixed question format. Thus, a DDDM approach based on a social-constructivist view of learning acknowledges the complexity of learning by including various assessment methods.

Furthermore, DT complements DDDM and AfL because it offers teachers a non-laborintensive way of systematically collecting detailed data on a student's learning needs. Moreover, DT has a lower risk of misinterpretation of data than DDDM, because the quantitative data are described in wording that is easy to understand and can be directly used in practice. This is in contrast to DDDM, where the data has to be interpreted. For AfL, there is a low risk of making unjustified decisions as a result of misinterpretations, because misinterpretations will be directly revealed through teacher-student interactions, and can be contingently restored.

Nevertheless, the three approaches have some characteristics that might limit their formative potentials. In DDDM, for example, monitoring students could easily transform into frequent administration of mini summative tests (Harlen \& James. 1997; Stobart, 2008), which would unnecessarily raise the stakes for both students and teachers. Even more important, data-feedback from monitoring activities is most likely aggregated quantitative data that cannot easily be used to enhance the learning of individual students, because those data do provide sufficient detail about individual students. A possible pitfall of AfL is that teachers require extensive knowledge of the assessment domain to be able to, for example, ask questions that will promote students' learning during classroom discourse (Bennett, 2011; Moyer \& Milewicz, 2002). In the section about DT, it was explained that for the development of diagnostic tests, theories are required to make inferences about students' reasoning during the tests. In AfL, teachers need to develop similar theories to make distinctions between students' 'slips' and errors (Bennett, 2011). Besides formal teacher training programs, the use of DT might enhance teachers' knowledge about students' thinking with regard to a specific domain. Subsequently, the necessity of theories about how students construct knowledge and about their thinking within both AfL and DT becomes clear from our comparison of the theoretical underpinnings of both approaches. 
Finally, a pitfall of DT is that is only advises on the intervention, selection, and classification of students and therefore a formative use is not guaranteed.

A limitation of the current study is that the three approaches were only compared on their theoretical principles as described in educational literature. However, the definitions of the approaches are not uniformly described in the literature. For the sake of comparing the three approaches, interpretation of the meaning of these approaches was unavoidable. Further research should investigate how this integrative formative approach could be implemented in practice. Currently, in schools in British Columbia, Canada DDDM and AfL are the focus of their accountability framework (Ministry of Education, British Columbia, Canada, 2002). They define accountability in terms of the achievements of each student, and take into account the diversity of students within different school districts by adapting expectations based on DDDM and AfL. In addition to our comparison of DDDM, AfL, and DT, it would be interesting to further study initiatives such as these.

\section{References}

Assessment Reform Group (1999). Assessment for Learning: Beyond the black box. Cambridge University.

Assessment Reform Group (2002). Assessment is for Learning: 10 principles. Research-based principles to guide classroom practice. Retrieved on April 13th 2012 from http://assessmentreformgroup.files.wordpress.com/2012/01/10principles_english.pdf

Bennett, R. E. (2011). Formative assessment: A critical review. Assessment in Education: Principles, Policy \& Practice, 18, 5-25. doi:10.1080/0969594X.2010.513678

Black, P. \& Wiliam, D. (1998). Inside the black box. Raising standards through classroom assessment. Phi Delta Kappan, 80(2), 139-148.

Briggs, D. C. \& Alonzo, A. C. (2009). The psychometric modeling of ordered multiple choice item responses for diagnostic assessment with a learning progression. Learning Progressions in Science (LeaPS). Iowa City, IA. Retrieved on November 10th, 2011 from $\underline{\text { http://education.msu.edu/projects/leaps/proceedings/Briggs.pdf }}$

Carless, D. (2007). Conceptualizing pre-emptive formative assessment. Assessment in Education, 14(2), 171-184. doi:10.1080/09695940701478412

Davenport, T. H. \& Prusak, L. (1998). Working knowledge: How organizations manage what they know. Boston: Harvard Business School Press. 
De Bruyn, E. E. J., Ruijssenaars, A. J. J. M., Pameijer, N. K., \& Van Aarle, E. J. M. (2003). De diagnostische cyclus. Een praktijkleer [The diagnostic cycle. Practical guidelines]. Leuven, Begium: Acco.

Hargreaves, E. (2005). Assessment for Learning? Thinking outside the (black) box. Cambridge Journal of Education, 35(2), 213-224. doi:10.1080/03057640500146880

Harlen, W. (2007). The quality of learning: Assessment alternatives for primary education. Interim Reports. Cambridge: University of Cambridge. Retrieved on August 10th, 2011 from www.primaryreview.org.uk

Harlen, W. (2010). What is quality teacher assessment? In J. Gardner, W. Harlen, L. Hayward, and G. Stobart (Eds.), Developing teacher assessment (pp. 29-52). Maidenhead, England: Open University Press.

Harlen, W. \& Gardner, J. (2010). Assessment to support learning. In J. Gardner, W. Harlen, L. Hayward, and G. Stobart (Eds.), Developing teacher assessment (pp. 15-28). Maidenhead, England: Open University Press.

Harlen, W. \& James, M. (1997). Assessment and learning: Differences between formative and summative assessment. Assessment in Education: Principles, Policy, \& Practice, 4, 365379. doi:10.1080/0969594970040304

James, M. \& Brown, S. (2005). Grasping the TLRP nettle: preliminary analysis and some enduring issues surrounding the improvement of learning outcomes. The Curriculum Journal, 16, 7-30. doi:10.1080/0958517042000336782

Johnson, M. \& Burdett, N. (2010). Intention, interpretation and implementation: Some paradoxes of assessment for learning across educational contexts. Research in Comparative and International Education, 5, 122-130. doi:10.2304/rcie.2010.5.2.122

Keeley, P. \& Tobey, C. R. (2011). Mathematics formative assessment. Thousand Oaks, CA: Corwin.

Kievit, Th., Tak, J. A., \& Bosch, J. D. (Eds.), (2002). Handboek psychodiagnostiek voor de hulpverlening aan kinderen ( $6^{\text {th }}$ ed.) [Handbook psychodiagnostics in healthcare for children]. Utrecht, The Netherlands: De Tijdstroom.

Klenowski, V. (2009). Assessment for Learning revisited: An Asia-Pacific perspective. Assessment in Education: Principles, Policy, \& Practice, 16, 263-268. doi:10.1080/09695940903319646 
Ledoux, G., Blok, H., Boogaard, M., \& Krüger, M. (2009). Opbrengstgericht werken. Over waarde van meetgestuurd onderwijs [Data-driven decision making. About the value of measurement oriented education]. SCO-Rapport 812. Amsterdam: SCO-Kohnstamm Instituut.

Leighton, J. P. \& Gierl, M. J. (2007a). Defining and evaluating models of cognition used in educational measurement to make inferences about examinees' thinking processes. Educational Measurement: Issues and Practice, 26, 3-16. doi:10.1111/j.17453992.2007.00090.x

Leighton, J. P. \& Gierl, M. J. (Eds.) (2007b). Cognitive diagnostic assessment for education. Theory and applications. New York: Cambridge University Press.

Light, D., Wexler, D., \& Heinze, J. (2004). How practitioners interpret and link data to instruction: Research findings on New York City schools' implementation of the grow network. Paper presented at the annual meeting of the American Educational Research Association, San Diego, CA.

Locke, E.A., \& G. Latham (2002). Building a practically useful theory of goal setting and task motivation. The American Psychologist, 57(9), 705-717. doi:10.1037/0003-066X.57.9.705

Mandinach, E. B., Honey, M., \& Light, D. (April, 2006). A theoretical framework for data-driven decision making. Paper presented at the annual meeting of the American Educational Research Association, San Francisco.

Meijer, J., Ledoux, G., \& Elshof, D. P. (2011). Gebruikersvriendelijke leerlingvolgsystemen in het primair onderwijs [User friendly student monitoring systems in primary education]. Rapport 849, ISBN 90-6813-914-3. Amsterdam: Kohnstamm Instituut.

Ministry of Education, British Columbia, Canada (2002). Accountability Framework. Retrieved on April $13^{\text {th }}, 2012$ from http://www.bced.gov.bc.ca/policy/policies/accountability_framework.htm

Moyer, P. S. \& Milewicz, E. (2002). Learning to question: Categories of questioning used by preservice teachers during diagnostic mathematics interviews. Journal of Mathematics Teacher Education, 5, 293-315. doi:10.1023/A:1021251912775

Popham, W. J., Cruse, K. L., Rankin, S. C., Sandifer, P. D., \& Williams, R. L. (1985). Measurement-driven instruction. Phi Delta Kappan, 66, 628-634. 
Rupp, A. A., Templin, J., \& Henson, R. A. (2010). Diagnostic measurement. Theory, methods, and applications. New York: The Guilford Press.

Sanders, P. (2011). Het doel van toetsen [The purpose of tests]. In P. Sanders (Ed.), Toetsen op school [Testing at school] (pp. 9-20). Arnhem: Cito.

Schildkamp, K. \& Kuiper, W. (2010). Data-informed curriculum reform: Which data, what purposes, and promoting and hindering factors. Teaching and Teacher Education, 26, 482-496. doi:10.1016/j.tate.2009.06.007

Scriven, M. (1967). The Methodology of Evaluation. Washington, DC: American Educational Research Association.

Shepard, L. A. (2005, October). Formative assessment: Caveat emptor. Paper presented at the ETS Invitational Conference, The Future of Assessment: Shaping Teaching and Learning, New York.

Slavin, R. E. (2002). Evidence-based education policies: Transforming educational practice and research. Educational Researcher, 21(7), 15-21. doi:10.3102/0013189X031007015

Slavin, R. E. (2003). A reader's guide to scientifically based research. Educational Leadership, 60(5), 12-16.

Stobart, G. (2008). Testing times: The uses and abuses of assessment. London: Routledge.

Swan, G. \& Mazur, J. (2011). Examining data driven decision making via formative assessment: A confluence of technology, data interpretation heuristics and curricular policy. Contemporary Issues in Technology and Teacher Education, 11(2), 205-222.

Third Assessment for Learning Conference. (2009). Third International Conference on Assessment for for Learning, March 15-20 in Dunedin, New Zealand.

Turner, M., VanderHeide, K., \& Fynewever, H. (2011). Motivations for and barriers to the implementation of diagnostic assessment practices - a case study. Chemistry Education Research and Practice, 12, 142-157. doi:10.1039/C1RP90019F

Verhofstadt-Denève, L., Van Geert, P., \& Vyt, A. (2003). Handboek ontwikkelingspsychologie. Grondslagen en theorieën [Handbook developmental psychology. Principles and theories]. Houten: Bohn Stafleu Van Loghum.

Wayman, J. C. (2005). Involving teachers in data-driven decision making: Using computer data systems to support teacher inquiry and reflection. Journal of Education for Students Placed at Risk, 10, 295-308. doi:10.1207/s15327671espr1003_5 
Wayman, J. C., Cho, V., \& Johnston, M. T. (2007). The data-informed district: A district-wide evaluation of data use in the Natrona Country School District. Austin: The University of Texas.

Wiliam, D. (2011). What is Assessment for Learning? Studies in Educational Evaluation, 37, 314. doi:10.106/j.stueduc.2011.03.001

Wohlstetter, P., Datnow, A., \& Park, V. (2008). Creating a system for data-driven decisionmaking: applying the principal-agent framework. School Effectiveness and School Improvement, 19(3), 239-259. doi:10.1080/09243450802246376

Young, V. M., \& Kim, D. H. (2010). Using assessments for instructional improvement: A literature review. Educational Policy Analysis Archives, 18(19) 
